\title{
Molecular Cursor Caliper: A Fluorescent Sensor for Dicarboxylate Dianions
}

Wei Chen, ${ }^{\dagger, \downarrow}{ }^{+}$Chenxing Guo, ${ }^{\dagger, \perp}$ Qing He ${ }^{\dagger}$ Xiaodong Chi, ${ }^{\dagger}$ Vincent M.

Lynch, ${ }^{\dagger}$ Zhiyun Zhang, ${ }^{\star}$ Jianhua Su, ${ }^{\star}$ He Tian, ${ }^{*}{ }^{\star}$ and Jonathan L. Sessler ${ }^{*}{ }^{\dagger}$

${ }^{\dagger}$ Department of Chemistry, The University of Texas at Austin, 105 E. 24th Street, Stop A5300, Austin, TX 78712-1224, USA

Key Laboratory for Advanced Materials and Joint International Research Laboratory of Precision Chemistry and Molecular Engineering, Feringa Nobel Prize Scientist Joint Research Center, East China University of Science and Technology, Shanghai 200237, China

${ }^{\perp}$ W. C. and C. G. contributed equally

\section{Table of Contents}

1. General information

2. Synthesis and characterization.

3. UV-vis and fluorescent spectral response to linear saturated dicarboxylates $\left(\mathrm{Cn}^{2-}\right)$. .56

4. Determination of the binding stoichiometry S13

5. Fluorescent response to the three phthalate isomers .$S 15$

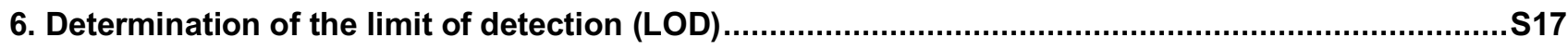

7. Computational studies .521

8. Crystal structures of DPAC-bis/monoCHO and DPAC-bisC4P .546

9. X-ray experimental .547

10. ${ }^{1} \mathrm{H}$ NMR, ${ }^{13} \mathrm{C}$ NMR, and HRMS spectra. .549

11. References S55 


\section{General information}

Synthesis and characterization. 9,14-Diphenyl-9,14-dihydrodibenzo[a,c]phenazine (DPAC) was synthesized according to a previous literature report. ${ }^{\mathrm{S} 1}$ Other reagents were purchased from commercial suppliers (e.g., Fisher, Aldrich, TCI, and Acros, etc.) and used without further purification. All deuterated solvents were purchased from Cambridge Isotope Laboratories and Aldrich Chemical Co. ${ }^{1} \mathrm{H}$ and ${ }^{13} \mathrm{C}$ NMR spectra were recorded on a Varian Mercury 400 instrument using tetramethylsilane (TMS) as the internal standard, operating at 400 and $100 \mathrm{MHz}$, respectively. Chemical shifts are recorded in units of $\delta(\mathrm{ppm})$ and referenced to the residual solvent. Generally, deuterated sulfoxide (DMSO- $d_{6}$ ) was used as the solvent for the ${ }^{1} \mathrm{H}$ NMR spectral studies because its solvent residual peaks do not overlap with the compound peaks. However, due to the low solubility of some compounds in DMSO- $d_{6}$, deuterated chloroform $\left(\mathrm{CDCl}_{3}\right)$ was chosen as an alternative solvent for recording the ${ }^{13} \mathrm{C}$ NMR spectra. High-resolution mass spectrometric (HRMS) analyses were made using a VG AutoSpec apparatus. X-ray crystallographic analyses were carried out on an Agilent Technologies SuperNova Dual Source diffractometer using a $\mu$-focus $\mathrm{Cu}$ K $\alpha$ radiation source $(\lambda=1.5418 \AA)$ with collimating mirror monochromators or on a Nonius Kappa CCD diffractometer using a Bruker AXS Apex II detector and a graphite monochromator with Mo Ka radiation $(\lambda=0.71073 \AA)$. The CIF files for the crystallographic data have been deposited in the Cambridge Crystallographic Data Centre, and the CCDC numbers are shown in Table S2 (cf. http://www.ccdc.cam.ac.uk)

Spectroscopic measurements. UV-vis spectra were recorded at room temperature using a Varian Cary 5000 UV-vis-NIR spectrophotometer. Steady-state emission spectra were acquired on an Agilent Cary Eclipse fluorescence spectrophotometer. A cuvette length of $10 \mathrm{~mm}$ was used for all UV-vis and fluorescence spectral studies.

Binding Studies. All fluorescence spectroscopic titrations were carried out in commercially available Fisher Scientific acetonitrile $\left(\mathrm{CH}_{3} \mathrm{CN}\right)$. All anions were studied in the form of their tetrabutylammonium salts. The receptor concentration was kept constant over the course of the experiment. The following equation was used to determine the binding affinities in the case of 1:1 binding at any given wavelength:

$$
y=\frac{I-I_{0}}{I_{\mathrm{F}}-I_{0}}=\frac{1+a K+x K-\sqrt{-4 a x K^{2}+(-1-a K-x K)^{2}}}{2 K} \times E
$$

where $\left(I-I_{0}\right) /\left(I_{\mathrm{F}}-I_{0}\right)$ (normalized change in emission intensity) is the $y$ variable; $x$ is the concentration of the added guest; $a$ is the initial concentration of the receptor (kept constant over the course of titration); $E$ is a coefficient (the derivation of this value from basic photophysical constants is omitted for clarity); $K_{\mathrm{a}}$ is the binding affinity of the receptor for the guest in question.

OriginPro $2018 \mathrm{~b}$ was used to fit data in a non-linear fashion in accord with the above equation. Iterations (choosing a fixed $a$ value and changing $\Delta \varepsilon$ and $K$ ) were performed until an acceptable level of convergence was reached (chi-square tolerance value $\leq 1 \times 10^{-9}$ ).

In the case of 1:2 binding (i.e., DPAC-bisC4P and $\mathbf{C n}^{2-}$, where $n=2$ or 4), the fitting was performed using OriginPro according to a protocol we developed and reported previously. ${ }^{\mathrm{S} 2}$ In addition, the original spectroscopic titration data for $\mathbf{C 2}^{2-}$ and $\mathbf{C 4}^{2-}$ were also fitted using the BindFit web applet available at www.supramolecular.org. As detailed in the main text, near-concordant values for the binding constants were obtained using these two fitting approaches. 
Computational methods. The ground-state structures for all DPAC-bisC4P $\supset D^{2-}$ complexes investigated in this study were optimized using a semi-empirical method in the gas phase at PM6 level. The first excited-state structures for DPAC-bisC4P and DPAC-bisC4P $\supset \mathbf{C 4}^{2-}$ were optimized using a time-dependent semi-empirical method in the gas phase at the PM6 level. All theoretical calculations were performed using Spartan 16 or Gaussian $16 .^{\mathrm{S} 3}$

\section{Synthesis and characterization}

Scheme S1. Synthesis of compounds DPAC-bisCHO and DPAC-monoCHO.

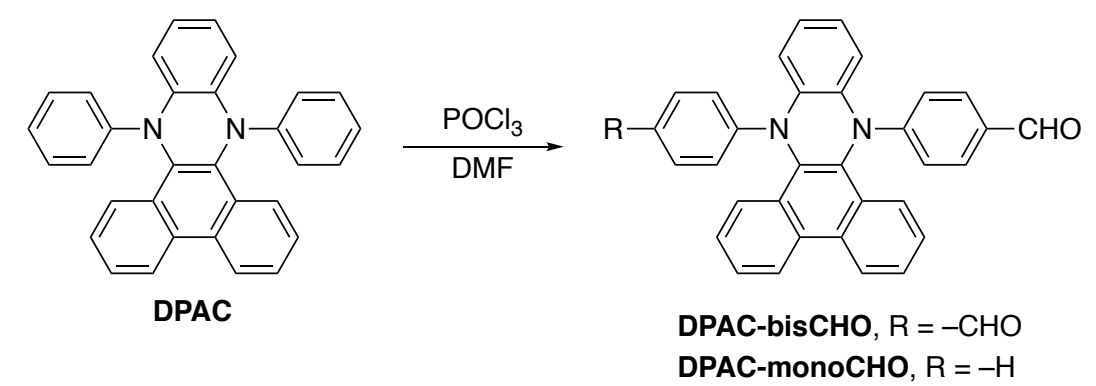

\section{General procedure for the synthesis of DPAC-bis/monoCHO.}

DPAC (1.0 g, $2.3 \mathrm{mmol})$ was dissolved in $20 \mathrm{~mL}$ of dry DMF in an oven-dried three-neck round-bottom flask. Phosphorus oxychloride, $(4.30 \mathrm{~mL}, 46.0 \mathrm{mmol})$ for the preparation of DPAC-bisCHO; $(2.15 \mathrm{~mL}, 23.0 \mathrm{mmol})$ in the case of DPAC-monoCHO), was added dropwise under a nitrogen atmosphere at $0{ }^{\circ} \mathrm{C}$. After stirring at $0{ }^{\circ} \mathrm{C}$ for $1 \mathrm{~h}$, the reaction mixture was heated to $70{ }^{\circ} \mathrm{C}$ and stirred overnight. The resulting solution was then allowed to cool to room temperature and poured into an ice-water mixture. The $\mathrm{pH}$ of the solution was adjusted to 7 by adding $1 \mathrm{M} \mathrm{NaOH}$. $\mathrm{A}$ precipitate formed during this process. The precipitate (crude product) was filtered off and purified by column chromatography over silica gel (eluent: petroleum ether/dichloromethane (DCM)) to give DPAC-bisCHO and DPAC-monoCHO in 30\% and 40\% yield, respectively.

4,4'-(Dibenzo[a,c]phenazine-9,14-diyl)dibenzaldehyde (DPAC-bisCHO): ${ }^{1} \mathrm{H}$ NMR (400 MHz, CDCl ${ }_{3}$ ): $\delta 9.69$ (s, $2 \mathrm{H}$ ), $8.81(\mathrm{~d}, 2 \mathrm{H}, J=8.3 \mathrm{~Hz}), 8.05(\mathrm{~d}, 2 \mathrm{H}, J=8.0 \mathrm{~Hz}), 7.83\left(\mathrm{dt}, 2 \mathrm{H}, J_{1}=7.3 \mathrm{~Hz}, J_{2}=3.7 \mathrm{~Hz}\right), 7.75\left(\mathrm{t}, 2 \mathrm{H}, J_{1}=7.7 \mathrm{~Hz}\right), 7.63(\mathrm{t}$, $2 \mathrm{H}, J=7.6 \mathrm{~Hz}), 7.52(\mathrm{~d}, 4 \mathrm{H}, J=8.9 \mathrm{~Hz}), 7.48\left(\mathrm{dd}, 2 \mathrm{H}, J_{1}=5.9 \mathrm{~Hz}, J_{2}=3.5 \mathrm{~Hz}\right), 6.98(\mathrm{~d}, 4 \mathrm{H}, J=8.9 \mathrm{~Hz}) .{ }^{13} \mathrm{C} \mathrm{NMR}(100$ $\mathrm{MHz}_{\mathrm{CDCl}}$ ): $\delta 190.6,151.4,143.7,137.9,131.3,130.2,129.3,128.5,128.1,127.6,127.5,126.6,124.4,123.5,114.5$. HRMS (ESI+) $m / z:[\mathrm{M}+\mathrm{Na}]^{+}$calcd for $\mathrm{C}_{34} \mathrm{H}_{22} \mathrm{~N}_{2} \mathrm{O}_{2} \mathrm{Na}, 513.1579$; found, 513.1578.

4-(14-Phenyldibenzo $[a, c] p h e n a z i n-9(14 H)$-yl)benzaldehyde (DPAC-monoCHO): ${ }^{1} \mathrm{H}$ NMR (400 MHz, DMSO- $\left.d_{6}\right): \delta$ $9.69(\mathrm{~s}, 1 \mathrm{H}), 8.99\left(\mathrm{dd}, J_{1}=8.3 \mathrm{~Hz}, J_{2}=3.3 \mathrm{~Hz}, 2 \mathrm{H}\right), 8.03\left(\mathrm{dd}, J_{l}=6.0 \mathrm{~Hz}, J_{2}=3.4 \mathrm{~Hz}, 1 \mathrm{H}\right), 7.99-7.94(\mathrm{~m}, 3 \mathrm{H})$, 7.80-7.68 (m, 3H), $7.63\left(\mathrm{dd}, J_{1}=12.4 \mathrm{~Hz}, J_{2}=8.2 \mathrm{~Hz}, 3 \mathrm{H}\right), 7.53-7.47(\mathrm{~m}, 2 \mathrm{H}), 7.11-7.00(\mathrm{~m}, 6 \mathrm{H}), 6.84(\mathrm{t}, J=6.9 \mathrm{~Hz}$, 1H). ${ }^{13} \mathrm{C} \mathrm{NMR}\left(100 \mathrm{MHz}, \mathrm{CDCl}_{3}\right): \delta$ 190.6, 152.2, 146.9, 145.3, 143.1, 139.0, 136.8, 131.2, 130.2, 129.8, 129.1, 128.9, $128.9,128.7,127.7,127.5,127.3,127.1,127.0,126.9,126.4,125.4,124.8,124.0,123.3,123.1,121.6,117.0,114.4$. HRMS (ESI+) $m / z:[\mathrm{M}+\mathrm{H}]^{+}$calcd for $\mathrm{C}_{33} \mathrm{H}_{23} \mathrm{~N}_{2} \mathrm{O}, 463.1810$; found, 463.1804 . 
Scheme S2. Synthesis of compounds DPAC-bisOH and DPAC-monoOH.

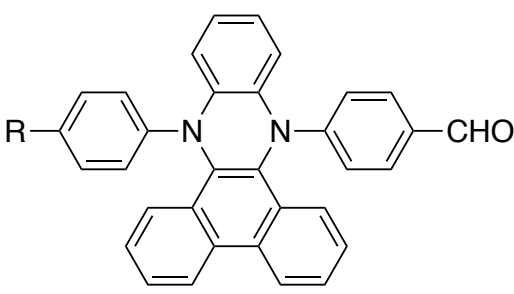

DPAC-bisCHO, $\mathrm{R}=-\mathrm{CHO}$ DPAC-monoCHO, $\mathrm{R}=-\mathrm{H}$

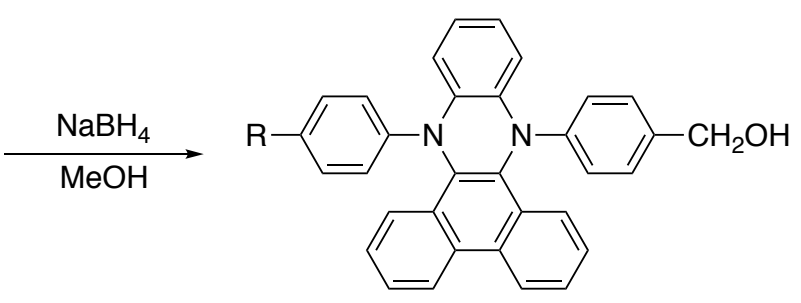

DPAC-bisOH, $\mathrm{R}=-\mathrm{CH}_{2} \mathrm{OH}$ DPAC-monoOH, $\mathrm{R}=-\mathrm{H}$

\section{General procedure for the synthesis of DPAC-bis/monoOH.}

DPAC-bisCHO (981 mg, $2.0 \mathrm{mmol}$ ) or DPAC-monoCHO (925 mg, $2.0 \mathrm{mmol}$ ) was dissolved in $30 \mathrm{~mL}$ of methanol. The resulting solution was cooled to $0{ }^{\circ} \mathrm{C}$ by means of ice-water bath. Sodium borohydride $(20 \mathrm{mmol})$ was then added portion-wise. After stirring overnight at room temperature, the reaction mixture was poured into an ice-water mixture. The resulting crude product, a beige precipitate, was filtered off and recrystallized from toluene. This afforded a white product in ca. 70\% yield in the case of both DPAC-bisOH and DPAC-monoOH.

(Dibenzo[a,c]phenazine-9,14-diylbis(4,1-phenylene))dimethanol (DPAC-bisOH): ${ }^{1} \mathrm{H}$ NMR (400 MHz, DMSO- $d_{6}$ ): $\delta$ $8.93(\mathrm{~d}, 2 \mathrm{H}, J=8.3 \mathrm{~Hz}), 7.94(\mathrm{~d}, 2 \mathrm{H}, J=7.3 \mathrm{~Hz}), 7.89$ (dd, 2H, $\left.J_{1}=5.9 \mathrm{~Hz}, J_{2}=3.5 \mathrm{~Hz}\right), 7.70(\mathrm{t}, 2 \mathrm{H}, J=7.0 \mathrm{~Hz})$, $7.62-7.57(\mathrm{~m}, 2 \mathrm{H}), 7.40\left(\mathrm{dd}, 2 \mathrm{H}, J_{1}=5.9 \mathrm{~Hz}, J_{2}=3.4 \mathrm{~Hz}\right), 7.06-6.98(\mathrm{~m}, 8 \mathrm{H}), 4.97(\mathrm{t}, 2 \mathrm{H}, J=5.7 \mathrm{~Hz}), 4.30(\mathrm{~d}, 4 \mathrm{H}, J=$ 5.7Hz). ${ }^{13} \mathrm{C}$ NMR (100 MHz, $\left.\mathrm{CDCl}_{3}\right): \delta 147.3,144.9,138.3,133.1,129.9,129.4,128.1,127.6,127.1,126.7,125.5$, 124.5, 123.1, 116.4, 65.0. HRMS (ESI+) $m / z$ : $[\mathrm{M}+\mathrm{Na}]^{+}$calcd for $\mathrm{C}_{34} \mathrm{H}_{26} \mathrm{~N}_{2} \mathrm{O}_{2} \mathrm{Na}$, 517.1892; found, 517.1896.

(4-(14-Phenyldibenzo[a,c]phenazin-9(14H)-yl)phenyl)methanol (DPAC-monoOH): ${ }^{1} \mathrm{H}$ NMR (DMSO- $d_{6}, 400 \mathrm{MHz}$ ): $\delta 8.88(\mathrm{~d}, 2 \mathrm{H}, J=8.3 \mathrm{~Hz}), 7.92\left(\mathrm{dd}, 2 \mathrm{H}, J_{1}=11.3 \mathrm{~Hz}, J_{2}=8.2 \mathrm{~Hz}\right), 7.85\left(\mathrm{ddd}, 2 \mathrm{H}, J_{1}=9.3 \mathrm{~Hz}, J_{2}=6.7 \mathrm{~Hz}, J_{3}=3.9 \mathrm{~Hz}\right)$, $7.69-7.63(\mathrm{~m}, 2 \mathrm{H}), 7.56\left(\mathrm{dt}, 2 \mathrm{H}, J_{1}=10.3 \mathrm{~Hz}, J_{2}=7.6 \mathrm{~Hz}\right), 7.39-7.35(\mathrm{~m}, 2 \mathrm{H}), 7.07-6.95(\mathrm{~m}, 8 \mathrm{H}), 6.79(\mathrm{t}, 1 \mathrm{H}, J=7.2$ $\mathrm{Hz}), 4.26(\mathrm{~d}, 2 \mathrm{H}, J=5.6 \mathrm{~Hz}) .{ }^{13} \mathrm{C}$ NMR (DMSO- $d_{6}, 100 \mathrm{MHz}$ ): $\delta$ 147.7, 146.3, 144.6, 144.0, 137.5, 137.1, 136.3, 129.9, $129.8,129.4,128.8,128.7,127.8,127.7,127.6,127.5,127.3,127.3,127.1,126.2,125.9,124.2,124.2,124.0,121.7$, 117.8, 117.0, 62.8. HRMS (ESI+) $m / z:[\mathrm{M}+\mathrm{H}]^{+}$calcd for $\mathrm{C}_{33} \mathrm{H}_{25} \mathrm{~N}_{2} \mathrm{O}, 465.1961$; found, 465.1955 .

Scheme S3. Synthesis of compounds DPAC-bisC4P and DPAC-monoC4P.

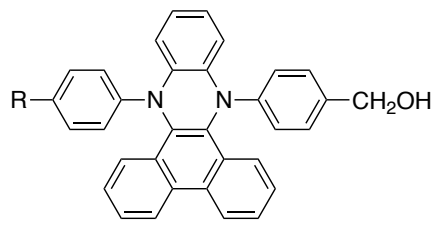

DPAC-bisOH, $\mathrm{R}=-\mathrm{CH}_{2} \mathrm{OH}$ DPAC-monoOH, $\mathrm{R}=-\mathrm{H}$

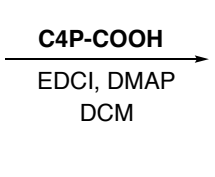

DCM
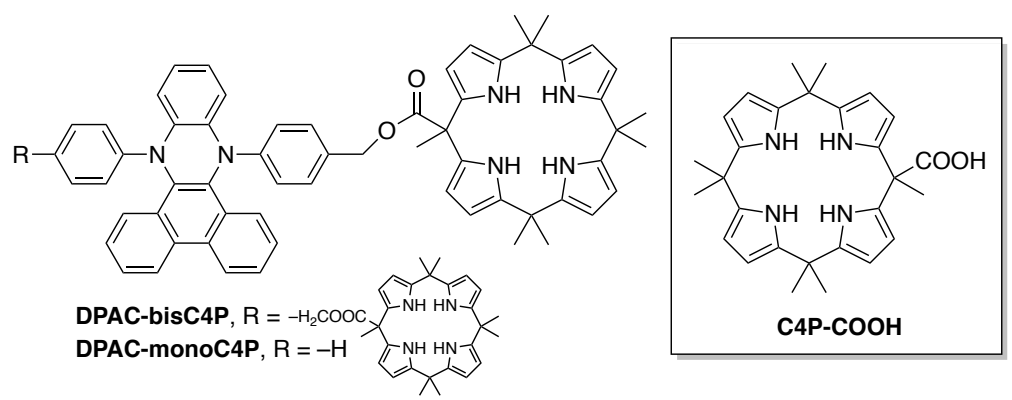

General procedure for synthesis of DPAC-bis/monoC4P.

Compound C4P-COOH was prepared according to a previous reported procedure. ${ }^{\mathrm{S4}}$ Compounds DPAC-bisOH (247 $\mathrm{mg}, 0.50 \mathrm{mmol}$ ) or DPAC-monoOH (232 mg, $0.50 \mathrm{mmol})$ and C4P-COOH (1.15 g, $2.5 \mathrm{mmol})$ were dissolved in $50 \mathrm{~mL}$ dichloromethane. 4-Dimethylaminopyridine (DMAP, $733 \mathrm{mg}, 6.0 \mathrm{mmol}$ ) was added to the solution under a nitrogen atmosphere. After cooling to $0{ }^{\circ} \mathrm{C}$, 1-ethyl-3-(3-dimethylaminopropyl)carbodiimide hydrochloride (EDCI, $776 \mathrm{mg}, 5.0$ mmol) was added. The resulting reaction mixture was stirred for $0.5 \mathrm{~h}$ at $0{ }^{\circ} \mathrm{C}$. It was then allowed to warm to room 
temperature. After stirring overnight, the reaction mixture was washed with water and extracted with DCM. The combined DCM extracts were dried over anhydrous magnesium sulfate. The resulting crude product was purified by column chromatography over silica gel (eluent: DCM) to afford the products in the form of white solids in $40 \%$ and $60 \%$ yield for DPAC-bisC4P and DPAC-monoC4P, respectively.

DPAC-bisC4P: ${ }^{1} \mathrm{H}$ NMR (400 MHz, DMSO- $\left.d_{6}\right): \delta 9.53(\mathrm{~s}, 4 \mathrm{H}), 9.30(\mathrm{~s}, 4 \mathrm{H}), 8.94(\mathrm{~d}, 2 \mathrm{H}, J=8.3 \mathrm{~Hz}), 7.88\left(\mathrm{dd}, 4 \mathrm{H}, J_{1}=\right.$ $\left.8.9 \mathrm{~Hz}, J_{2}=5.5 \mathrm{~Hz}\right), 7.70(\mathrm{t}, 2 \mathrm{H}, J=7.7 \mathrm{~Hz}), 7.58(\mathrm{t}, 2 \mathrm{H}, J=7.6 \mathrm{~Hz}), 7.41\left(\mathrm{dd}, 2 \mathrm{H}, J_{1}=5.9 \mathrm{~Hz}, J_{2}=3.5 \mathrm{~Hz}\right), 6.94(\mathrm{~d}$, $4 \mathrm{H}, J=8.8 \mathrm{~Hz}), 6.82(\mathrm{~d}, 4 \mathrm{H}, J=8.8 \mathrm{~Hz}), 5.67\left(\mathrm{dt}, 8 \mathrm{H}, J_{1}=5.7 \mathrm{~Hz}, J_{2}=3.1 \mathrm{~Hz}\right), 5.59\left(\mathrm{dt}, 8 \mathrm{H}, J_{1}=5.7 \mathrm{~Hz}, J_{2}=3.1 \mathrm{~Hz}\right)$, 4.97 (s, 4H), 1.70 (s, 6H), 1.59-1.36 (m, 24H), 1.29 (s, 12H). $\left.{ }^{13} \mathrm{C} \mathrm{NMR} \mathrm{(100} \mathrm{MHz,} \mathrm{CDCl}_{3}\right): \delta 172.7,147.6,144.5,139.3$, 138.7, 138.3, 137.9, 131.7, 130.1, 129.4, 129.3, 127.8, 127.5, 127.2, 126.8, 125.7, 124.6, 123.3, 116.5, 105.1, 103.2, 103.2, 102.9, 67.2, 47.5, 35.4, 29.8, 29.5, 29.0, 28.5, 25.4, 25.2. HRMS (ESI+) $m / z:[\mathrm{M}+\mathrm{K}]^{+}$calcd for $\mathrm{C}_{90} \mathrm{H}_{90} \mathrm{~N}_{10} \mathrm{O}_{4} \mathrm{~K}$, 1413.6778; found, 1413.6766 .

DPAC-monoC4P: ${ }^{1} \mathrm{H}$ NMR (400 MHz, DMSO- $\left.d_{6}\right): \delta 9.52$ (s, $\left.2 \mathrm{H}\right), 9.30$ (s, $\left.2 \mathrm{H}\right), 8.94$ (d, 2H, $J=8.6 \mathrm{~Hz}$ ), 7.96-7.88 (m, 4H), $7.71(\mathrm{t}, 2 \mathrm{H}, J=8.2 \mathrm{~Hz}), 7.61(\mathrm{t}, 2 \mathrm{H}, J=7.1 \mathrm{~Hz}), 7.44-7.40(\mathrm{~m}, 2 \mathrm{H}), 7.11-7.07(\mathrm{~m}, 2 \mathrm{H}), 6.99\left(\mathrm{dd}, 4 \mathrm{H}, J_{1}=17.5 \mathrm{~Hz}\right.$, $\left.J_{2}=8.3 \mathrm{~Hz}\right), 6.85-6.83(\mathrm{~m}, 3 \mathrm{H}), 5.69-5.66(\mathrm{~m}, 4 \mathrm{H}), 5.59\left(\mathrm{dd}, 4 \mathrm{H}, J_{1}=13.0 \mathrm{~Hz}, J_{2}=3.0 \mathrm{~Hz}\right), 4.98(\mathrm{~s}, 2 \mathrm{H}), 1.71(\mathrm{~s}, 3 \mathrm{H})$, 1.49 (d, $12 \mathrm{H}, J=30.9 \mathrm{~Hz}), 1.29$ (s, 6H). ${ }^{13} \mathrm{C} \mathrm{NMR}\left(100 \mathrm{MHz}, \mathrm{CDCl}_{3}\right): \delta 172.7,147.6,145.0,139.3,138.7,138.4,138.3$, $131.8,130.0,129.5,129.4,129.0,127.6,127.5,127.4,127.2,126.7,126.7,125.7,125.5,124.8,124.6,123.2,123.2$, 121.4, 117.1, 116.4, 105.1, 103.2, 103.1, 102.9, 67.2, 47.5, 35.3, 29.7, 29.4, 29.0, 28.5, 25.4, 25.2. HRMS (ESI+) $m / z$ : $[\mathrm{M}+\mathrm{K}]^{+}$calcd for $\mathrm{C}_{61} \mathrm{H}_{56} \mathrm{~N}_{6} \mathrm{O}_{2} \mathrm{~K}, 943.4096$; found, 943.4088 . 


\section{UV-vis and fluorescent spectral response to linear saturated dicarboxylates $\left(\mathrm{Cn}^{2-}\right)$}
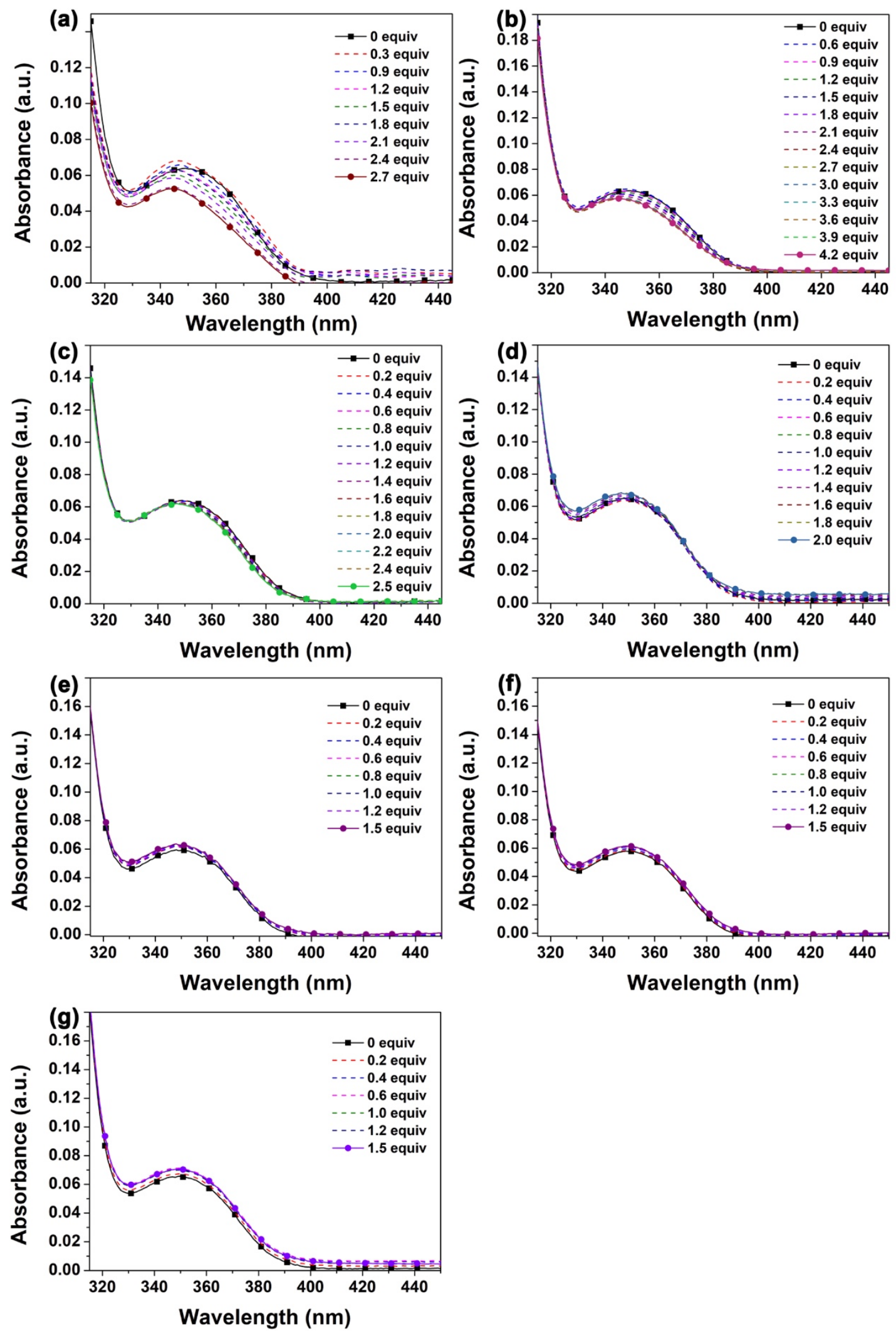

Figure S1. UV-vis spectral titrations of DPAC-bisC4P showing the spectral response produced upon the addition of (a) $\mathbf{C 2}^{2-}$, (b) $\mathbf{C 4}^{2-}$, (c) $\mathbf{C 5}^{2-}$, (d) $\mathbf{C 6}^{2-}$, (e) $\mathbf{C 7}^{2-}$, (f) $\mathbf{C 8}^{2-}$, and (g) $\mathbf{C 1 0}^{2-}$. [DPAC-bisC4P]: $10 \mu \mathrm{M}$; solvent: acetonitrile. 

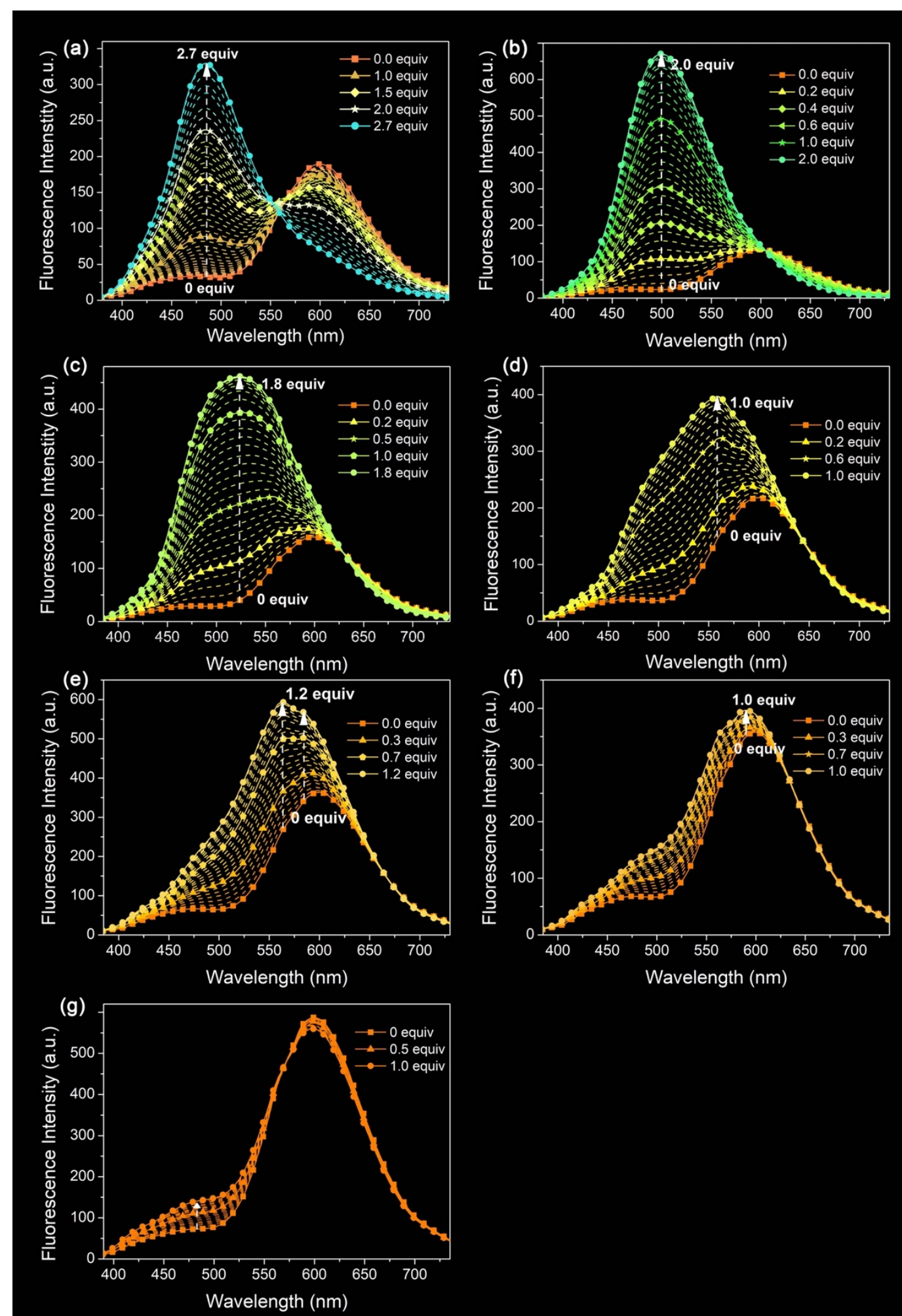

Figure S2. Fluorescence titrations of DPAC-bisC4P with (a) $\mathbf{C 2}^{2-}$, (b) $\mathbf{C 5}^{2-}$, (c) $\mathbf{C 6}^{2-}$, (d) $\mathbf{C 7}^{2-}$, (e) $\mathbf{C 8}^{2-}$, (f) $\mathbf{C 1 0}^{2-}$, and (g) C122-. $\lambda_{\text {ex }}: 350 \mathrm{~nm}$; [DPAC-bisC4P]: $10 \mu \mathrm{M}$; solvent: acetonitrile. 


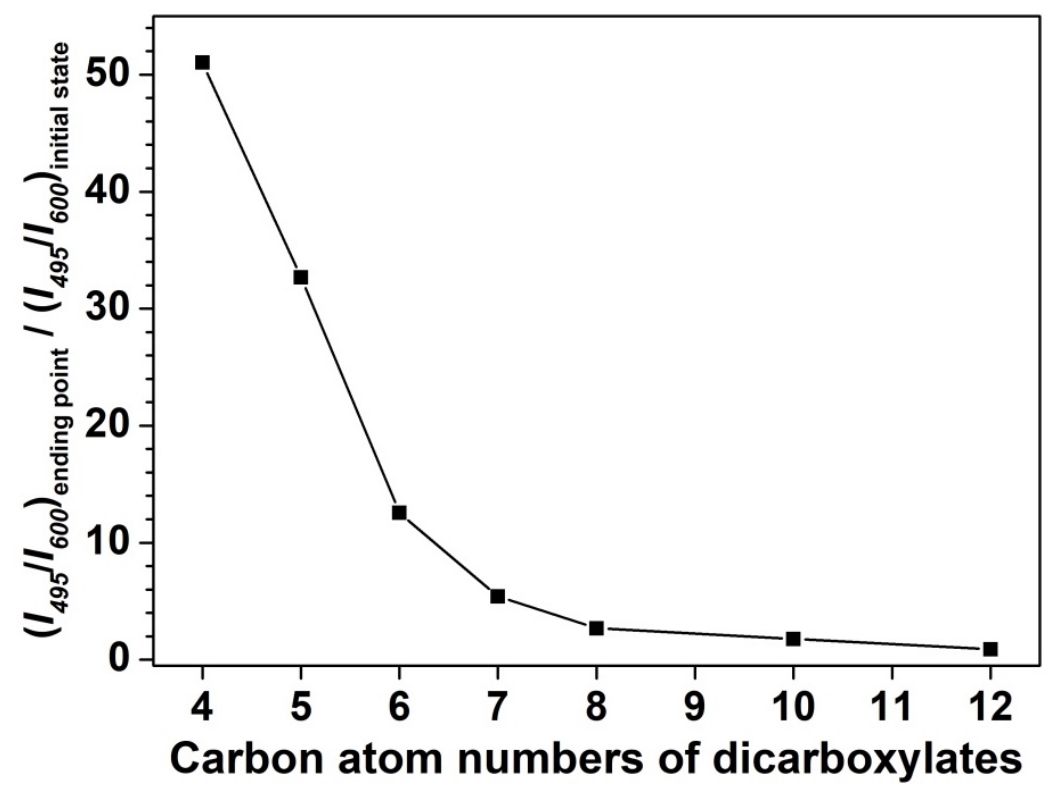

Figure S3. Plot of the intensity ratios $I_{495} / I_{600}$ recorded at the end point of the titrations shown in Figure 3 and Figure S2 vs the chain lengths of linear dicarboxylates (given as the number of carbon atoms in the dianion under consideration).
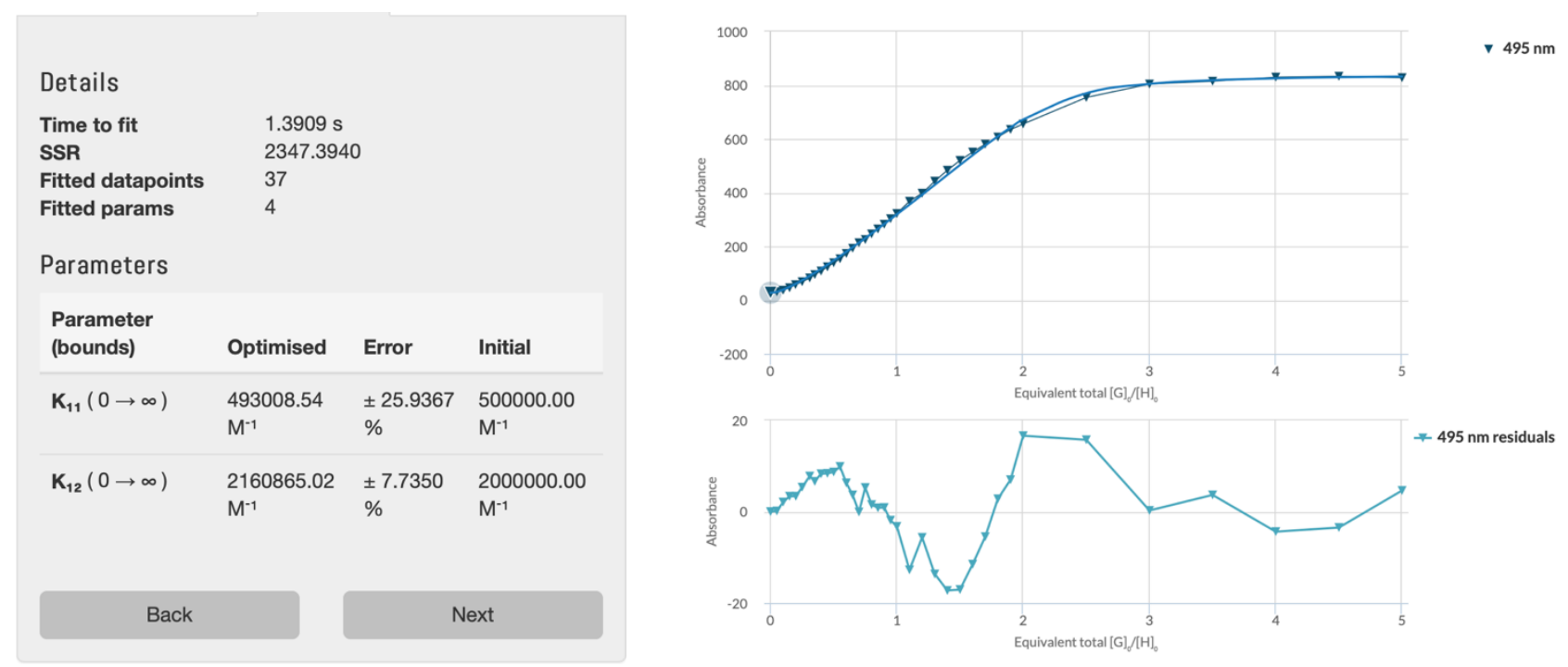

Figure S4. Least-squares nonlinear fitting of the normalized change in fluorescence at $495 \mathrm{~nm}$ obtained as a function of concentration on the basis of the fluorescence titration of DPAC-bisC4P with $\mathbf{C 4}^{2-}$. The solid lines were obtained from nonlinear curve-fitting to a 1:2 binding model using the www.supramolecular.org web applet. 


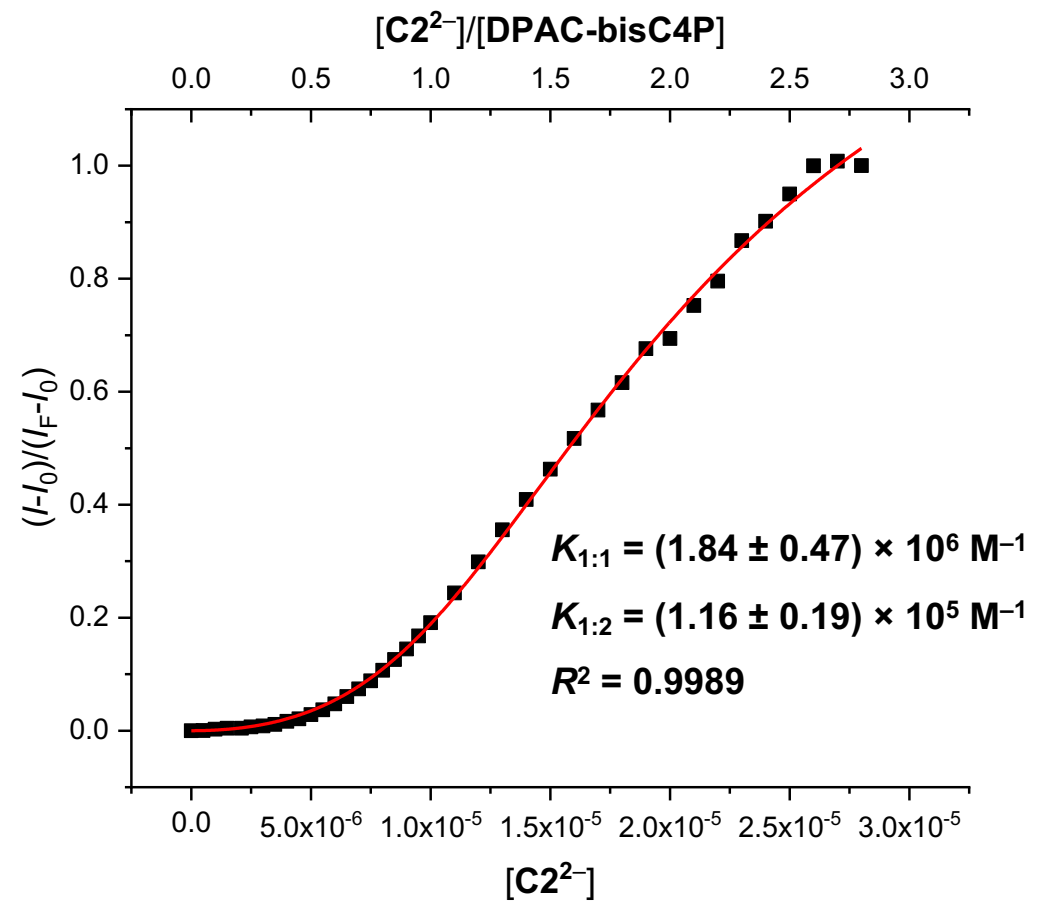

Figure S5. Least-squares nonlinear fitting of the normalized change in fluorescence at $486 \mathrm{~nm}$ as a function of concentration on the basis of the fluorescence titration of DPAC-bisC4P with $\mathbf{C 2}^{2-}$. The lower $x$ axis gives the absolute concentration of the dianion, whereas the upper $x$ axis shows the molar ratio of the dianion guest relative to that of the DPAC-bisC4P host. [DPAC-bisC4P]: $10 \mu \mathrm{M}$; solvent: acetonitrile.
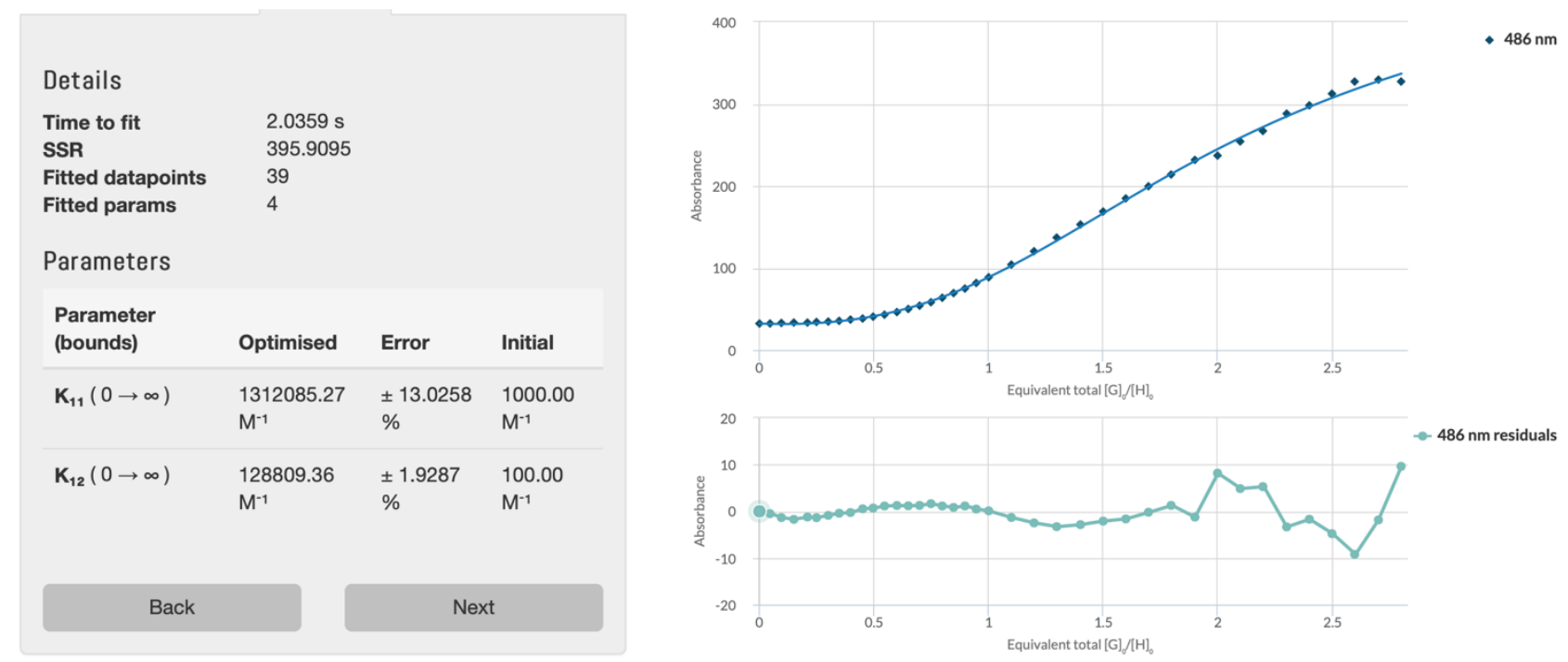

Figure S6. Least-squares nonlinear fitting of the normalized change in fluorescence at $486 \mathrm{~nm}$ obtained as a function of concentration on the basis of the fluorescence titration of DPAC-bisC4P with $\mathbf{C 2}^{2-}$. The solid lines were obtained from nonlinear curve-fitting to a 1:2 binding model using the www.supramolecular.org web applet. 


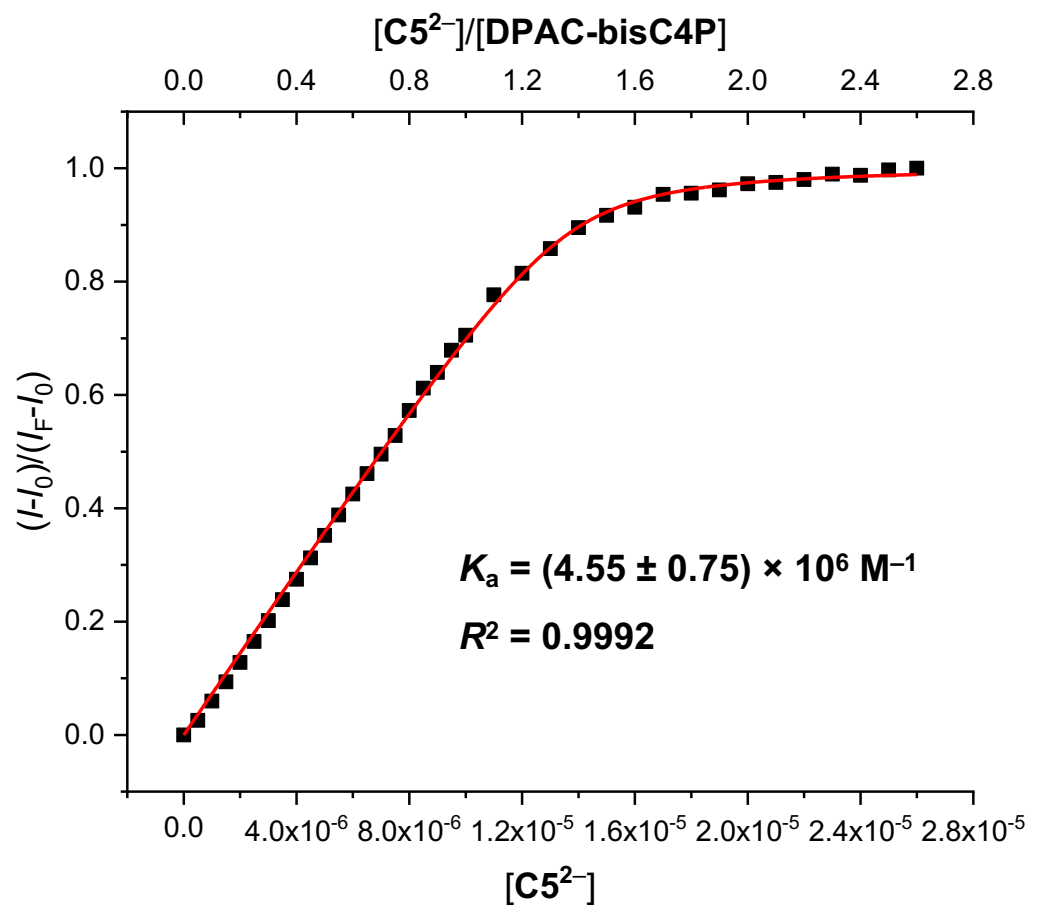

Figure S7. Least-squares nonlinear fitting of the normalized change in fluorescence at $500 \mathrm{~nm}$ as a function of concentration on the basis of the fluorescence titration of DPAC-bisC4P with $\mathbf{C 5}^{2-}$. The lower $x$ axis gives the absolute concentration of the dianion, whereas the upper $x$ axis shows the molar ratio of the dianion guest relative to that of the DPAC-bisC4P host. [DPAC-bisC4P]: $10 \mu \mathrm{M}$; solvent: acetonitrile.

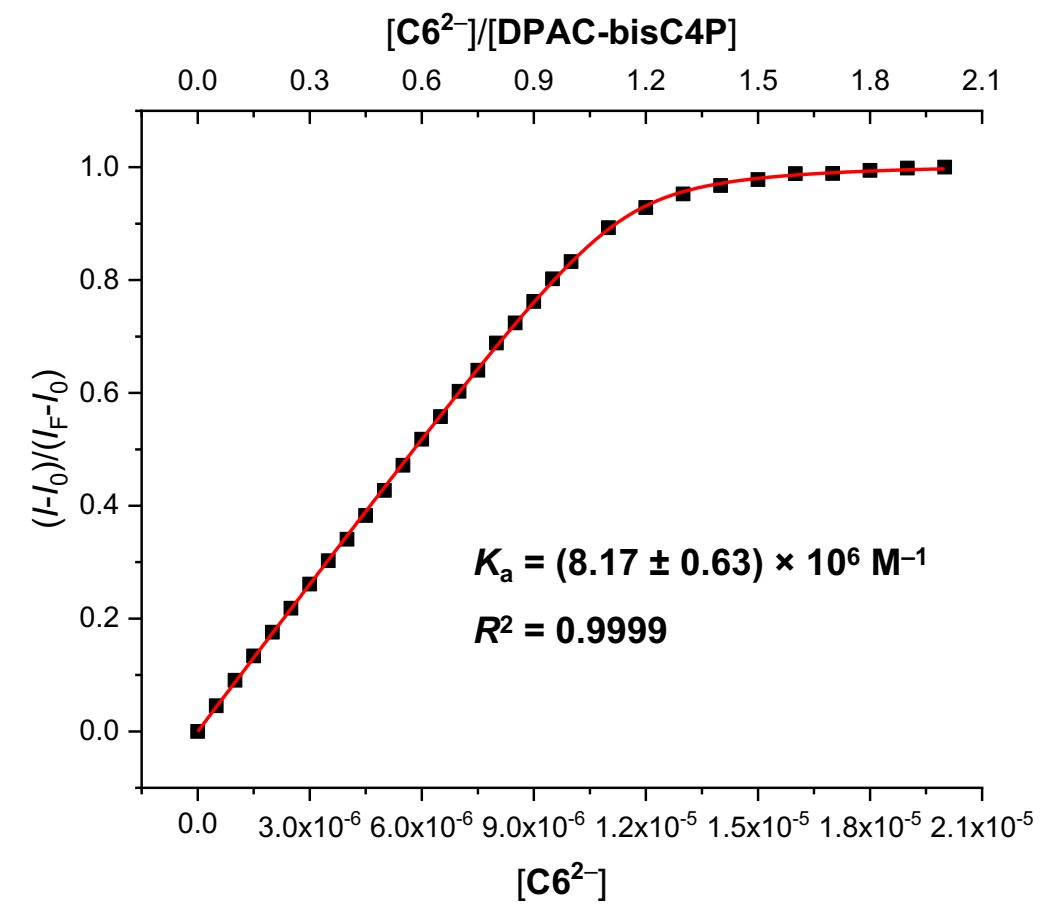

Figure S8. Least-squares nonlinear fitting of the normalized change in fluorescence at $525 \mathrm{~nm}$ as a function of concentration on the basis of the fluorescence titration of DPAC-bisC4P with $\mathbf{C 6}^{2-}$. The lower $x$ axis gives the absolute concentration of the dianion, whereas the upper $x$ axis shows the molar ratio of the dianion guest relative to that of the DPAC-bisC4P host. [DPAC-bisC4P]: $10 \mu \mathrm{M}$; solvent: acetonitrile. 


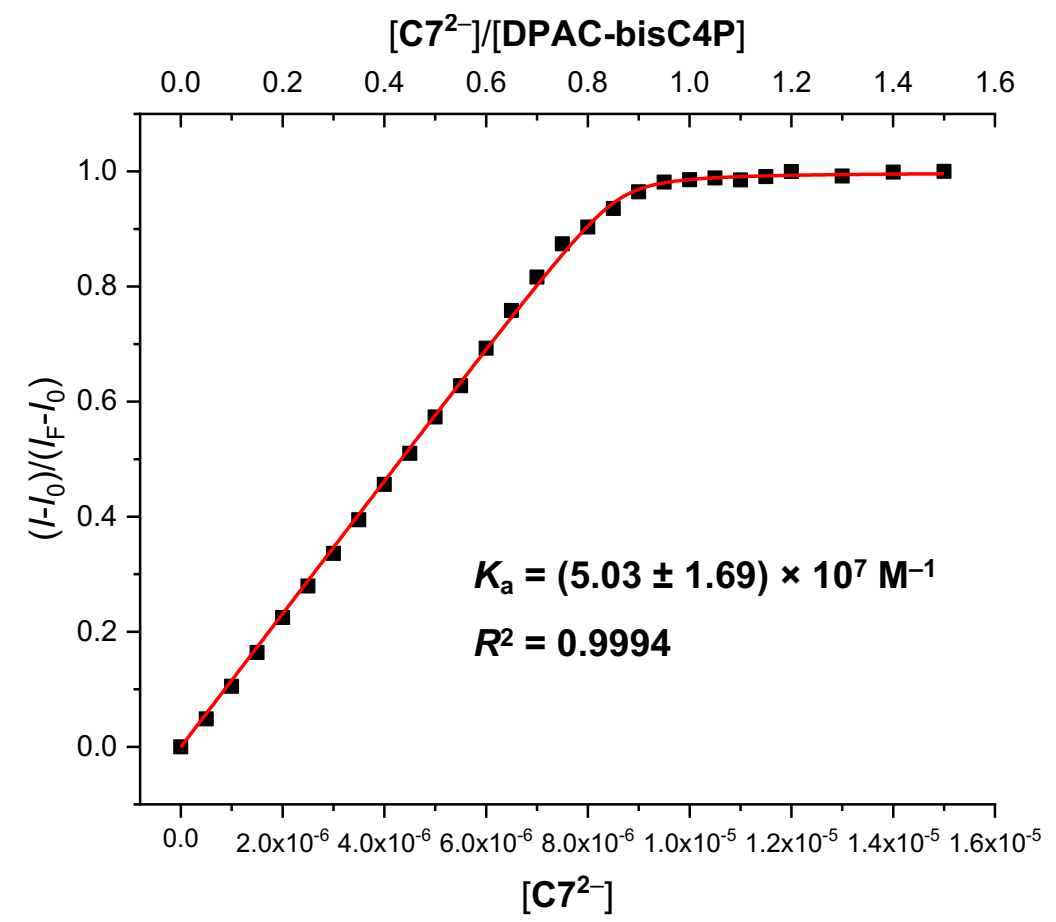

Figure S9. Least-squares nonlinear fitting of the normalized change in fluorescence at $560 \mathrm{~nm}$ as a function of concentration on the basis of the fluorescence titration of DPAC-bisC4P with $\mathbf{C 7}^{2-}$. The lower $x$ axis gives the absolute concentration of the dianion, whereas the upper $x$ axis shows the molar ratio of the dianion guest relative to that of the DPAC-bisC4P host. [DPAC-bisC4P]: $10 \mu \mathrm{M}$; solvent: acetonitrile.

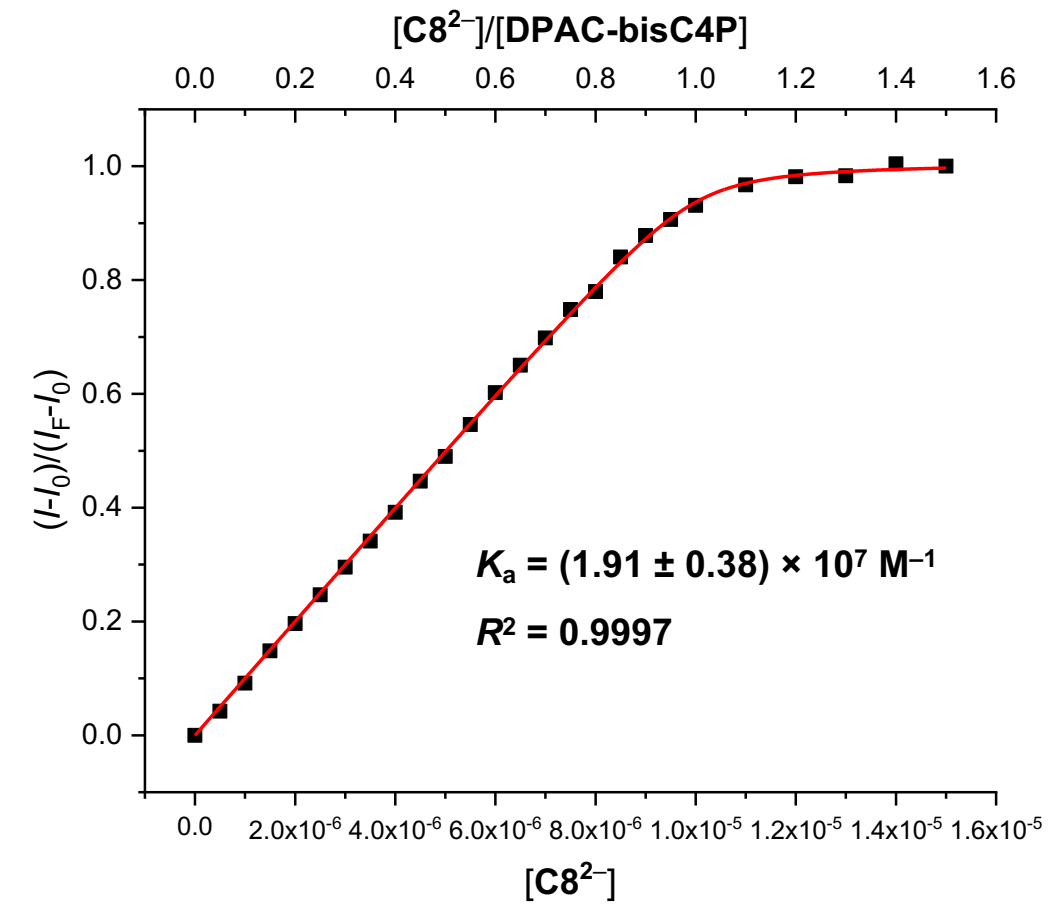

Figure S10. Least-squares nonlinear fitting of the normalized change in fluorescence at $563 \mathrm{~nm}$ as a function of concentration on the basis of the fluorescence titration of DPAC-bisC4P with $\mathbf{C 8}^{2-}$. The lower $x$ axis gives the absolute concentration of the dianion, whereas the upper $x$ axis shows the molar ratio of the dianion guest relative to that of the DPAC-bisC4P host. [DPAC-bisC4P]: $10 \mu \mathrm{M}$; solvent: acetonitrile. 


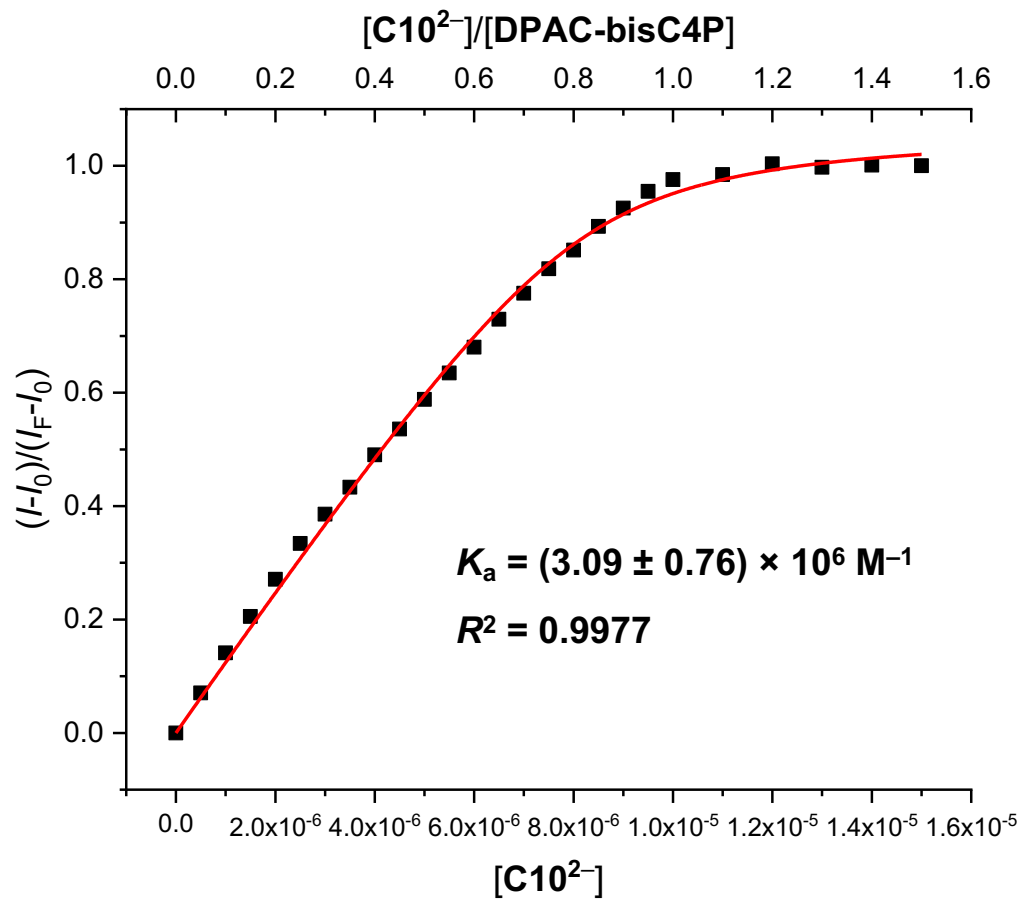

Figure S11. Least-squares nonlinear fitting of the normalized change in fluorescence at $485 \mathrm{~nm}$ as a function of concentration on the basis of the fluorescence titration of DPAC-bisC4P with $\mathbf{C 1 0}^{\mathbf{2}}$. The lower $x$ axis gives the absolute concentration of the dianion, whereas the upper $x$ axis shows the molar ratio of the dianion guest relative to that of the DPAC-bisC4P host. [DPAC-bisC4P]: $10 \mu \mathrm{M}$; solvent: acetonitrile.

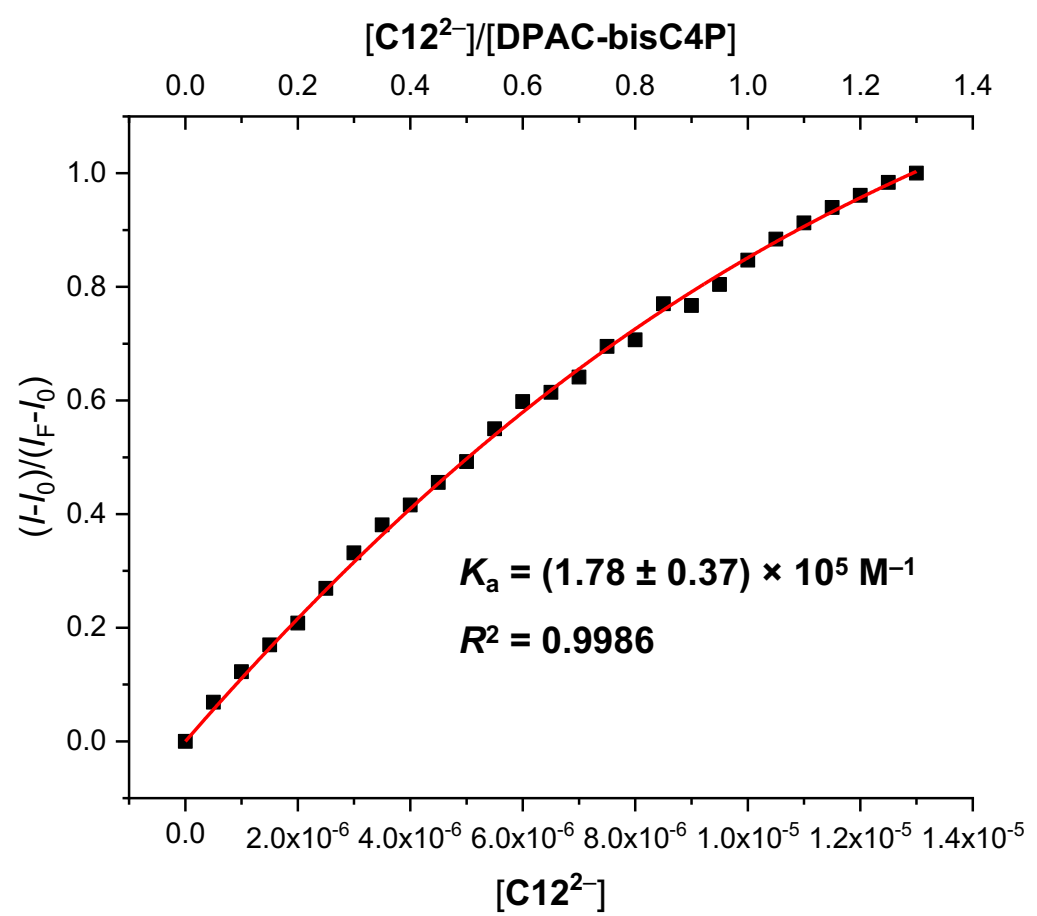

Figure S12. Least-squares nonlinear fitting of the normalized change in fluorescence at $485 \mathrm{~nm}$ as a function of concentration on the basis of the fluorescence titration of DPAC-bisC4P with $\mathbf{C 1 2}^{\mathbf{2}}$. The lower $x$ axis gives the absolute concentration of the dianion, whereas the upper $x$ axis shows the molar ratio of the dianion guest relative to that of the DPAC-bisC4P host. [DPAC-bisC4P]: $10 \mu \mathrm{M}$; solvent: acetonitrile. 


\section{Determination of the binding stoichiometry}

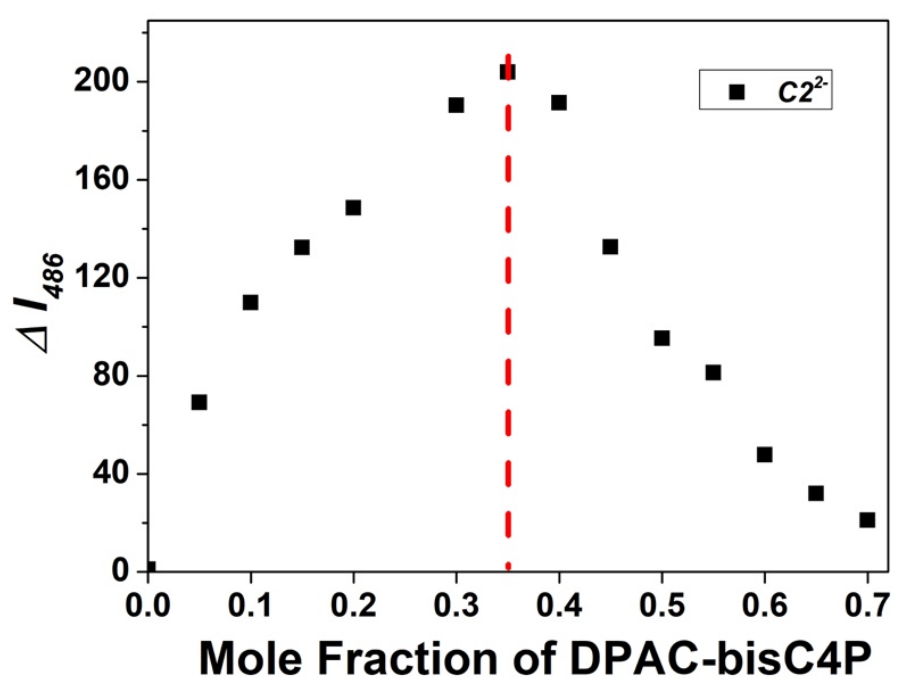

Figure S13. Job plot analysis of the presumed DPAC-bisC4P $\supset \mathbf{C 2}^{2-}$ complex. The $\Delta I$ values were determined at $486 \mathrm{~nm}$. The total concentration of DPAC-bisC4P and $\mathbf{C 2}^{2-}$ was held at $1.0 \times 10^{-5} \mathrm{M}$; solvent: acetonitrile.

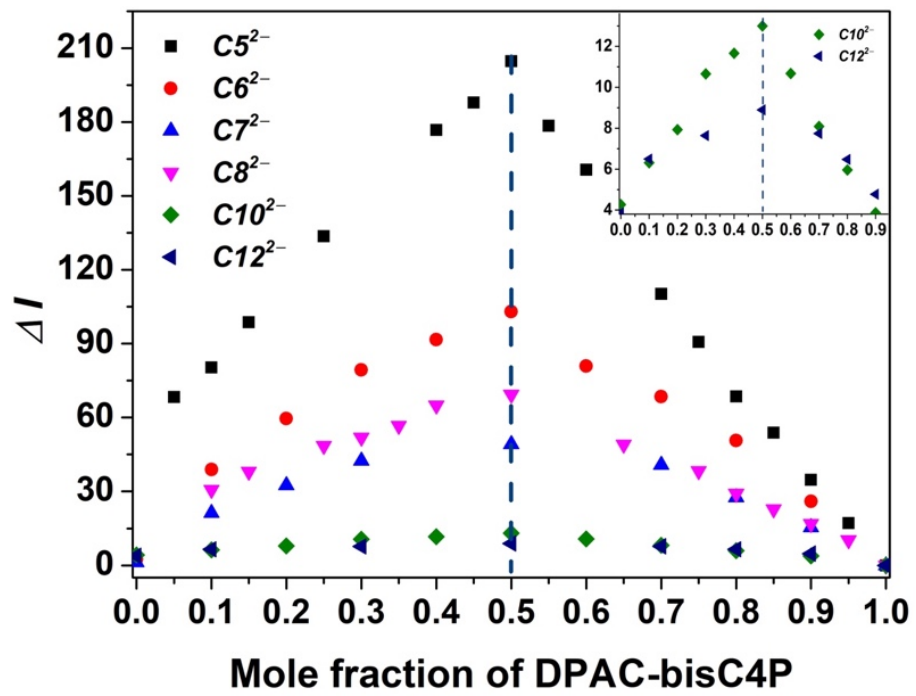

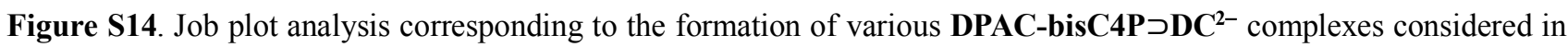
this study. The $\Delta I$ values were determined at $500 \mathrm{~nm}, 525 \mathrm{~nm}, 560 \mathrm{~nm}, 563 \mathrm{~nm}, 485 \mathrm{~nm}$, and $485 \mathrm{~nm}$ for $\mathbf{C 5}^{2-}$, $\mathbf{C 6}^{2-}$, $\mathbf{C 7}^{2-}, \mathbf{C 8}^{2-}, \mathbf{C 1 0}^{2-}$, and $\mathbf{C 1 2}^{2-}$, respectively. The total concentration of host (i.e., DPAC-bisC4P) and guest (i.e., $\mathbf{C n}{ }^{2-}$ ) was held at $1.0 \times 10^{-5} \mathrm{M}$; solvent: acetonitrile. 


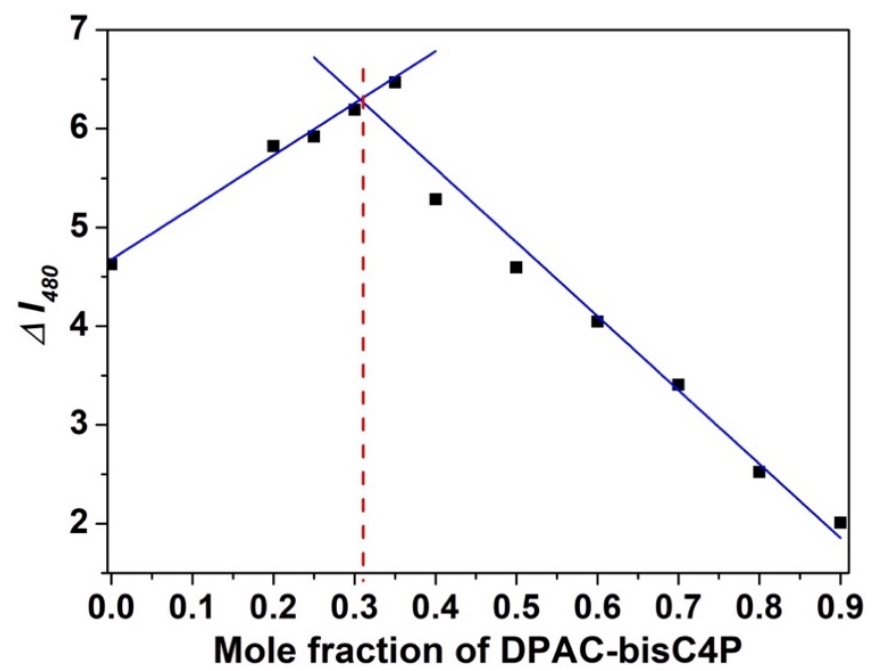

Figure S15. Job's plot corresponding to the interaction of DPAC-bisC4P with $\mathrm{TBACH}_{3} \mathrm{CO}_{2}$. The total concentration of receptor and salt $=1.0 \times 10^{-5} \mathrm{M}$ in acetonitrile.
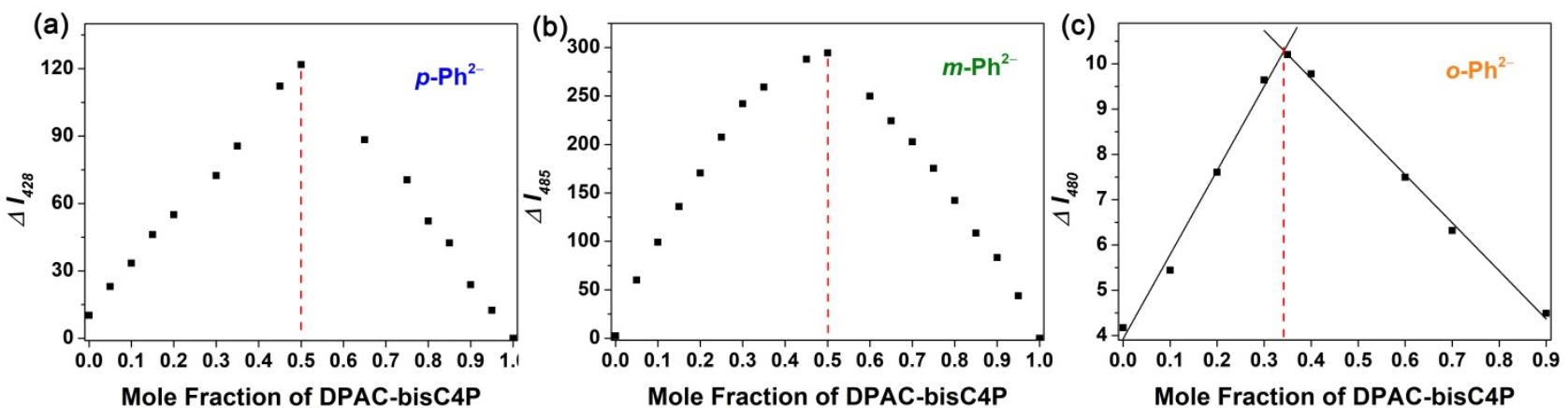

Figure S16. Job's plots corresponding to the interaction of DPAC-bisC4P with (a) $\mathbf{p}-\mathbf{P h}^{2-}$, (b) $\boldsymbol{m}-\mathbf{P h}^{2-}$, and (c) $\boldsymbol{o}-\mathbf{P h}^{2-}$. The total concentration of receptor and salt $=1.0 \times 10^{-5} \mathrm{M}$ in acetonitrile. 


\section{Fluorescent response to the three phthalate isomers}
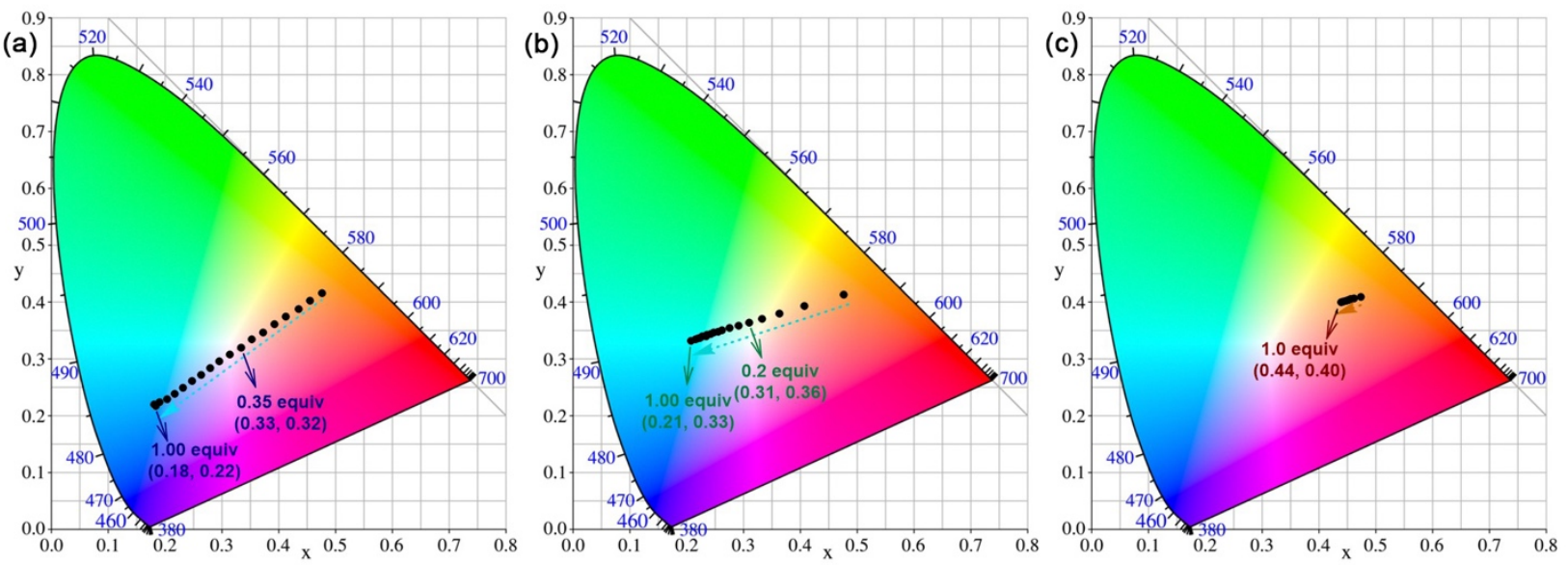

Figure S17. Chromaticity coordinates (CIE) for DPAC-bisC4P recorded in acetonitrile in the presence of varying quantities of (a) $\boldsymbol{p}-\mathbf{P h}^{2-}$, (b) $\boldsymbol{m}-\mathbf{P h}^{2-}$, and (c) $\boldsymbol{o}-\mathbf{P h}^{2-}$ (from 0 to 1.0 equiv).

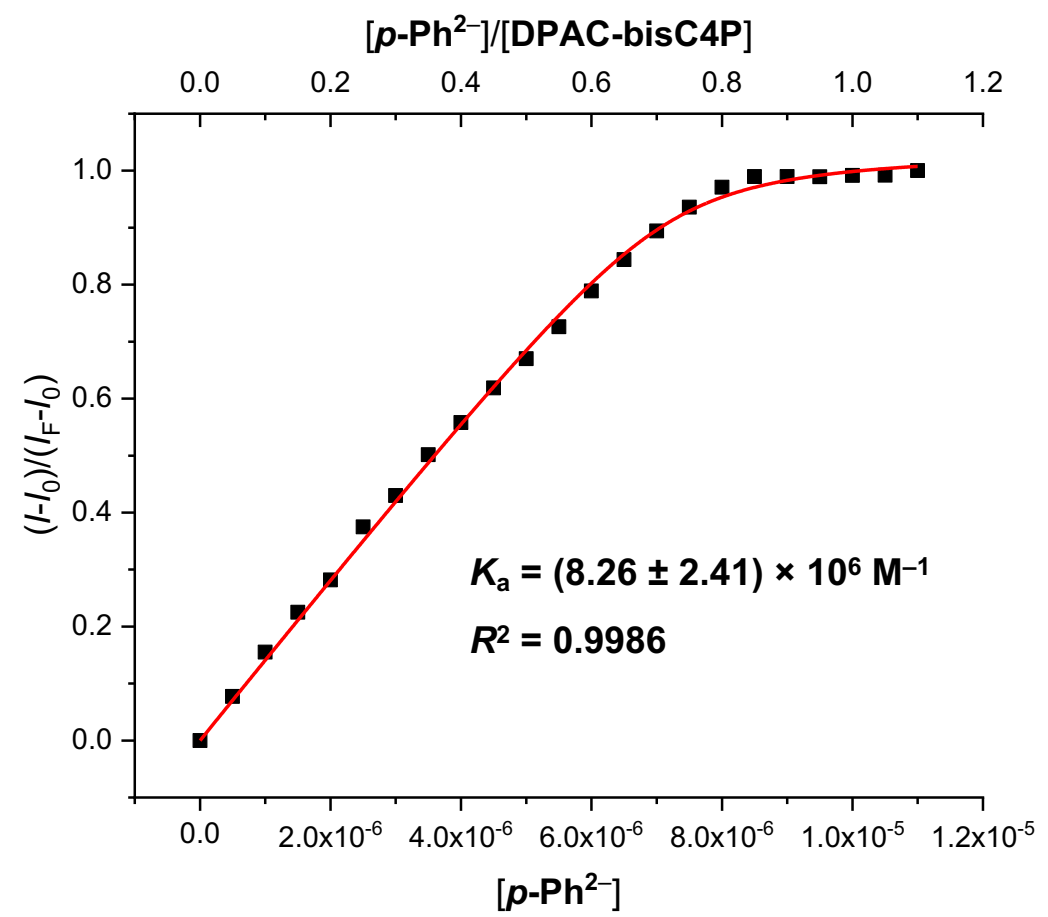

Figure S18. Least-squares nonlinear fitting of the normalized change in fluorescence at $600 \mathrm{~nm}$ as a function of concentration obtained from a fluorescence titration of DPAC-bisC4P with $\mathbf{p}-\mathbf{P h}^{2-}$. The lower $x$ axis gives the absolute concentration of the dianion, whereas the upper $x$ axis shows the molar ratio of the dianion guest relative to that of the DPAC-bisC4P host. [DPAC-bisC4P]: $10 \mu \mathrm{M}$; solvent: acetonitrile. 


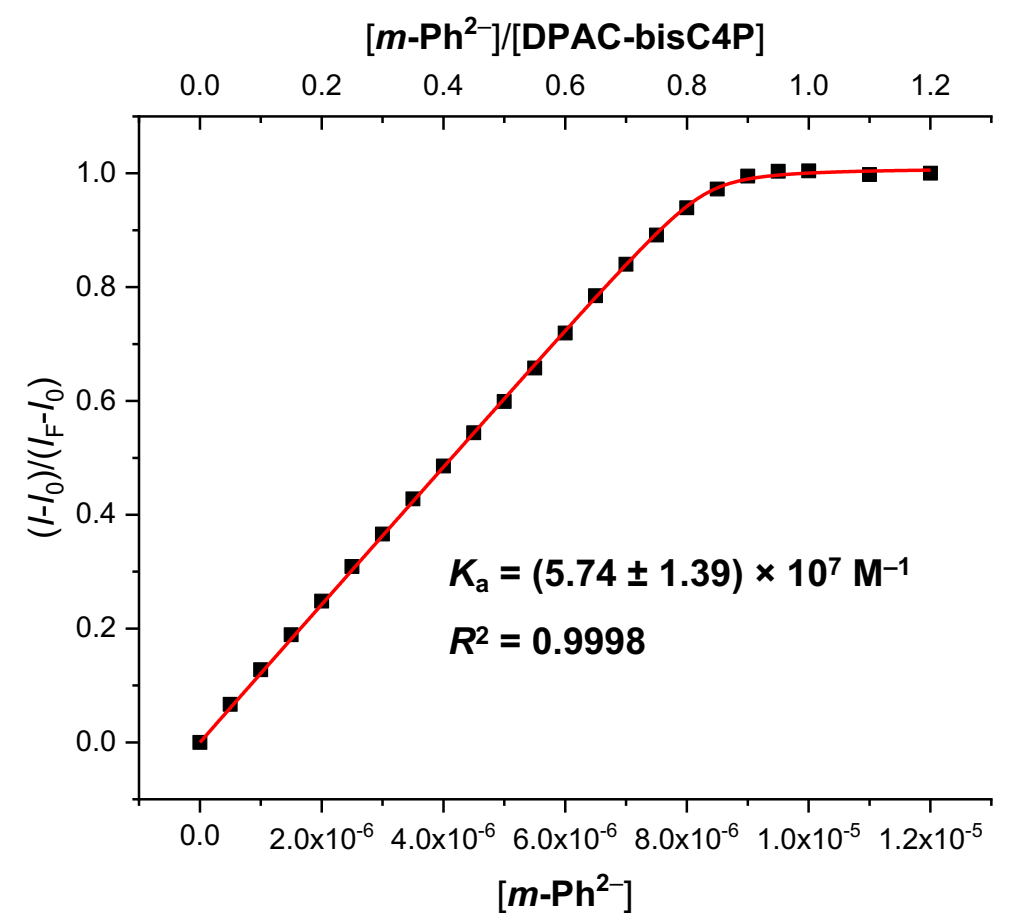

Figure S19. Least-squares nonlinear fitting of the normalized change in fluorescence at $485 \mathrm{~nm}$ as a function of concentration obtained from a fluorescence titration of DPAC-bisC4P with $\boldsymbol{m}-\mathbf{P h}^{2-}$. The lower $x$ axis gives the absolute concentration of the dianion, whereas the upper $x$ axis shows the molar ratio of the dianion guest relative to that of the DPAC-bisC4P host. [DPAC-bisC4P]: $10 \mu \mathrm{M}$; solvent: acetonitrile. 


\section{Determination of the limit of detection (LOD)}

The following formula was used to determine the limit of detection (LOD):

$$
\text { Limit of Dection }(L O D)=\frac{3 \sigma}{k}
$$

where $\sigma$ is the standard deviation of emission intensity (ratio) for the initial host solution $\left(c_{0}=1.0 \times 10^{-5} \mathrm{M}\right.$ in $3.0 \mathrm{~mL}$ of $\left.\mathrm{CH}_{3} \mathrm{CN}\right) ; k$ is the slope of the calibration curve (Figures S20-S27).

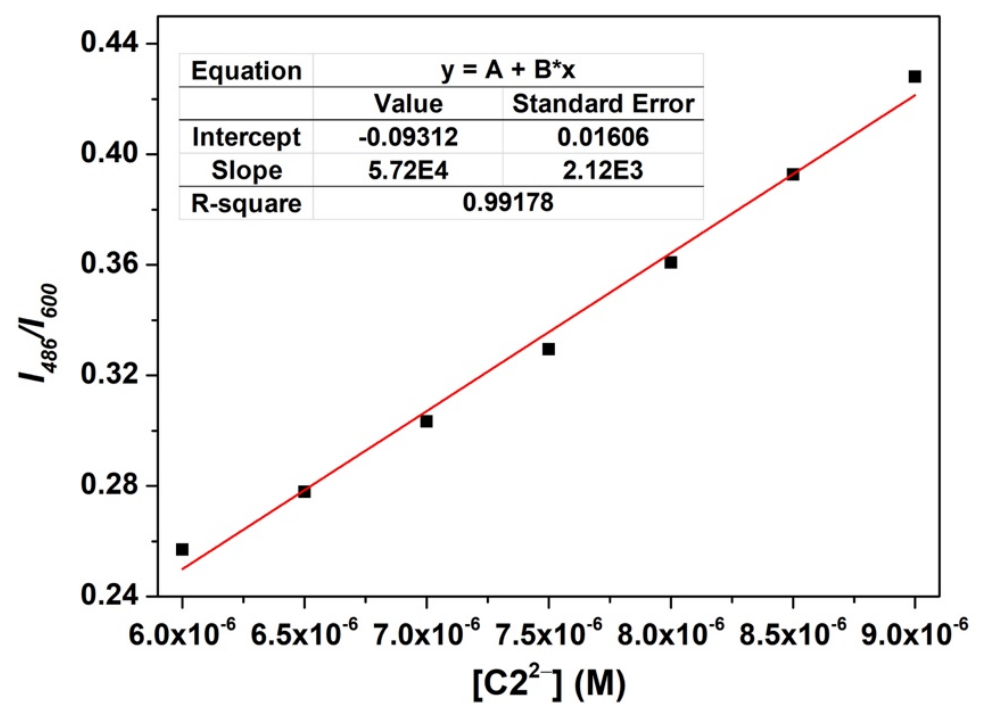

Figure S20. Plots of the emission intensity ratio $I_{486} / I_{600}$ versus the concentration of $\mathbf{C 2}^{2-}$ in acetonitrile; $\lambda_{\mathrm{ex}}: 350 \mathrm{~nm} ; \lambda_{\mathrm{em}}$ : $486 \mathrm{~nm}, 600 \mathrm{~nm} ; k=5.72 \times 10^{4} \mathrm{M}^{-1} ; \sigma=1.79 \times 10^{-3}$. The standard deviation $(\sigma)$ of the blank solution was obtained by recording the emission intensity of the pure host solution (i.e., prior adding $\mathbf{C 2}^{\mathbf{2}}$ ) 10 separate times. The limit of detection (LOD) was calculated as $93.9 \mathrm{nM}$ by the above formula.

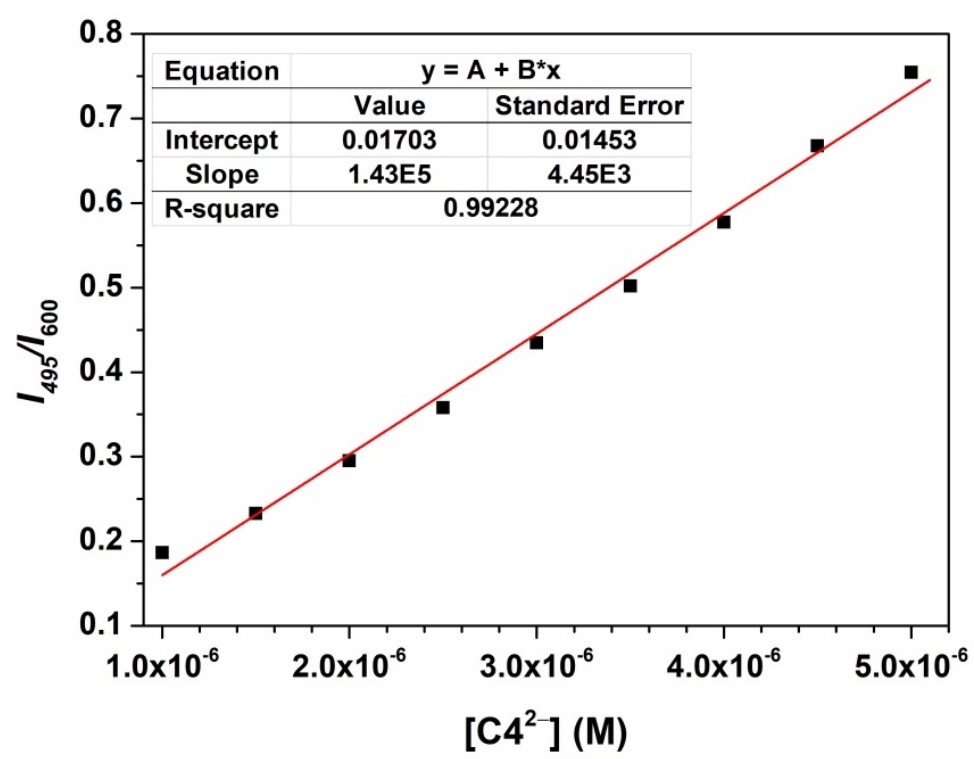

Figure S21. Plots of the emission intensity ratio $I_{495} / I_{600}$ versus the concentration of $\mathbf{C 4}^{2-}$ in acetonitrile; $\lambda_{\mathrm{ex}}: 350 \mathrm{~nm} ; \lambda_{\mathrm{em}}$ : $495 \mathrm{~nm}, 600 \mathrm{~nm} ; k=1.43 \times 10^{5} \mathrm{M}^{-1} ; \sigma=1.64 \times 10^{-3}$. The standard deviation $(\sigma)$ of the blank solution was obtained by recording the emission intensity of the pure host solution (i.e., prior adding $\mathbf{C 4}^{2-}$ ) 10 separate times. The limit of detection (LOD) was calculated as $34.4 \mathrm{nM}$ by the above formula. 


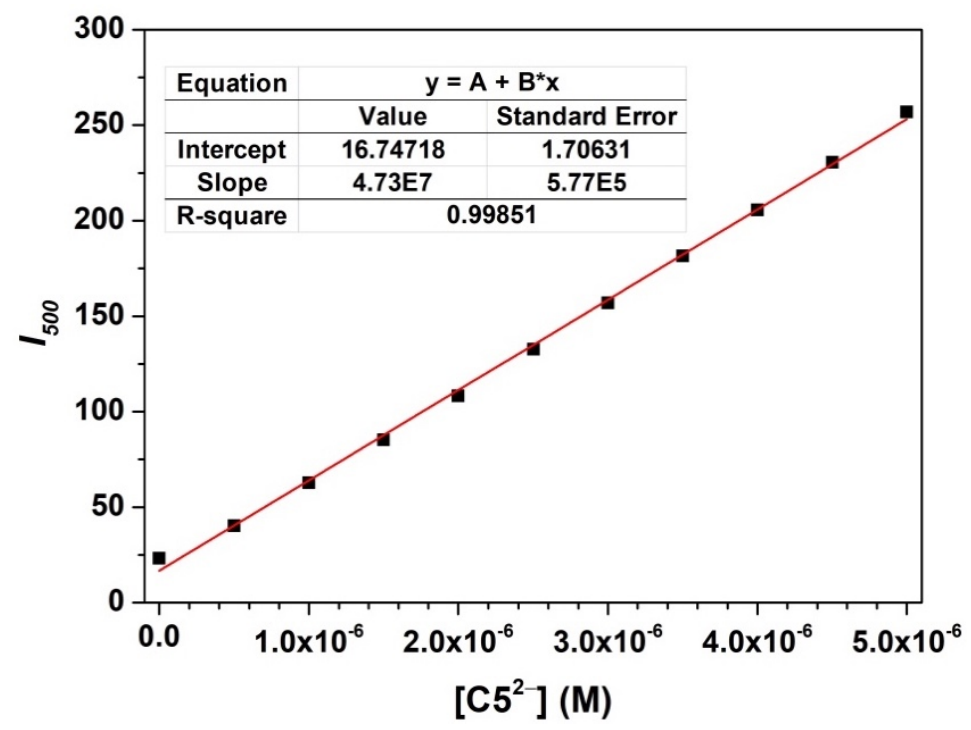

Figure S22. Plots of the emission intensity $I_{500}$ versus the concentration of $\mathbf{C 5}^{\mathbf{2}}$ in acetonitrile; $\lambda_{\mathrm{ex}}: 350 \mathrm{~nm} ; \lambda_{\mathrm{em}}: 500 \mathrm{~nm}$; $k=4.73 \times 10^{7} \mathrm{M}^{-1} ; \sigma=0.29$. The standard deviation $(\sigma)$ of the blank solution was obtained by recording the emission intensity of the pure host solution (i.e., prior adding $\mathbf{C 5}^{2-}$ ) 10 separate times. The limit of detection (LOD) was calculated as $18.4 \mathrm{nM}$ by the above formula.

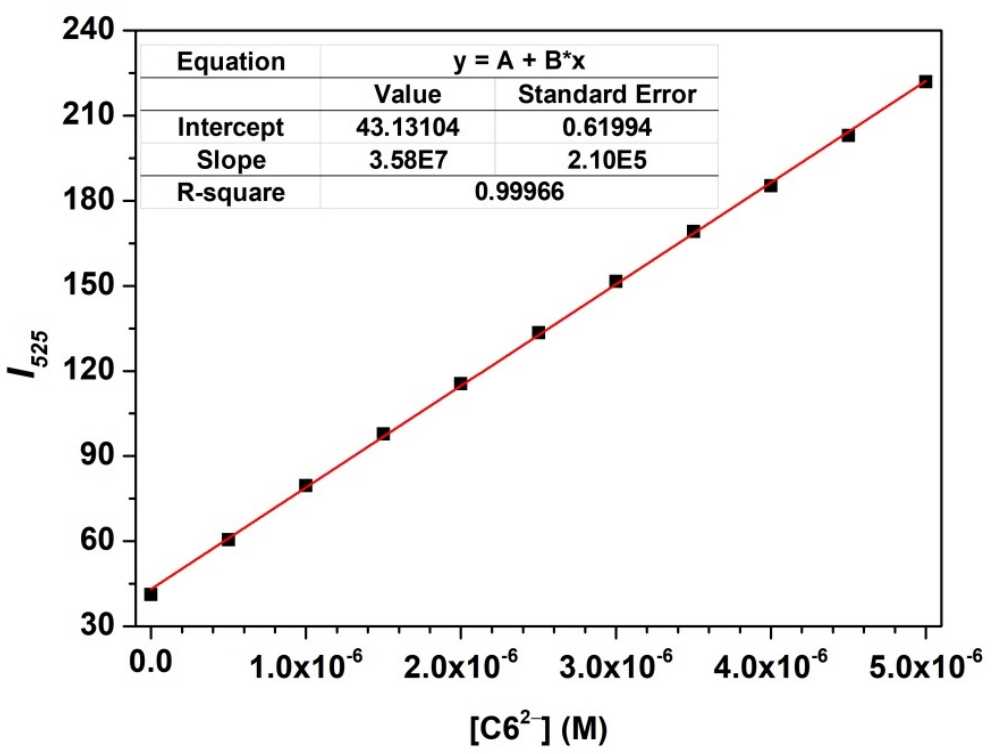

Figure S23. Plots of the emission intensity $I_{525}$ versus the concentration of $\mathbf{C 6}^{2-}$ in acetonitrile; $\lambda_{\mathrm{ex}}: 350 \mathrm{~nm} ; \lambda_{\mathrm{em}}: 525 \mathrm{~nm}$; $k=3.58 \times 10^{7} \mathrm{M}^{-1} ; \sigma=0.17$. The standard deviation $(\sigma)$ of the blank solution was obtained by recording the emission intensity of the pure host solution (i.e., prior adding $\mathbf{C 6}^{2-}$ )10 separate times. The limit of detection (LOD) was calculated as $14.2 \mathrm{nM}$ by the above formula. 


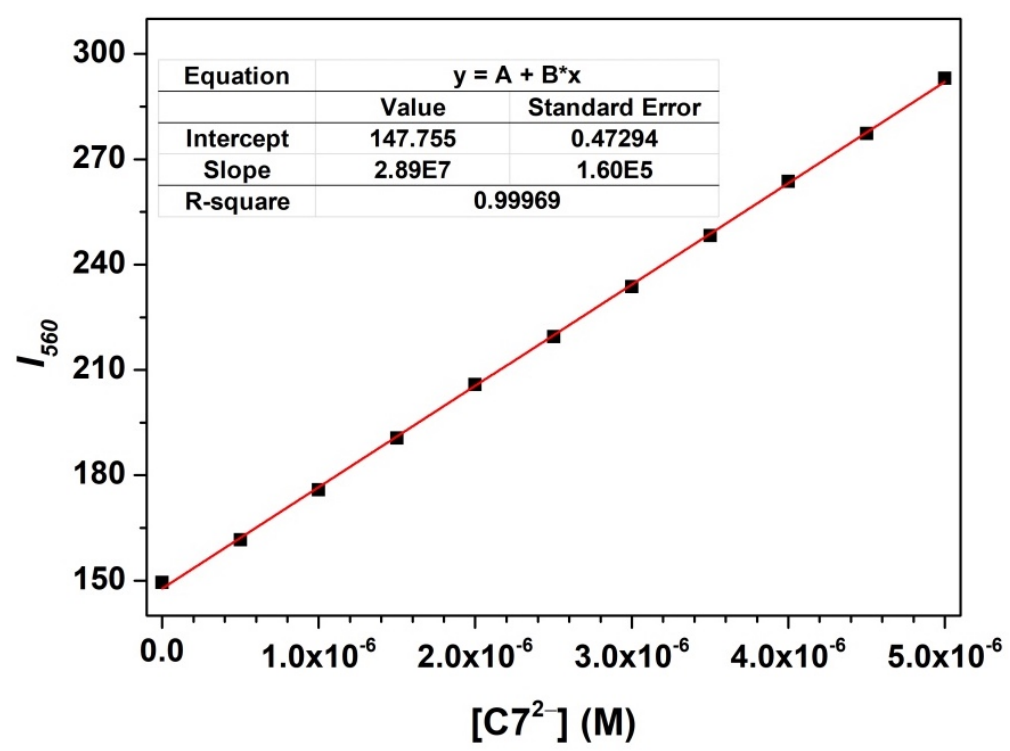

Figure S24. Plots of the emission intensity $I_{560}$ versus the concentration of $\mathbf{C 7}^{2-}$. $\lambda_{\mathrm{ex}}: 350 \mathrm{~nm} ; \lambda_{\mathrm{em}}: 560 \mathrm{~nm} ; k=2.89 \times$ $10^{7} \mathrm{M}^{-1} ; \sigma=0.58$. The standard deviation $(\sigma)$ of the blank solution was obtained by recording the emission intensity of the pure host solution (i.e., prior adding $\mathbf{C 7}^{2-}$ ) 10 separate times. The limit of detection (LOD) was calculated as $60.2 \mathrm{nM}$ by the above formula.

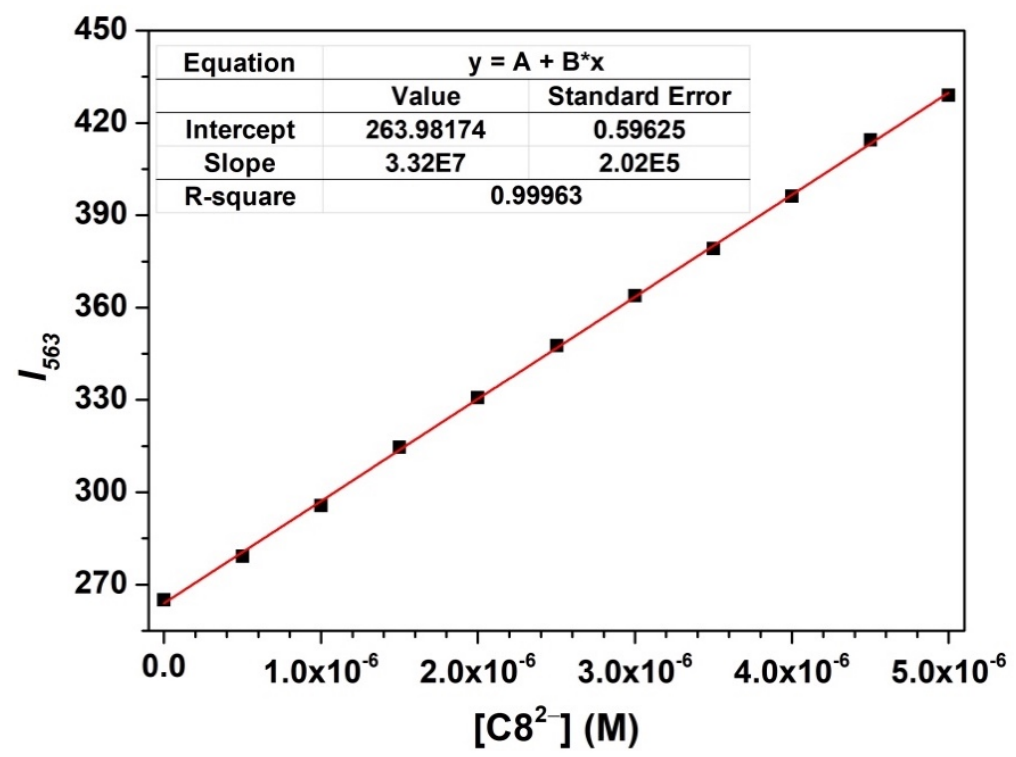

Figure S25. Plots of the emission intensity $I_{525}$ versus the concentration of $\mathbf{C 8}^{2-}$ in acetonitrile; $\lambda_{\mathrm{ex}}: 350 \mathrm{~nm} ; \lambda_{\mathrm{em}}: 563 \mathrm{~nm}$; $k=3.32 \times 10^{7} \mathrm{M}^{-1} ; \sigma=0.88$. The standard deviation $(\sigma)$ of the blank solution was obtained by recording the emission intensity of the pure host solution (i.e., prior adding $\mathbf{C 8}^{2-}$ ) 10 separate times. The limit of detection (LOD) was calculated as $79.5 \mathrm{nM}$ by the above formula. 


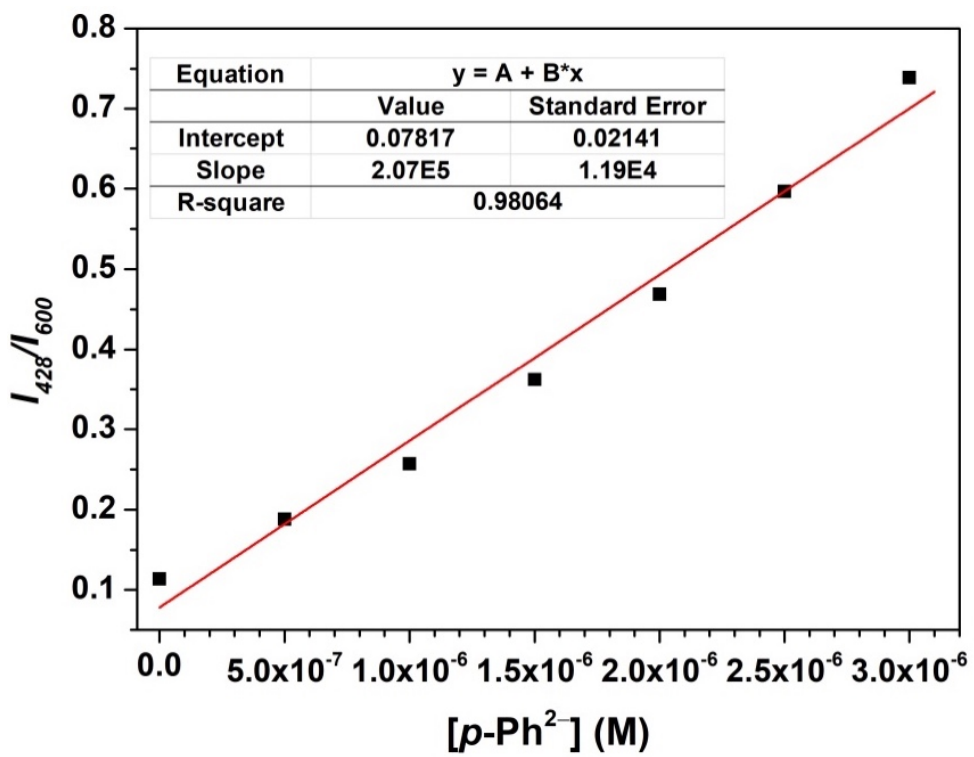

Figure S26. Plots of the emission intensity ratio $I_{428} / I_{600}$ versus the concentration of $\boldsymbol{p}$-Ph $\mathbf{P h}^{\mathbf{2}}$ in acetonitrile; $\lambda_{\mathrm{ex}}: 350 \mathrm{~nm}$; $\lambda_{\mathrm{em}}: 428 \mathrm{~nm}, 600 \mathrm{~nm} ; k=2.07 \times 10^{5} \mathrm{M}^{-1} ; \sigma=1.45 \times 10^{-3}$. The standard deviation $(\sigma)$ of the blank solution was obtained by recording the emission intensity of the pure host solution (i.e., prior adding $\mathbf{p}$-Ph $\mathbf{h}^{\mathbf{2}}$ ) 10 separate times. The limit of detection (LOD) was calculated as $21.0 \mathrm{nM}$ by the above formula.

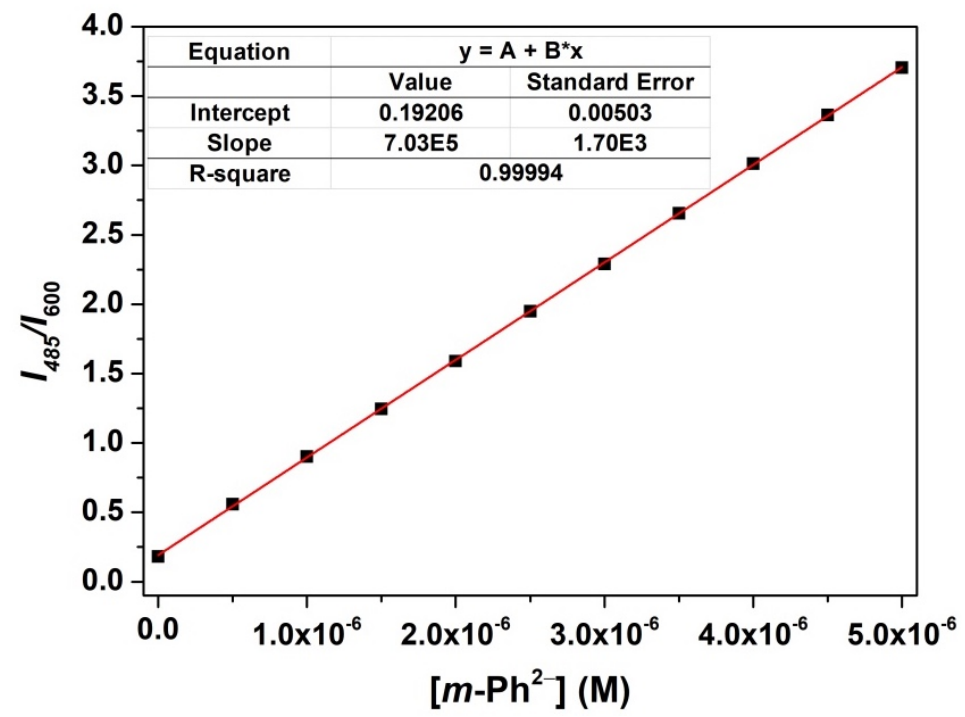

Figure S27. Plots of the emission intensity ratio $I_{485} / I_{600}$ versus the concentration of $\boldsymbol{m}-\mathbf{P h}^{\mathbf{2}}$ in acetonitrile; $\lambda_{\mathrm{ex}}: 350 \mathrm{~nm}$; $\lambda_{\mathrm{em}}: 485 \mathrm{~nm}, 600 \mathrm{~nm} ; k=7.03 \times 10^{5} \mathrm{M}^{-1} ; \sigma=1.77 \times 10^{-3}$. The standard deviation $(\sigma)$ of the blank solution was obtained by recording the emission intensity of the pure host solution (i.e., prior adding $\boldsymbol{m}-\mathbf{P h}^{\mathbf{2}}$ ) 10 separate times. The limit of detection (LOD) was calculated as $7.55 \mathrm{nM}$ by the above formula. 


\section{Computational studies}

Table S1. Distance between the two calix[4]pyrrole moieties $d_{\mathrm{C} 4 \mathrm{P}-\mathrm{C} 4 \mathrm{P}}$ and angles $\Theta_{\mathrm{b}}, \Theta_{\mathrm{S} 1}$, and $\Theta_{\mathrm{S} 2}$ in the present complexes based on the optimized structures of the host-guest complexes.

\begin{tabular}{|c|c|c|c|c|}
\hline Host-Guest Complexes & $d_{\mathrm{C} 4 \mathrm{P}-\mathrm{C} 4 \mathrm{P}}$ & $\Theta_{\mathbf{b}}$ & $\Theta_{\mathrm{S} 1}$ & $\Theta_{\mathrm{s} 2}$ \\
\hline DPAC-bisC4P $\supset C 2^{2-}$ & $6.1 \AA$ & $137^{\circ}$ & $138^{\circ}$ & $140^{\circ}$ \\
\hline DPAC-bisC4P $\supset C 4^{2-}$ & $8.1 \AA$ & $138^{\circ}$ & $141^{\circ}$ & $140^{\circ}$ \\
\hline DPAC-bisC4P $\supset C 5^{2-}$ & $8.6 \AA$ & $141^{\circ}$ & $143^{\circ}$ & $143^{\circ}$ \\
\hline DPAC-bisC4P $\supset C 6^{2-}$ & $10.5 \AA$ & $139^{\circ}$ & $142^{\circ}$ & $141^{\circ}$ \\
\hline DPAC-bisC4P $\supset C 7^{2-}$ & $11.8 \AA$ & $141^{\circ}$ & $144^{\circ}$ & $143^{\circ}$ \\
\hline DPAC-bisC4P $\supset C 8^{2-}$ & $13.7 \AA$ & $138^{\circ}$ & $139^{\circ}$ & $143^{\circ}$ \\
\hline DPAC-bisC4P $\supset C 10^{2-}$ & $15.6 \AA$ & $142^{\circ}$ & $144^{\circ}$ & $144^{\circ}$ \\
\hline DPAC-bisC4P $\supset C 12^{2-}$ & $17.9 \AA$ & $143^{\circ}$ & $144^{\circ}$ & $145^{\circ}$ \\
\hline DPAC-bisC4P $\supset m-\mathbf{P h}^{2-}$ & $10.4 \AA$ & $140^{\circ}$ & $142^{\circ}$ & $142^{\circ}$ \\
\hline DPAC-bisC4Pכp-Ph ${ }^{2-}$ & $10.5 \AA$ & $139^{\circ}$ & $143^{\circ}$ & $142^{\circ}$ \\
\hline
\end{tabular}

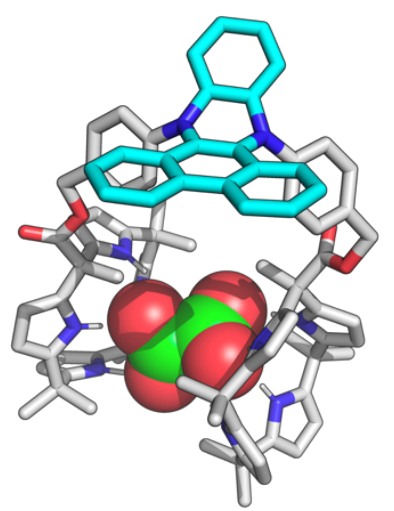

Figure S28. Optimized structure for DPAC-bisC4P $\supset \mathrm{C2}^{2-}$.

Cartesian coordinates of the optimized complex DPAC-bisC4P $\supset \mathbf{C 2}^{2-}$ :

\begin{tabular}{|c|c|c|c|c|c|c|c|}
\hline Symbol & $\mathrm{X}$ & $Y$ & Z & Symb & $\mathrm{X}$ & $Y$ & Z \\
\hline $\mathrm{C}$ & -2.714929 & -2.221812 & -0.290276 & $\mathrm{C}$ & -4.679435 & 1.928135 & -3.706133 \\
\hline $\mathrm{C}$ & -3.583824 & -2.419836 & 0.786495 & $\mathrm{~N}$ & -2.832838 & 0.054919 & -5.252535 \\
\hline $\mathrm{H}$ & -3.719486 & -3.333127 & 1.335333 & $\mathrm{C}$ & -3.712580 & 1.002832 & -5.788697 \\
\hline $\mathrm{C}$ & 3.873311 & 4.722654 & -7.000854 & $\mathrm{C}$ & -4.083446 & 0.566127 & -7.065281 \\
\hline $\mathrm{H}$ & 3.269685 & 5.569443 & -7.341138 & $\mathrm{H}$ & -4.746572 & 1.058401 & -7.739659 \\
\hline $\mathrm{H}$ & 3.965971 & 4.012335 & -7.826975 & $\mathrm{C}$ & -3.413918 & -0.664623 & -7.315413 \\
\hline $\mathrm{H}$ & 4.873953 & 5.096680 & -6.760235 & $\mathrm{H}$ & -3.494354 & -1.242619 & -8.207847 \\
\hline $\mathrm{C}$ & -4.276309 & -1.200277 & 1.020042 & $\mathrm{C}$ & -2.641187 & -0.968315 & -6.189177 \\
\hline $\mathrm{H}$ & -5.009818 & -1.037925 & 1.776696 & $\mathrm{C}$ & -4.082089 & 2.271547 & -5.051837 \\
\hline $\mathrm{C}$ & -3.824073 & -0.266791 & 0.077889 & $\mathrm{C}$ & -2.819127 & 3.139112 & -4.893401 \\
\hline $\mathrm{C}$ & -4.166025 & 1.197705 & -0.093084 & $\mathrm{H}$ & -3.023591 & 4.057002 & -4.337572 \\
\hline $\mathrm{C}$ & -4.714692 & 1.444219 & -1.479439 & $\mathrm{H}$ & -2.399700 & 3.412651 & -5.866179 \\
\hline $\mathrm{C}$ & -6.034188 & 1.401593 & -1.947637 & $\mathrm{H}$ & -1.997201 & 2.622948 & -4.359370 \\
\hline $\mathrm{H}$ & -6.902851 & 1.168299 & -1.374092 & $\mathrm{C}$ & -5.128648 & 3.065406 & -5.866131 \\
\hline $\mathrm{C}$ & -6.012328 & 1.703658 & -3.337784 & $\mathrm{H}$ & -6.013868 & 2.454317 & -6.070288 \\
\hline $\mathrm{H}$ & -6.861758 & 1.735123 & -3.982208 & $\mathrm{H}$ & -4.712160 & 3.391027 & -6.823634 \\
\hline
\end{tabular}




\begin{tabular}{|c|c|c|c|}
\hline $\mathrm{H}$ & -5.450452 & 3.952557 & -5.312351 \\
\hline C & -1.703191 & -2.129873 & -5.946751 \\
\hline C & -2.064266 & -2.804235 & -4.642369 \\
\hline $\mathrm{C}$ & -3.026791 & -3.797645 & -4.399774 \\
\hline $\mathrm{H}$ & -3.665381 & -4.248372 & -5.126504 \\
\hline $\mathrm{C}$ & -3.021796 & -4.075503 & -3.008917 \\
\hline $\mathrm{H}$ & -3.654652 & -4.767480 & -2.496022 \\
\hline $\mathrm{C}$ & -2.052930 & -3.251044 & -2.419921 \\
\hline C & -1.699830 & -3.110143 & -0.962595 \\
\hline C & -1.616210 & -4.544462 & -0.401925 \\
\hline $\mathrm{C}$ & -2.754749 & -6.242366 & 0.902737 \\
\hline $\mathrm{H}$ & -2.189906 & -6.969728 & 0.290450 \\
\hline $\mathrm{H}$ & -3.827842 & -6.505930 & 0.933278 \\
\hline $\mathrm{C}$ & -2.166829 & -6.047963 & 2.272810 \\
\hline C & -1.245539 & -6.949645 & 2.809164 \\
\hline $\mathrm{H}$ & -0.966000 & -7.842793 & 2.251106 \\
\hline $\mathrm{C}$ & -0.645006 & -6.708111 & 4.050537 \\
\hline $\mathrm{H}$ & 0.079839 & -7.422405 & 4.433098 \\
\hline $\mathrm{C}$ & -0.946668 & -5.528665 & 4.751403 \\
\hline $\mathrm{C}$ & -1.911244 & -4.631277 & 4.229992 \\
\hline $\mathrm{H}$ & -2.157417 & -3.710548 & 4.757981 \\
\hline $\mathrm{C}$ & -2.506726 & -4.897095 & 3.006747 \\
\hline $\mathrm{H}$ & -3.218526 & -4.177205 & 2.567542 \\
\hline $\mathrm{C}$ & 1.064744 & -5.571256 & 6.303499 \\
\hline C & 233 & -6.882699 & 6.276336 \\
\hline $\mathrm{H}$ & 008 & -7.691704 & 5.957281 \\
\hline $\mathrm{C}$ & 2.855989 & -7.160281 & 6.630417 \\
\hline $\mathrm{H}$ & 3.212600 & -8.186750 & 6.58279 \\
\hline $\mathrm{C}$ & 3.708212 & -6.138552 & 7.028053 \\
\hline $\mathrm{H}$ & 4.741258 & -6.356242 & 7.29111 \\
\hline $\mathrm{C}$ & 3.253006 & -4.809603 & 7.09621 \\
\hline $\mathrm{H}$ & 3.935018 & -4.017992 & 7.41390 \\
\hline C & 1.942487 & -4.512735 & 6.738165 \\
\hline $\mathrm{C}$ & 467 & -3.173383 & 7.285919 \\
\hline $\mathrm{C}$ & -0.45 & -2.161369 & 8.217513 \\
\hline $\mathrm{C}$ & 358 & -1.120418 & 8.70392 \\
\hline $\mathrm{H}$ & 1.430749 & -1.089676 & 8.411416 \\
\hline $\mathrm{C}$ & -0.116320 & -0.143933 & 9.544577 \\
\hline $\mathrm{H}$ & 0.529578 & 0.656459 & 9.90094 \\
\hline $\mathrm{C}$ & -1.471942 & -0.172748 & 9.94071 \\
\hline $\mathrm{H}$ & -1.858073 & 0.614185 & 10.58515 \\
\hline $\mathrm{C}$ & -2.291967 & -1.195052 & 9.509091 \\
\hline $\mathrm{H}$ & -3.337853 & -1.222440 & 9.819937 \\
\hline $\mathrm{C}$ & -1.80 & -2.221839 & 8.655603 \\
\hline $\mathrm{C}$ & -2.65 & -3.33 & 8.264735 \\
\hline $\mathrm{C}$ & -3.991086 & -3.444538 & 8.748730 \\
\hline $\mathrm{H}$ & -4.388155 & -2.649209 & 9.381997 \\
\hline $\mathrm{C}$ & -4.780740 & -4.529818 & 8.428798 \\
\hline $\mathrm{H}$ & -5.801667 & -4.600384 & 8.797357 \\
\hline $\mathrm{C}$ & -4.264458 & -5.562879 & 7.613537 \\
\hline $\mathrm{H}$ & -4.890623 & -6.420586 & 7.375728 \\
\hline $\mathrm{C}$ & -2.979523 & -5.475491 & 7.118316 \\
\hline $\mathrm{H}$ & -2.583722 & -6.275008 & 6.488712 \\
\hline $\mathrm{C}$ & -2.160243 & -4.352084 & 7.414847 \\
\hline $\mathrm{C}$ & -0.820972 & -4.207015 & 6.880314 \\
\hline $\mathrm{C}$ & 2.015260 & -2.022066 & 6.30045 \\
\hline $\mathrm{C}$ & 3.346757 & -1.720858 & 6.623811 \\
\hline $\mathrm{H}$ & 3.918820 & -2.369347 & 7.28715 \\
\hline $\mathrm{C}$ & 3.951180 & -0.589064 & 6.06763 \\
\hline $\mathrm{H}$ & 4.992067 & -0.372555 & 6.30272 \\
\hline $\mathrm{C}$ & 3.234398 & 0.251825 & 5.20942 \\
\hline $\mathrm{C}$ & 1.892843 & -0.043143 & 4.90742 \\
\hline $\mathrm{H}$ & 1.346402 & 0.608566 & 4.2168 \\
\hline $\mathrm{C}$ & 1.281631 & -1.168955 & 5.44603 \\
\hline $\mathrm{H}$ & 0.248835 & -1.408129 & 5.18704 \\
\hline $\mathrm{C}$ & 3.897305 & 1.458256 & 4.61162 \\
\hline
\end{tabular}

\begin{tabular}{|c|c|c|c|}
\hline $\mathrm{H}$ & 3.748046 & 2.369374 & 5.224286 \\
\hline F & 4.976921 & 1.314589 & 4.422435 \\
\hline $\mathrm{C}$ & 3.485468 & 2.886654 & 2.699856 \\
\hline C & 2.879074 & 2.864773 & 1.283300 \\
\hline C & 3.947900 & 2.295980 & 0.385877 \\
\hline C & 5.342209 & 2.419124 & 0.463609 \\
\hline $\mathrm{H}$ & 5.888669 & 2.963039 & 1.204260 \\
\hline $\mathrm{C}$ & 5.895341 & 1.716759 & -0.639624 \\
\hline $\mathrm{H}$ & 6.934068 & 1.632809 & $-0.87022 \epsilon$ \\
\hline C & 4.833443 & 1.177629 & -1.382381 \\
\hline $\mathrm{C}$ & 4.850477 & 0.373898 & -2.663706 \\
\hline $\mathrm{C}$ & 4.862628 & 1.316958 & -3.845966 \\
\hline C & 5.743444 & 1.386188 & -4.929938 \\
\hline $\mathrm{H}$ & 6.606310 & 0.780301 & -5.089891 \\
\hline $\mathrm{C}$ & 5.284370 & 2.416756 & -5.797860 \\
\hline $\mathrm{H}$ & 5.748318 & 2.706865 & -6.713092 \\
\hline C & 4.127876 & 2.968174 & -5.235349 \\
\hline C & 3.220084 & 4.054823 & -5.768543 \\
\hline C & 3.008006 & 5.110259 & -4.707798 \\
\hline C & 3.779020 & 6.246530 & -4.427346 \\
\hline $\mathrm{H}$ & 4.668568 & 6.548852 & -4.932910 \\
\hline C & 3.180928 & 6.914626 & -3.3 \\
\hline $\mathrm{H}$ & 3.544294 & 7.803634 & -2.8 \\
\hline $\mathrm{C}$ & 2.051091 & 6.179275 & -2.943375 \\
\hline C & 1.092152 & 6.434304 & -1.8 \\
\hline C & 1.683874 & 5.859085 & -0.534331 \\
\hline C & 2.004100 & 6.474007 & 0.684737 \\
\hline $\mathrm{H}$ & 1.889845 & 7.506397 & 0.925923 \\
\hline C & 2.521186 & 5.474264 & 1.552237 \\
\hline $\mathrm{H}$ & 2.880017 & 5.626629 & 2.549097 \\
\hline C & 2.507171 & 4.261068 & 0.85510 \\
\hline C & 1.611078 & 1.99 & 1.2 \\
\hline $\mathrm{H}$ & 1.831179 & 0.964375 & 1.60395 \\
\hline 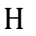 & 1.157964 & 1.943317 & 0.28308 \\
\hline$H$ & 0.847210 & 2.403592 & 1.958337 \\
\hline C & 3.616621 & -0.541004 & -2.767953 \\
\hline $\mathrm{H}$ & 3.543513 & -1.226230 & -1.919806 \\
\hline $\mathrm{H}$ & 3.641178 & -1.137646 & -3.685488 \\
\hline $\mathrm{H}$ & 2.659470 & 0.013561 & -2.813724 \\
\hline $\mathrm{C}$ & 6.121533 & -0.503885 & -2.673619 \\
\hline $\mathrm{H}$ & 6.154794 & -1.138908 & -1.782676 \\
\hline $\mathrm{H}$ & 7.026653 & 0.111867 & -2.6 \\
\hline $\mathrm{H}$ & 6.140776 & -1.151393 & -3.555200 \\
\hline $\mathrm{C}$ & 1.886631 & 3.420633 & -6.205916 \\
\hline$H$ & 1.353105 & 2.940138 & -5.365606 \\
\hline $\mathrm{H}$ & 2.045125 & 2.639812 & -6.954811 \\
\hline $\mathrm{H}$ & 1.202855 & 4.166218 & -6.61972 \\
\hline C & -0.278994 & 5.781283 & -2.063847 \\
\hline $\mathrm{H}$ & -0.764162 & 6.207880 & -2.946157 \\
\hline $\mathrm{H}$ & -0.946172 & 5.905206 & -1.206932 \\
\hline $\mathrm{H}$ & -0.198691 & 4.69 & -2.249473 \\
\hline $\mathrm{C}$ & 0.893186 & 7.957985 & -1.648209 \\
\hline$H$ & 0.164017 & 8.179103 & -0.86397 \\
\hline $\mathrm{H}$ & 0.536506 & 8.394598 & -2.586024 \\
\hline$H$ & 1.834568 & 8.452467 & -1.385570 \\
\hline $\mathrm{C}$ & -1.840865 & -3.164455 & -7.086896 \\
\hline $\mathrm{H}$ & -1.235200 & -4.050861 & -6.874554 \\
\hline $\mathrm{H}$ & -1.509234 & -2.741720 & -8.039371 \\
\hline $\mathrm{H}$ & -2.880999 & -3.486676 & -7.202393 \\
\hline C & -0.253833 & -1.609927 & -5.929064 \\
\hline $\mathrm{H}$ & -0.073460 & -0.887366 & -5.113075 \\
\hline 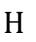 & -0.009032 & -1.088359 & -6.858691 \\
\hline 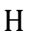 & 0.464410 & -2.421949 & -5.787468 \\
\hline $\mathrm{C}$ & -0.293216 & -2.520191 & -0.748743 \\
\hline $\mathrm{H}$ & -0.164991 & -1.553704 & -1.271027 \\
\hline $\mathrm{H}$ & 0.488840 & -3.185956 & -1.13853 \\
\hline
\end{tabular}




$\begin{array}{lrrr}\mathrm{H} & -0.091148 & -2.337520 & 0.311431 \\ \mathrm{C} & -5.229111 & 1.614758 & 0.945865 \\ \mathrm{H} & -6.129400 & 0.997836 & 0.854886 \\ \mathrm{H} & -5.521415 & 2.658885 & 0.797470 \\ \mathrm{H} & -4.845472 & 1.505940 & 1.964210 \\ \mathrm{C} & -2.886245 & 2.028463 & 0.143442 \\ \mathrm{H} & -2.486950 & 1.861918 & 1.147852 \\ \mathrm{H} & -3.074653 & 3.098393 & 0.021083 \\ \mathrm{H} & -2.084787 & 1.754254 & -0.563895 \\ \mathrm{~N} & -2.877132 & -0.900205 & -0.728167 \\ \mathrm{~N} & -3.893117 & 1.764332 & -2.563887 \\ \mathrm{~N} & -1.469215 & -2.475291 & -3.425071 \\ \mathrm{~N} & -0.263324 & -5.155562 & 5.946434 \\ \mathrm{~N} & 1.360328 & -3.201509 & 6.790899 \\ \mathrm{~N} & 3.646244 & 1.538829 & -0.748468 \\ \mathrm{~N} & 3.875463 & 2.293032 & -4.034854 \\ \mathrm{~N} & 1.951420 & 5.074231 & -3.793756 \\ \mathrm{~N} & 1.996705 & 4.504504 & -0.425762\end{array}$

$\begin{array}{lrcc}\mathrm{O} & -0.676891 & -5.304115 & -0.465459 \\ \mathrm{O} & -2.788199 & -4.961422 & 0.210601 \\ \mathrm{O} & 3.268336 & 1.657219 & 3.310621 \\ \mathrm{O} & 4.092467 & 3.740832 & 3.301736 \\ \mathrm{H} & -0.719397 & -1.765597 & -3.282501 \\ \mathrm{H} & -2.404690 & 0.105255 & -4.326502 \\ \mathrm{H} & -2.867973 & 1.853179 & -2.531611 \\ \mathrm{H} & -2.375983 & -0.468459 & -1.521908 \\ \mathrm{H} & 1.212032 & 4.342176 & -3.750638 \\ \mathrm{H} & 3.103376 & 2.478027 & -3.397331 \\ \mathrm{H} & 2.698473 & 1.300266 & -1.072754 \\ \mathrm{H} & 1.874314 & 3.793991 & -1.165907 \\ \mathrm{O} & 0.103733 & 2.930035 & -3.643223 \\ \mathrm{O} & 1.434559 & 2.237668 & -2.017311 \\ \mathrm{C} & -0.228093 & 0.681667 & -2.826092 \\ \mathrm{O} & -1.467146 & 0.678522 & -2.583149 \\ \mathrm{O} & 0.424410 & -0.354398 & -3.068034 \\ \mathrm{C} & 0.489284 & 2.061735 & -2.832924\end{array}$

Cartesian coordinates of the optimized complex DPAC-bisC4P $\supset \mathbf{C 4}^{2-}$ :

\begin{tabular}{|c|c|c|c|c|c|c|c|}
\hline Symbol & $\mathrm{X}$ & $Y$ & Z & & $\mathrm{X}$ & $Y$ & $Z$ \\
\hline $\bar{C}$ & -0.782995 & -3.503063 & -0.916353 & $\mathrm{H}$ & -3.768070 & -5.846312 & -1.934275 \\
\hline $\mathrm{C}$ & 0.281527 & -3.522697 & -0.002606 & $\mathrm{C}$ & -2.364804 & -4.325184 & -2.709850 \\
\hline $\mathrm{H}$ & 1.065277 & -4.249925 & 0.042246 & C & -1.055141 & -4.512923 & -2.000078 \\
\hline $\mathrm{C}$ & 2.773196 & 5.883287 & -6.541644 & C & -1.021176 & -5.883946 & -1.279543 \\
\hline $\mathrm{H}$ & 1.711670 & 6.076543 & -6.724991 & $\mathrm{C}$ & -2.091802 & -7.019343 & 0.602068 \\
\hline $\mathrm{H}$ & 3.226277 & 5.531947 & -7.472937 & $\mathrm{H}$ & -1.281461 & -7.747511 & 0.404867 \\
\hline $\mathrm{H}$ & 3.245176 & 6.834685 & -6.273344 & $\mathrm{H}$ & -3.080919 & -7.503968 & 0.511560 \\
\hline $\mathrm{C}$ & 0.135135 & -2.400270 & 0.848954 & C & -1.926776 & -6.305822 & 1.916401 \\
\hline $\mathrm{H}$ & 0.790480 & -2.132711 & 1.650343 & $\mathrm{C}$ & -1.599366 & -7.003280 & 3.079663 \\
\hline $\mathrm{C}$ & -1.013659 & -1.701714 & 0.446058 & $\mathrm{H}$ & -1.467155 & -8.082966 & 3.053507 \\
\hline $\mathrm{C}$ & -1.554088 & -0.426379 & 1.052202 & C & -1.425514 & -6.327736 & 4.294885 \\
\hline $\mathrm{C}$ & -2.906417 & -0.048370 & 0.497614 & $\mathrm{H}$ & -1.172173 & -6.900492 & 5.183999 \\
\hline $\mathrm{C}$ & -4.170864 & -0.099363 & 1.097033 & C & -1.554469 & -4.930423 & 4.340574 \\
\hline $\mathrm{H}$ & -4.401131 & -0.487073 & 2.065473 & $\mathrm{C}$ & -1.898172 & -4.223020 & 3.163835 \\
\hline $\mathrm{C}$ & -5.109298 & 0.451201 & 0.181872 & $\mathrm{H}$ & -1.982241 & -3.126912 & 3.162687 \\
\hline $\mathrm{H}$ & -6.159748 & 0.543084 & 0.345103 & $\mathrm{C}$ & -2.092409 & -4.908703 & 1.975332 \\
\hline $\mathrm{C}$ & -4.410710 & 0.832846 & -0.970943 & $\mathrm{H}$ & -2.349894 & -4.358696 & 1.059153 \\
\hline $\mathrm{N}$ & -4.419057 & -0.517375 & -3.723425 & C & -0.424673 & -4.547959 & 6.588073 \\
\hline $\mathrm{C}$ & -5.342094 & 0.400158 & -3.214430 & $\mathrm{C}$ & -0.372959 & -5.811292 & 7.166110 \\
\hline $\mathrm{C}$ & -6.590244 & 0.112391 & -3.780263 & $\mathrm{H}$ & -1.028559 & -6.608303 & 6.808262 \\
\hline $\mathrm{H}$ & -7.506335 & 0.626387 & -3.598329 & C & 0.545827 & -6.073214 & 8.198507 \\
\hline $\mathrm{C}$ & -6.425662 & -1.005595 & -4.645716 & $\mathrm{H}$ & 0.589525 & -7.072244 & 8.625541 \\
\hline $\mathrm{H}$ & -7.197732 & -1.466055 & -5.218743 & $\mathrm{C}$ & 1.384925 & -5.071372 & 8.665207 \\
\hline C & -5.079352 & -1.386053 & -4.596556 & $\mathrm{H}$ & 2.096689 & -5.275413 & 9.461635 \\
\hline $\mathrm{C}$ & -4.928790 & 1.477628 & -2.238602 & C & 1.326777 & -3.777190 & 8.117130 \\
\hline $\mathrm{C}$ & -3.845100 & 2.360396 & -2.889980 & $\mathrm{H}$ & 1.989515 & -2.997821 & 8.500640 \\
\hline $\mathrm{H}$ & -3.510228 & 3.149184 & -2.211502 & C & 0.441895 & -3.502540 & 7.080492 \\
\hline $\mathrm{H}$ & -4.216236 & 2.825843 & -3.806917 & $\mathrm{C}$ & -1.078893 & -1.862158 & 6.182487 \\
\hline $\mathrm{H}$ & -2.955434 & 1.768811 & -3.177139 & C & -1.586197 & -0.521544 & 6.418345 \\
\hline $\mathrm{C}$ & -6.142218 & 2.365113 & -1.885019 & $\mathrm{C}$ & -0.797466 & 0.505695 & 7.002567 \\
\hline $\mathrm{H}$ & -6.962408 & 1.766271 & -1.475178 & $\mathrm{H}$ & 0.211824 & 0.279459 & 7.350482 \\
\hline $\mathrm{H}$ & -6.515137 & 2.885569 & -2.771764 & C & -1.289138 & 1.788939 & 7.135059 \\
\hline $\mathrm{H}$ & -5.866107 & 3.113742 & -1.136079 & $\mathrm{H}$ & -0.672038 & 2.574197 & 7.567745 \\
\hline $\mathrm{C}$ & -4.345508 & -2.513655 & -5.284914 & C & -2.595605 & 2.095524 & 6.693289 \\
\hline $\mathrm{C}$ & -3.813292 & -3.445813 & -4.217983 & $\mathrm{H}$ & -2.960924 & 3.117586 & 6.770459 \\
\hline $\mathrm{C}$ & -4.470740 & -4.495489 & -3.555882 & $\mathrm{C}$ & -3.393970 & 1.101317 & 6.165614 \\
\hline $\mathrm{H}$ & -5.476421 & -4.813266 & -3.717886 & $\mathrm{H}$ & -4.405534 & 1.335652 & 5.827524 \\
\hline $\mathrm{C}$ & -3.564869 & -5.043306 & -2.612816 & C & -2.920506 & -0.233168 & 6.039994 \\
\hline
\end{tabular}




\begin{tabular}{|c|c|c|c|}
\hline $\mathrm{C}$ & -3.799241 & -1.298218 & 5.572037 \\
\hline $\mathrm{C}$ & -5.154418 & -1.035147 & 5.232432 \\
\hline $\mathrm{H}$ & -5.511584 & -0.003432 & 5.247395 \\
\hline C & -6.011240 & -2.056019 & 4.874620 \\
\hline $\mathrm{H}$ & -7.041797 & -1.842821 & 4.59709 \\
\hline C & -5.549469 & -3.391576 & 4.858525 \\
\hline $\mathrm{H}$ & -6.236764 & -4.192266 & 4.59197 \\
\hline $\mathrm{C}$ & -4.231822 & -3.671049 & 5.160049 \\
\hline $\mathrm{H}$ & -3.880321 & -4.704226 & 5.14309 \\
\hline $\mathrm{C}$ & -3.322435 & -2.628362 & 5.484562 \\
\hline $\mathrm{C}$ & -1.910750 & -2.870410 & 5.722231 \\
\hline $\mathrm{C}$ & 1.375711 & -1.381461 & 6.04466 \\
\hline $\mathrm{C}$ & 2.546158 & -1.234681 & 6.80521 \\
\hline $\mathrm{H}$ & 2.657593 & -1.739578 & 7.76400 \\
\hline $\mathrm{C}$ & 3.593537 & -0.442710 & 6.32076 \\
\hline $\mathrm{H}$ & 4.505566 & -0.354077 & 6.90788 \\
\hline $\mathrm{C}$ & 3.476355 & 0.223041 & 5.09824 \\
\hline $\mathrm{C}$ & 2.287068 & 0.101457 & 4.35478 \\
\hline $\mathrm{H}$ & 2.198458 & 0.627499 & 3.39513 \\
\hline $\mathrm{C}$ & 1.249579 & -0.696966 & 4.81322 \\
\hline $\mathrm{H}$ & 0.340311 & -0.807873 & 4.20234 \\
\hline $\mathrm{C}$ & 4.616319 & 1.045088 & 4.56610 \\
\hline $\mathrm{H}$ & 4.635047 & 2.070385 & 4.9864 \\
\hline $\mathrm{H}$ & 5.599674 & 0.564254 & 4.7106 \\
\hline $\mathrm{C}$ & 4.771749 & 2.274623 & 2.46143 \\
\hline $\mathrm{C}$ & 4.281258 & 2.176359 & 0.99785 \\
\hline $\mathrm{C}$ & 5.344382 & 2.754141 & 0.10548 \\
\hline $\mathrm{C}$ & 6.231920 & 3.813301 & 0.34407 \\
\hline $\mathrm{H}$ & 6.300899 & 4.379821 & 1.25174 \\
\hline $\mathrm{C}$ & 7.006959 & 3.996468 & -0.82867 \\
\hline $\mathrm{H}$ & 7.763286 & 4.735640 & -0.97061 \\
\hline $\mathrm{C}$ & 6.579633 & 3.049946 & -1.77458 \\
\hline $\mathrm{C}$ & 7.044807 & 2.836217 & -3.19782 \\
\hline $\mathrm{C}$ & 6.251546 & 3.734865 & -4.12007 \\
\hline $\mathrm{C}$ & 6.694068 & 4.662745 & -5.07061 \\
\hline $\mathrm{H}$ & 7.706229 & 4.913399 & -5.29287 \\
\hline $\mathrm{C}$ & 5.546052 & 5.226594 & -5.69421 \\
\hline $\mathrm{H}$ & 5.557503 & 5.969391 & -6.45906 \\
\hline $\mathrm{C}$ & 4.415737 & 4.635720 & -5.11826 \\
\hline $\mathrm{C}$ & 2.947925 & 4.841620 & -5.41542 \\
\hline $\mathrm{C}$ & 2.253725 & 5.349273 & -4.17180 \\
\hline $\mathrm{C}$ & 2.107191 & 6.657415 & -3.69373 \\
\hline $\mathrm{H}$ & 2.483011 & 7.545133 & -4.15128 \\
\hline $\mathrm{C}$ & 1.383960 & 6.594190 & -2.47022 \\
\hline $\mathrm{H}$ & 1.125261 & 7.428873 & -1.85736 \\
\hline $\mathrm{C}$ & 1.093520 & 5.248834 & -2.21185 \\
\hline $\mathrm{C}$ & 0.321093 & 4.617970 & -1.07709 \\
\hline $\mathrm{C}$ & 1.248754 & 4.077746 & -0.01037 \\
\hline $\mathrm{C}$ & 1.107436 & 4.145358 & 1.3833 \\
\hline $\mathrm{H}$ & 0.319041 & 4.625081 & 1.9200 \\
\hline $\mathrm{C}$ & 2.204295 & 3.459815 & 1.9675 \\
\hline $\mathrm{H}$ & 2.382213 & 3.344925 & 3.0146 \\
\hline $\mathrm{C}$ & 3.006197 & 2.981434 & 0.9230 \\
\hline $\mathrm{C}$ & 3.992378 & 0.706489 & 0.65940 \\
\hline $\mathrm{H}$ & 4.882896 & 0.079975 & 0.7819 \\
\hline $\mathrm{H}$ & 3.657704 & 0.579456 & -0.38491 \\
\hline $\mathrm{H}$ & 3.202554 & 0.292293 & 1.2982 \\
\hline $\mathrm{C}$ & 6.848158 & 1.371686 & -3.63496 \\
\hline $\mathrm{H}$ & 7.431113 & 0.686025 & -3.01497 \\
\hline $\mathrm{H}$ & 7.134173 & 1.228871 & -4.67960 \\
\hline $\mathrm{H}$ & 5.792123 & 1.048456 & -3.54880 \\
\hline $\mathrm{C}$ & 8.547450 & 3.183109 & -3.28474 \\
\hline $\mathrm{H}$ & 9.118536 & 2.596682 & -2.55854 \\
\hline $\mathrm{H}$ & 8.718108 & 4.243243 & -3.0682 \\
\hline $\mathrm{H}$ & 8.940336 & 2.973539 & -4.2831 \\
\hline
\end{tabular}

\begin{tabular}{|c|c|c|c|}
\hline C & 2.340783 & 3.501424 & -5.881468 \\
\hline $\mathrm{H}$ & 2.451227 & 2.715698 & -5.112764 \\
\hline H & 2.847174 & 3.132407 & -6.777659 \\
\hline $\mathrm{H}$ & 1.274926 & 3.598445 & -6.102149 \\
\hline $\mathrm{C}$ & -0.552194 & 3.460912 & -1.603354 \\
\hline $\mathrm{H}$ & -1.198768 & 3.790614 & -2.422616 \\
\hline $\mathrm{H}$ & -1.191570 & 3.054648 & -0.811361 \\
\hline $\mathrm{H}$ & 0.054657 & 2.616380 & -1.982083 \\
\hline C & -0.603345 & 5.690442 & -0.457695 \\
\hline $\mathrm{H}$ & -1.271512 & 5.251811 & 0.289682 \\
\hline 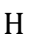 & -1.221878 & 6.158778 & -1.229941 \\
\hline 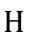 & -0.017822 & 6.476205 & 0.031917 \\
\hline C & -5.310830 & -3.297223 & -6.200201 \\
\hline $\mathrm{H}$ & -4.803421 & -4.157599 & -6.646922 \\
\hline $\mathrm{H}$ & -5.686740 & -2.659766 & -7.005253 \\
\hline $\mathrm{H}$ & -6.171450 & -3.672376 & -5.636102 \\
\hline C & -3.204681 & -1.939540 & -6.146503 \\
\hline $\mathrm{H}$ & -2.438058 & -1.425094 & -5.537763 \\
\hline $\mathrm{H}$ & -3.582294 & -1.205687 & -6.862772 \\
\hline 4 & -2.681376 & -2.727316 & -6.695222 \\
\hline C & 0.100598 & -4.483700 & -3.014590 \\
\hline $\mathrm{H}$ & 0.214996 & -3.464123 & -3.436779 \\
\hline $\mathrm{H}$ & -0.074429 & -5.169287 & -3.848587 \\
\hline $\mathrm{H}$ & 1.057727 & -4.745177 & -2.549661 \\
\hline C & -1.676726 & -0.631105 & 2.578612 \\
\hline $\mathrm{H}$ & -2.244294 & -1.54 & 2.812838 \\
\hline $\mathrm{H}$ & -2.181612 & 164 & 0756 \\
\hline $\mathrm{H}$ & -0.68 & -0.7 & 3.048850 \\
\hline 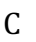 & -0.53 & 536 & 6130 \\
\hline 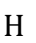 & 0.41 & 3610 & 4658 \\
\hline $\mathrm{H}$ & -0.905399 & 1.673618 & 1.107547 \\
\hline $\mathrm{H}$ & -0.313275 & 0.778556 & -0.305374 \\
\hline $\mathrm{N}$ & -1.567210 & -2.378179 & -0.643108 \\
\hline $\mathrm{N}$ & -3.065864 & 0.505946 & -0.778632 \\
\hline $\mathrm{N}$ & -2.529170 & -3.339913 & -3.690546 \\
\hline $\mathrm{N}$ & -1.317167 & -4.171721 & 5.530047 \\
\hline $\mathrm{N}$ & 0.2 & -2.2 & 966 \\
\hline $\mathrm{N}$ & 062 & 436 & 8087 \\
\hline $\mathrm{N}$ & 4.853402 & 3.721138 & 3577 \\
\hline $\mathrm{N}$ & 1.63 & 4.493292 & -3.25516 \\
\hline $\mathrm{N}$ & 2.41 & 3.352397 & -0.286666 \\
\hline 0 & -0.191737 & -6.760543 & -1.337225 \\
\hline 0 & -2.120936 & -6.013951 & -0.448077 \\
\hline 0 & 4.412491 & 1.110907 & 3.126601 \\
\hline 0 & 5.350143 & 3.148827 & 3.060380 \\
\hline H & -1.796270 & -2.635043 & -3.973231 \\
\hline H & -3.40 & -0.5 & -3.4 \\
\hline H & -2.314665 & 0.6 & -1.4 \\
\hline $\mathrm{H}$ & -2.391941 & -2.102491 & -1.158473 \\
\hline $\mathrm{H}$ & 1.606409 & 3.469147 & -3.33140 \\
\hline $\mathrm{H}$ & 4.238662 & 3.136669 & -3.566922 \\
\hline $\mathrm{H}$ & 5.042737 & 1.514808 & -1.668734 \\
\hline $\mathrm{H}$ & 2.773469 & 3.124078 & -1.217717 \\
\hline 0 & 2.763230 & 2.094521 & $-2.94742 \mathrm{c}$ \\
\hline 0 & 4.037390 & 0.357028 & -2.506501 \\
\hline $\mathrm{C}$ & -0.667531 & -0.401427 & -3.64 \\
\hline 0 & -1.746469 & -0.0 & -3.0 \\
\hline 0 & -0.563085 & -1.492377 & -4.255826 \\
\hline C & 2.928910 & 0.841459 & -2.84983 \\
\hline C & 0.510795 & 0.562351 & -3.58066 \\
\hline $\mathrm{H}$ & 0.260967 & 1.417302 & -2.90854 \\
\hline $\mathrm{H}$ & 0.666116 & 1.001998 & -4.58448 \\
\hline c & 1.781912 & -0.121799 & -3.100300 \\
\hline & 2.101563 & -0.897404 & -3.82730 \\
\hline & 1.591406 & -0.687149 & -2.16219 \\
\hline
\end{tabular}




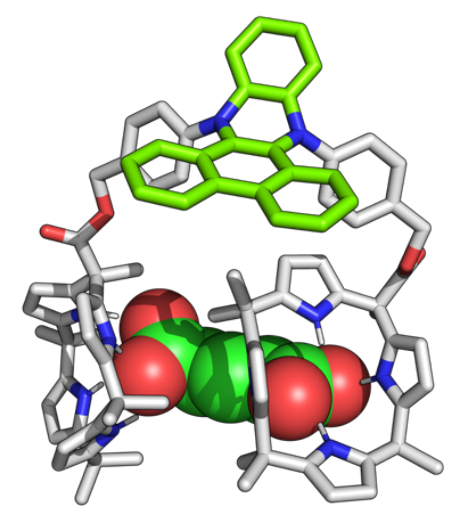

Figure S29. Optimized structure for DPAC-bisC4P $\supset \mathrm{C5}^{2-}$.

Cartesian coordinates of the optimized complex DPAC-bisC4PつC $\mathbf{5}^{\mathbf{2}}$ :

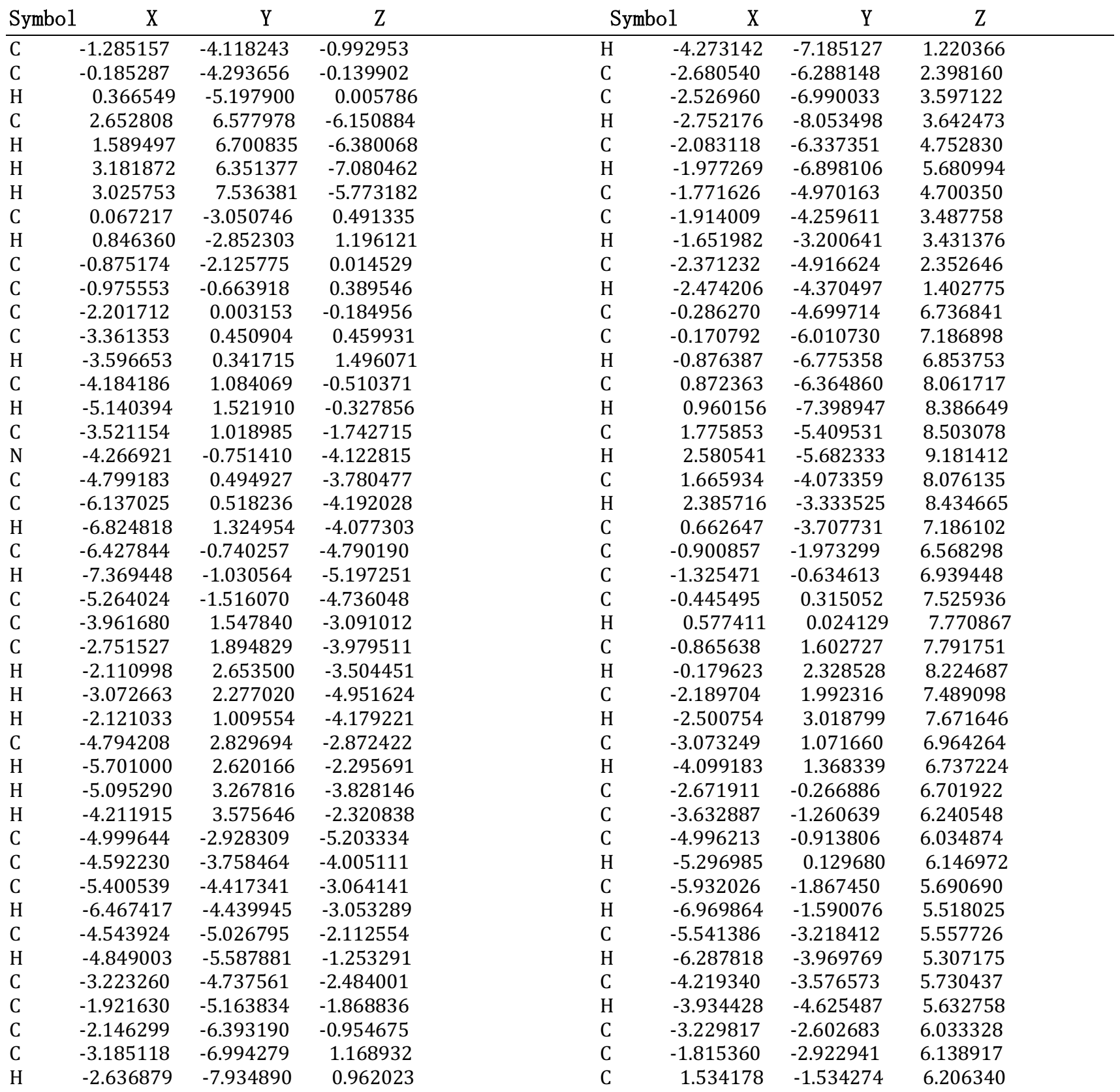




\begin{tabular}{|c|c|c|c|}
\hline $\mathrm{C}$ & 2.767076 & -1.436081 & 6.870181 \\
\hline $\mathrm{H}$ & 2.958368 & -2.001472 & 7.781980 \\
\hline $\mathrm{C}$ & 3.769678 & -0.609671 & 6.352708 \\
\hline $\mathrm{H}$ & 4.727361 & -0.553884 & 6.86697 \\
\hline C & 3.550409 & 0.136866 & 5.19129 \\
\hline $\mathrm{C}$ & 2.307311 & 0.051236 & 4.53826 \\
\hline $\mathrm{H}$ & 2.142496 & 0.632653 & 3.6227 \\
\hline $\mathrm{C}$ & 1.308015 & -0.776813 & 5.03243 \\
\hline $\mathrm{H}$ & 0.353378 & -0.849637 & 4.5065 \\
\hline $\mathrm{C}$ & 4.642201 & 1.016889 & 4.6526 \\
\hline $\mathrm{H}$ & 4.684050 & 1.996409 & 5.1730 \\
\hline $\mathrm{H}$ & 5.635285 & 0.533611 & 4.6829 \\
\hline $\mathrm{C}$ & 4.530653 & 2.499133 & 2.7210 \\
\hline $\mathrm{C}$ & 4.113482 & 2.502561 & 1.23483 \\
\hline $\mathrm{C}$ & 5.182731 & 3.217091 & 0.45281 \\
\hline $\mathrm{C}$ & 5.971872 & 4.317068 & 0.81627 \\
\hline $\mathrm{H}$ & 5.958151 & 4.810448 & 1.7678 \\
\hline $\mathrm{C}$ & 6.770736 & 4.658271 & -0.30436 \\
\hline $\mathrm{H}$ & 7.468785 & 5.464028 & -0.35237 \\
\hline $\mathrm{C}$ & 6.456648 & 3.764986 & -1.34194 \\
\hline $\mathrm{C}$ & 7.005320 & 3.703174 & -2.74881 \\
\hline $\mathrm{C}$ & 6.171472 & 4.568094 & -3.66836 \\
\hline $\mathrm{C}$ & 6.584401 & 5.533362 & -4.59407 \\
\hline $\mathrm{H}$ & 7.585363 & 5.852065 & -4.77835 \\
\hline $\mathrm{C}$ & 5.425662 & 6.027926 & -5.25536 \\
\hline $\mathrm{H}$ & 5.417687 & 6.777554 & -6.01405 \\
\hline $\mathrm{C}$ & 4.315923 & 5.359427 & -4.72818 \\
\hline $\mathrm{C}$ & 2.855490 & 5.458113 & -5.10780 \\
\hline $\mathrm{C}$ & 2.025708 & 5.777283 & -3.88677 \\
\hline $\mathrm{C}$ & 1.682413 & 7.011164 & -3.32114 \\
\hline $\mathrm{H}$ & 1.971157 & 7.974546 & -3.67768 \\
\hline $\mathrm{C}$ & 0.893050 & 6.755201 & -2.16464 \\
\hline $\mathrm{H}$ & 0.489593 & 7.494363 & -1.50901 \\
\hline $\mathrm{C}$ & 0.761732 & 5.367435 & -2.03398 \\
\hline $\mathrm{C}$ & 0.044536 & 4.540884 & -0.99151 \\
\hline $\mathrm{C}$ & 0.998586 & 4.187917 & 0.1292 \\
\hline $\mathrm{C}$ & 0.894569 & 4.423999 & $1.5061 \epsilon$ \\
\hline $\mathrm{H}$ & 0.107560 & 4.944860 & 2.0049 \\
\hline $\mathrm{C}$ & 2.029155 & 3.844081 & 2.1349 \\
\hline $\mathrm{H}$ & 2.245889 & 3.871162 & 3.1810 \\
\hline $\mathrm{C}$ & 2.812096 & 3.258790 & 1.13337 \\
\hline $\mathrm{C}$ & 3.923150 & 1.056438 & 0.75462 \\
\hline $\mathrm{H}$ & 4.835005 & 0.462814 & 0.8869 \\
\hline $\mathrm{H}$ & 3.667796 & 1.003672 & -0.32014 \\
\hline $\mathrm{H}$ & 3.116740 & 0.555296 & 1.3030 \\
\hline $\mathrm{C}$ & 6.998545 & 2.258028 & -3.28524 \\
\hline $\mathrm{H}$ & 7.619120 & 1.601182 & -2.6694 \\
\hline $\mathrm{H}$ & 7.360207 & 2.216566 & -4.31523 \\
\hline $\mathrm{H}$ & 5.984644 & 1.817793 & -3.28406 \\
\hline $\mathrm{C}$ & 8.463571 & 4.215092 & -2.72988 \\
\hline $\mathrm{H}$ & 9.054900 & 3.659590 & -1.9951 \\
\hline $\mathrm{H}$ & 8.502574 & 5.275869 & -2.45861 \\
\hline $\mathrm{H}$ & 8.934215 & 4.098235 & -3.7095 \\
\hline $\mathrm{C}$ & 2.422650 & 4.112785 & -5.73159 \\
\hline $\mathrm{H}$ & 2.549578 & 3.277664 & -5.0227 \\
\hline $\mathrm{H}$ & 3.027332 & 3.876885 & -6.61147 \\
\hline $\mathrm{H}$ & 1.371009 & 4.131749 & -6.03013 \\
\hline $\mathrm{C}$ & -0.487590 & 3.229574 & -1.60124 \\
\hline $\mathrm{H}$ & -1.185313 & 3.412535 & -2.43245 \\
\hline $\mathrm{H}$ & -1.032598 & 2.641130 & -0.84930 \\
\hline $\mathrm{H}$ & 0.321205 & 2.589149 & -1.98282 \\
\hline
\end{tabular}

\begin{tabular}{|c|c|c|c|}
\hline $\mathrm{C}$ & -1.144421 & 5.353623 & -0.439609 \\
\hline $\mathrm{H}$ & -1.731346 & 4.755888 & 0.266493 \\
\hline $\mathrm{H}$ & -1.809323 & 5.663206 & -1.252246 \\
\hline $\mathrm{H}$ & -0.802315 & 6.254594 & 0.079615 \\
\hline $\mathrm{C}$ & -6.279142 & -3.534158 & -5.819201 \\
\hline $\mathrm{H}$ & -6.116432 & -4.580922 & -6.092764 \\
\hline $\mathrm{H}$ & -6.577892 & -2.983603 & -6.715650 \\
\hline $\mathrm{H}$ & -7.111999 & -3.499315 & -5.108372 \\
\hline $\mathrm{C}$ & -3.892038 & -2.915456 & -6.275140 \\
\hline $\mathrm{H}$ & -2.925811 & -2.555769 & -5.873367 \\
\hline $\mathrm{H}$ & -4.154235 & -2.252498 & -7.103420 \\
\hline $\mathrm{H}$ & -3.706907 & -3.916837 & -6.672208 \\
\hline $\mathrm{C}$ & -0.923278 & -5.579897 & -2.962722 \\
\hline $\mathrm{H}$ & -0.637065 & -4.702543 & -3.579067 \\
\hline $\mathrm{H}$ & -1.350099 & -6.329478 & -3.635712 \\
\hline $\mathrm{H}$ & -0.000100 & -5.986337 & -2.536467 \\
\hline $\mathrm{C}$ & -1.027830 & -0.565907 & 1.931695 \\
\hline $\mathrm{H}$ & -1.896167 & -1.098949 & 2.331496 \\
\hline $\mathrm{H}$ & -1.093840 & 0.481546 & 2.248562 \\
\hline $\mathrm{H}$ & -0.128303 & -0.995669 & 2.384185 \\
\hline $\mathrm{C}$ & 0.297398 & 0.043921 & -0.115401 \\
\hline $\mathrm{H}$ & 1.195204 & -0.348765 & 0.374329 \\
\hline $\mathrm{H}$ & 0.262022 & 1.123099 & 0.079885 \\
\hline $\mathrm{H}$ & 0.449473 & -0.100640 & -1.200185 \\
\hline $\mathrm{N}$ & -1.698586 & -2.786120 & -0.898895 \\
\hline $\mathrm{N}$ & -2.316656 & 0.340055 & -1.539978 \\
\hline $\mathrm{N}$ & -3.262839 & -3.949018 & -3.639374 \\
\hline $\mathrm{N}$ & -1.312005 & -4.240001 & 5.849702 \\
\hline $\mathrm{N}$ & 0.477272 & -2.382578 & 6.670840 \\
\hline $\mathrm{N}$ & 5.483507 & 2.885407 & -0.871901 \\
\hline $\mathrm{N}$ & 4.777511 & 4.467318 & -3.751366 \\
\hline $\mathrm{N}$ & 1.460084 & 4.777479 & -3.090622 \\
\hline $\mathrm{N}$ & 2.179078 & 3.472557 & -0.095672 \\
\hline 0 & -1.633537 & -7.487065 & -0.999226 \\
\hline 0 & -3.057160 & -6.087257 & 0.040939 \\
\hline 0 & 4.367894 & 1.221155 & 3.238399 \\
\hline 0 & 4.939265 & 3.383463 & 3.435687 \\
\hline $\mathrm{H}$ & -2.419563 & -3.561478 & -4.141959 \\
\hline $\mathrm{H}$ & -3.291315 & -1.052568 & -3.950146 \\
\hline $\mathrm{H}$ & -1.630436 & 0.114415 & -2.271040 \\
\hline $\mathrm{H}$ & -2.463910 & -2.374517 & -1.416007 \\
\hline $\mathrm{H}$ & 1.553362 & 3.768371 & -3.251077 \\
\hline $\mathrm{H}$ & 4.185341 & 3.849299 & -3.192966 \\
\hline $\mathrm{H}$ & 5.042609 & 2.104814 & -1.416408 \\
\hline $\mathrm{H}$ & 2.527611 & 3.156523 & -1.010106 \\
\hline $\mathrm{C}$ & 2.620947 & 0.326685 & -4.024843 \\
\hline $\mathrm{H}$ & 3.270175 & -0.571502 & -4.077600 \\
\hline $\mathrm{H}$ & 2.671858 & 0.805054 & -5.019813 \\
\hline $\mathrm{C}$ & 1.179844 & -0.082822 & -3.707469 \\
\hline $\mathrm{H}$ & 0.483940 & 0.749061 & -3.933265 \\
\hline $\mathrm{H}$ & 1.060843 & -0.280579 & -2.614915 \\
\hline $\mathrm{C}$ & 0.785698 & -1.330800 & -4.499701 \\
\hline $\mathrm{H}$ & 1.434133 & -2.185671 & -4.220827 \\
\hline $\mathrm{H}$ & 0.951930 & -1.174972 & -5.582680 \\
\hline $\mathrm{C}$ & 3.219920 & 1.275116 & -2.994488 \\
\hline $\mathrm{C}$ & -0.663324 & -1.758632 & -4.309275 \\
\hline 0 & -1.489295 & -0.994436 & -3.744278 \\
\hline 0 & 4.258059 & 0.903857 & -2.391368 \\
\hline 0 & 2.711948 & 2.416062 & -2.801112 \\
\hline 0 & -0.987540 & -2.889544 & -4.762919 \\
\hline
\end{tabular}




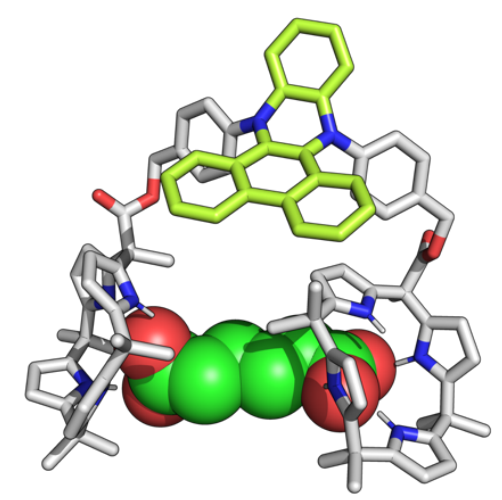

Figure S30. Optimized structure for DPAC-bisC4P $\supset \mathbf{C 6}^{2-}$.

Cartesian coordinates of the optimized complex DPAC-bisC4P $\supset \mathbf{C 6}^{2-}$ :

\begin{tabular}{|c|c|c|c|c|c|c|c|}
\hline Symbol & $\mathrm{X}$ & $Y$ & Z & & $\mathrm{X}$ & $Y$ & $Z$ \\
\hline $\mathrm{C}$ & -1.881976 & -4.193881 & -0.809474 & $\mathrm{H}$ & -3.026609 & -7.479688 & 4.527823 \\
\hline $\mathrm{C}$ & -0.664933 & -3.850190 & -0.204825 & $\mathrm{C}$ & -2.162904 & -5.671461 & 5.307054 \\
\hline $\mathrm{H}$ & 0.189050 & -4.484576 & -0.092520 & $\mathrm{H}$ & -2.058788 & -6.085860 & 6.308468 \\
\hline $\mathrm{C}$ & 3.252370 & 8.120059 & -4.610518 & $\mathrm{C}$ & -1.738803 & -4.365574 & 5.016215 \\
\hline $\mathrm{H}$ & 2.265885 & 8.444722 & -4.952535 & $\mathrm{C}$ & -1.893224 & -3.856054 & 3.704199 \\
\hline $\mathrm{H}$ & 3.932180 & 8.124608 & -5.468156 & $\mathrm{H}$ & -1.550736 & -2.850134 & 3.452832 \\
\hline $\mathrm{H}$ & 3.619064 & 8.856390 & -3.887127 & $\mathrm{C}$ & -2.467736 & -4.643696 & 2.718336 \\
\hline $\mathrm{C}$ & -0.767207 & -2.505107 & 0.231034 & $\mathrm{H}$ & -2.588622 & -4.246610 & 1.700933 \\
\hline $\mathrm{H}$ & -0.001789 & -1.947147 & 0.726295 & $\mathrm{C}$ & -0.268792 & -3.940940 & 7.047999 \\
\hline $\mathrm{C}$ & -2.044049 & -2.036125 & -0.113836 & $\mathrm{C}$ & -0.507203 & -5.040243 & 7.865071 \\
\hline $\mathrm{C}$ & -2.631840 & -0.679818 & 0.203126 & $\mathrm{H}$ & -1.399499 & -5.653613 & 7.719008 \\
\hline $\mathrm{C}$ & -3.883981 & -0.378740 & -0.582007 & $\mathrm{C}$ & 0.415064 & -5.380341 & 8.871327 \\
\hline $\mathrm{C}$ & -5.160134 & -0.015299 & -0.134809 & $\mathrm{H}$ & 0.224206 & -6.254860 & 9.488717 \\
\hline $\mathrm{H}$ & -5.492903 & 0.024582 & 0.878732 & $\mathrm{C}$ & 1.551652 & -4.610837 & 9.073766 \\
\hline $\mathrm{C}$ & -5.948493 & 0.293507 & -1.276350 & $\mathrm{H}$ & 2.264175 & -4.874100 & 9.851995 \\
\hline $\mathrm{H}$ & -6.971467 & 0.597359 & -1.264511 & $\mathrm{C}$ & 1.798537 & -3.477769 & 8.277462 \\
\hline $\mathrm{C}$ & -5.145930 & 0.117346 & -2.411640 & $\mathrm{H}$ & 2.694762 & -2.878565 & 8.455017 \\
\hline $\mathrm{N}$ & -5.175182 & -2.102046 & -4.555290 & $\mathrm{C}$ & 0.912338 & -3.137092 & 7.261638 \\
\hline $\mathrm{C}$ & -5.999046 & -0.978646 & -4.443257 & $\mathrm{C}$ & -0.154718 & -1.305922 & 6.098745 \\
\hline $\mathrm{C}$ & -7.267119 & -1.325555 & -4.925722 & $\mathrm{C}$ & -0.214989 & 0.144843 & 6.030255 \\
\hline $\mathrm{H}$ & -8.124293 & -0.693334 & -4.977236 & $\mathrm{C}$ & 0.899121 & 0.976648 & 6.321760 \\
\hline $\mathrm{C}$ & -7.218071 & -2.688620 & -5.331313 & $\mathrm{H}$ & 1.825826 & 0.533453 & 6.689594 \\
\hline $\mathrm{H}$ & -8.031681 & -3.245343 & -5.737346 & $\mathrm{C}$ & 0.830919 & 2.344108 & 6.142734 \\
\hline $\mathrm{C}$ & -5.920832 & -3.156337 & -5.090949 & $\mathrm{H}$ & 1.700280 & 2.971272 & 6.339077 \\
\hline $\mathrm{C}$ & -5.490388 & 0.324502 & -3.871390 & $\mathrm{C}$ & -0.364162 & 2.938693 & 5.680802 \\
\hline $\mathrm{C}$ & -4.256437 & 0.793085 & -4.667493 & $\mathrm{H}$ & -0.394909 & 4.013509 & 5.508084 \\
\hline $\mathrm{H}$ & -3.848299 & 1.722654 & -4.262997 & $\mathrm{C}$ & -1.475826 & 2.154778 & 5.447581 \\
\hline $\mathrm{H}$ & -4.504610 & 0.951844 & -5.720177 & $\mathrm{H}$ & -2.403447 & 2.614587 & 5.100832 \\
\hline $\mathrm{H}$ & -3.443203 & 0.043322 & -4.647743 & $\mathrm{C}$ & -1.436119 & 0.746785 & 5.638334 \\
\hline $\mathrm{C}$ & -6.583716 & 1.410111 & -3.977415 & $\mathrm{C}$ & -2.636318 & -0.063924 & 5.471286 \\
\hline $\mathrm{H}$ & -7.499967 & 1.100881 & -3.463410 & $\mathrm{C}$ & -3.882851 & 0.532371 & 5.137199 \\
\hline $\mathrm{H}$ & -6.834912 & 1.608201 & -5.023464 & $\mathrm{H}$ & -3.916962 & 1.604822 & 4.933703 \\
\hline $\mathrm{H}$ & -6.243258 & 2.345284 & -3.521854 & $\mathrm{C}$ & -5.036526 & -0.220856 & 5.059992 \\
\hline $\mathrm{C}$ & -5.312106 & -4.523045 & -5.300846 & $\mathrm{H}$ & -5.983170 & 0.241434 & 4.786618 \\
\hline $\mathrm{C}$ & -4.902310 & -5.066302 & -3.948808 & $\mathrm{C}$ & -4.993152 & -1.606414 & 5.333895 \\
\hline $\mathrm{C}$ & -5.668528 & -5.731108 & -2.976846 & $\mathrm{H}$ & -5.911097 & -2.189548 & 5.289757 \\
\hline $\mathrm{H}$ & -6.703613 & -5.980241 & -3.050577 & $\mathrm{C}$ & -3.791832 & -2.213495 & 5.640190 \\
\hline $\mathrm{C}$ & -4.824837 & -5.991254 & -1.866244 & $\mathrm{H}$ & -3.767333 & -3.283406 & 5.853671 \\
\hline $\mathrm{H}$ & -5.109196 & -6.467704 & -0.951691 & $\mathrm{C}$ & -2.585404 & -1.463537 & 5.677757 \\
\hline $\mathrm{C}$ & -3.554091 & -5.485138 & -2.173996 & $\mathrm{C}$ & -1.290272 & -2.076923 & 5.911879 \\
\hline $\mathrm{C}$ & -2.264211 & -5.521104 & -1.405428 & $\mathrm{C}$ & 2.305216 & -1.624446 & 5.777152 \\
\hline $\mathrm{C}$ & -2.381210 & -6.523844 & -0.232993 & $\mathrm{C}$ & 3.525770 & -1.621446 & 6.469734 \\
\hline $\mathrm{C}$ & -3.485709 & -6.811058 & 1.920784 & $\mathrm{H}$ & 3.572724 & -1.912896 & 7.518904 \\
\hline $\mathrm{H}$ & -3.005645 & -7.807168 & 1.850290 & $\mathrm{C}$ & 4.695918 & -1.232445 & 5.809075 \\
\hline $\mathrm{H}$ & -4.580817 & -6.912082 & 2.039124 & $\mathrm{H}$ & 5.637364 & -1.229589 & 6.355417 \\
\hline $\mathrm{C}$ & -2.878446 & -5.959040 & 3.000984 & $\mathrm{C}$ & 4.660033 & -0.841063 & 4.467925 \\
\hline $\mathrm{C}$ & -2.719350 & -6.462316 & 4.294142 & $\mathrm{C}$ & 3.434652 & -0.844914 & 3.777514 \\
\hline
\end{tabular}




\begin{tabular}{|c|c|c|c|c|c|c|c|}
\hline $\mathrm{H}$ & 3.413213 & -0.534391 & 2.723808 & $\mathrm{C}$ & -6.344894 & -5.478802 & -5.933858 \\
\hline $\mathrm{C}$ & 2.266310 & -1.234870 & 4.417283 & $\mathrm{H}$ & -5.928109 & -6.485657 & -6.032699 \\
\hline $\mathrm{H}$ & 1.321479 & -1.244280 & 3.871222 & $\mathrm{H}$ & -6.642913 & -5.127999 & -6.925989 \\
\hline $\mathrm{C}$ & 5.925026 & -0.434669 & 3.762733 & $\mathrm{H}$ & -7.246542 & -5.549552 & -5.315759 \\
\hline $\mathrm{H}$ & 6.541265 & 0.264436 & 4.362506 & $\mathrm{C}$ & -4.098473 & -4.404642 & -6.244373 \\
\hline $\mathrm{H}$ & 6.524544 & -1.311280 & 3.452102 & $\mathrm{H}$ & -3.288175 & -3.787565 & -5.811057 \\
\hline $\mathrm{C}$ & 5.622882 & 1.566334 & 2.401056 & $\mathrm{H}$ & -4.380233 & -3.931847 & -7.188780 \\
\hline $\mathrm{C}$ & 4.994724 & 2.002277 & 1.061622 & $\mathrm{H}$ & -3.660725 & -5.382675 & -6.460312 \\
\hline $\mathrm{C}$ & 6.106392 & 2.410248 & 0.129987 & $\mathrm{C}$ & -1.127894 & -6.012551 & -2.320254 \\
\hline $\mathrm{C}$ & 7.493520 & 2.415011 & 0.318825 & $\mathrm{H}$ & -0.888255 & -5.237961 & -3.079206 \\
\hline $\mathrm{H}$ & 8.015153 & 2.164915 & 1.217073 & $\mathrm{H}$ & -1.408950 & -6.925165 & -2.854932 \\
\hline $\mathrm{C}$ & 8.088613 & 2.836561 & -0.900921 & $\mathrm{H}$ & -0.209238 & -6.216268 & -1.761438 \\
\hline $\mathrm{H}$ & 9.135246 & 2.945190 & -1.076004 & $\mathrm{C}$ & -2.943484 & -0.655821 & 1.715530 \\
\hline $\mathrm{C}$ & 7.056949 & 3.086616 & -1.816655 & $\mathrm{H}$ & -3.687461 & -1.416160 & 1.978047 \\
\hline $\mathrm{C}$ & 7.110209 & 3.551144 & -3.255956 & $\mathrm{H}$ & -3.332913 & 0.319205 & 2.024293 \\
\hline $\mathrm{C}$ & 6.356128 & 4.856906 & -3.360174 & $\mathrm{H}$ & -2.043568 & -0.856034 & 2.306270 \\
\hline $\mathrm{C}$ & 6.796035 & 6.157254 & -3.080645 & $\mathrm{C}$ & -1.584738 & 0.406232 & -0.126387 \\
\hline $\mathrm{H}$ & 7.773858 & 6.439757 & -2.759600 & $\mathrm{H}$ & -0.720201 & 0.352816 & 0.540894 \\
\hline $\mathrm{C}$ & 5.695204 & 7.035041 & -3.270616 & $\mathrm{H}$ & -2.016554 & 1.408237 & -0.042883 \\
\hline $\mathrm{H}$ & 5.705085 & 8.091219 & -3.117625 & $\mathrm{H}$ & -1.203121 & 0.290772 & -1.159627 \\
\hline $\mathrm{C}$ & 4.594571 & 6.262363 & -3.665373 & $\mathrm{~N}$ & -2.721506 & -3.074311 & -0.755948 \\
\hline $\mathrm{C}$ & 3.186672 & 6.709964 & -3.979239 & $\mathrm{~N}$ & -3.889385 & -0.314189 & -1.980996 \\
\hline $\mathrm{C}$ & 2.359565 & 6.758966 & -2.712853 & $\mathrm{~N}$ & -3.614142 & -4.911715 & -3.447552 \\
\hline $\mathrm{C}$ & 1.493076 & 7.761066 & -2.260882 & $\mathrm{~N}$ & -1.133105 & -3.509818 & 5.989710 \\
\hline $\mathrm{H}$ & 1.300262 & 8.697505 & -2.733832 & $\mathrm{~N}$ & 1.069387 & -2.007613 & 6.392652 \\
\hline $\mathrm{C}$ & 0.901915 & 7.311855 & -1.047657 & $\mathrm{~N}$ & 5.843116 & 2.826860 & -1.178763 \\
\hline $\mathrm{H}$ & 0.195294 & 7.858009 & -0.463903 & $\mathrm{~N}$ & 5.007154 & 4.927826 & -3.718215 \\
\hline $\mathrm{C}$ & 1.411158 & 6.038842 & -0.769150 & $\mathrm{~N}$ & 2.310955 & 5.706617 & -1.790953 \\
\hline $\mathrm{C}$ & 1.056121 & 5.090449 & 0.353578 & $\mathrm{~N}$ & 2.957999 & 3.416532 & 0.541034 \\
\hline $\mathrm{C}$ & 2.306106 & 4.554092 & 1.012920 & 0 & -1.744390 & -7.528443 & -0.020871 \\
\hline $\mathrm{C}$ & 3.022531 & 5.024215 & 2.124209 & 0 & -3.371671 & -6.121118 & 0.645761 \\
\hline $\mathrm{H}$ & 2.793587 & 5.889772 & 2.705216 & 0 & 5.576161 & 0.179329 & 2.494965 \\
\hline $\mathrm{C}$ & 4.126683 & 4.154896 & 2.329607 & 0 & 6.115988 & 2.209789 & 3.294402 \\
\hline $\mathrm{H}$ & 4.875371 & 4.243706 & 3.092152 & $\mathrm{H}$ & -2.810415 & -4.435979 & -3.937111 \\
\hline $\mathrm{C}$ & 4.072089 & 3.158711 & 1.346395 & $\mathrm{H}$ & -4.172881 & -2.143021 & -4.286218 \\
\hline $\mathrm{C}$ & 4.224532 & 0.819652 & 0.443372 & $\mathrm{H}$ & -3.095486 & -0.558908 & -2.596205 \\
\hline $\mathrm{H}$ & 4.899797 & -0.020924 & 0.234871 & $\mathrm{H}$ & -3.658827 & -3.030769 & -1.130430 \\
\hline $\mathrm{H}$ & 3.759486 & 1.100991 & -0.517921 & $\mathrm{H}$ & 2.845266 & 4.838636 & -1.848294 \\
\hline $\mathrm{H}$ & 3.435250 & 0.456730 & 1.112309 & $\mathrm{H}$ & 4.413584 & 4.115475 & -3.976481 \\
\hline $\mathrm{C}$ & 6.493657 & 2.468835 & -4.162404 & $\mathrm{H}$ & 4.902020 & 2.926454 & -1.599119 \\
\hline $\mathrm{H}$ & 6.988268 & 1.504720 & -4.020113 & $\mathrm{H}$ & 2.676999 & 2.861153 & -0.280620 \\
\hline $\mathrm{H}$ & 6.563536 & 2.743415 & -5.218344 & C & 1.243820 & 1.881118 & -3.191988 \\
\hline $\mathrm{H}$ & 5.418108 & 2.307612 & -3.956020 & $\mathrm{H}$ & 0.698781 & 2.314720 & -4.052593 \\
\hline $\mathrm{C}$ & 8.575648 & 3.781498 & -3.683684 & $\mathrm{H}$ & 0.691823 & 2.186988 & -2.278334 \\
\hline $\mathrm{H}$ & 9.141518 & 2.846064 & -3.660469 & $\mathrm{C}$ & 1.291195 & 0.355663 & -3.314068 \\
\hline $\mathrm{H}$ & 9.073008 & 4.495018 & -3.017707 & $\mathrm{H}$ & 1.795440 & -0.076871 & -2.428725 \\
\hline $\mathrm{H}$ & 8.619116 & 4.187163 & -4.699001 & $\mathrm{H}$ & 1.914344 & 0.076781 & -4.187800 \\
\hline $\mathrm{C}$ & 2.504682 & 5.757594 & -4.980905 & $\mathrm{C}$ & -0.116328 & -0.237747 & -3.463671 \\
\hline $\mathrm{H}$ & 2.464681 & 4.719222 & -4.611389 & $\mathrm{H}$ & -0.619843 & 0.184795 & -4.355194 \\
\hline $\mathrm{H}$ & 3.043554 & 5.726882 & -5.932051 & $\mathrm{H}$ & -0.750970 & 0.058599 & -2.594362 \\
\hline $\mathrm{H}$ & 1.472996 & 6.062478 & -5.175985 & C & -0.085577 & -1.762236 & -3.566378 \\
\hline $\mathrm{C}$ & 0.209386 & 3.938093 & -0.231623 & $\mathrm{H}$ & 0.203806 & -2.213233 & -2.593177 \\
\hline $\mathrm{H}$ & -0.719425 & 4.320901 & -0.667700 & $\mathrm{H}$ & 0.681285 & -2.098036 & -4.289921 \\
\hline $\mathrm{H}$ & -0.052963 & 3.205549 & 0.537536 & C & 2.644336 & 2.481224 & -3.149915 \\
\hline $\mathrm{H}$ & 0.736805 & 3.401204 & -1.039146 & $\mathrm{C}$ & -1.425167 & -2.364728 & -3.968659 \\
\hline C & 0.217682 & 5.827096 & 1.420442 & 0 & -1.426626 & -3.557098 & -4.375262 \\
\hline $\mathrm{H}$ & 0.018059 & 5.171140 & 2.273886 & 0 & 3.263174 & 2.717315 & -4.209397 \\
\hline $\mathrm{H}$ & -0.742128 & 6.155264 & 1.010544 & 0 & 3.119749 & 2.790318 & -2.012609 \\
\hline $\mathrm{H}$ & 0.746706 & 6.712085 & 1.791322 & 0 & -2.481736 & -1.680366 & -3.876255 \\
\hline
\end{tabular}




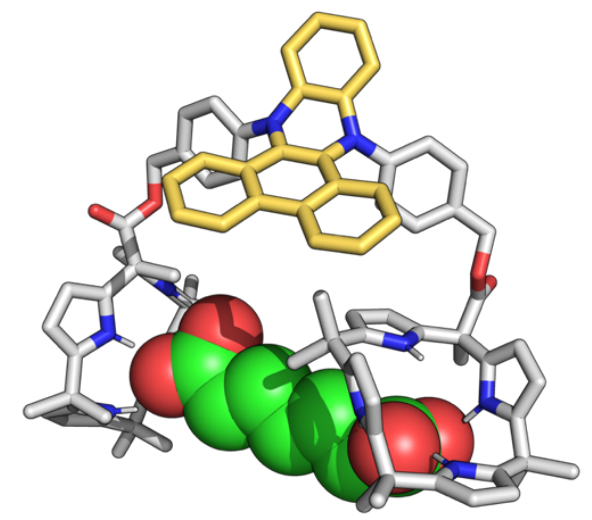

Figure S31. Optimized structure for DPAC-bisC4P $\supset C 7^{2-}$.

Cartesian coordinates of the optimized complex DPAC-bisC4P $\supset \mathbf{C 7}^{2-}$ :

\begin{tabular}{|c|c|c|c|c|c|c|c|}
\hline Symbol & $\mathrm{X}$ & $Y$ & $Z$ & & $\mathrm{X}$ & $Y$ & $Z$ \\
\hline $\mathrm{C}$ & -1.135114 & -4.007015 & -1.979233 & $\mathrm{C}$ & -0.957131 & -7.331351 & 2.790744 \\
\hline $\mathrm{C}$ & 0.012465 & -3.373641 & -1.480336 & $\mathrm{H}$ & -0.978392 & -8.418985 & 2.817264 \\
\hline $\mathrm{H}$ & 1.024549 & -3.691976 & -1.617502 & $\mathrm{C}$ & -0.934152 & -6.622596 & 3.996799 \\
\hline $\mathrm{C}$ & 5.967127 & 7.464641 & -4.705630 & $\mathrm{H}$ & -0.944131 & -7.169940 & 4.938843 \\
\hline $\mathrm{H}$ & 5.243423 & 8.185586 & -5.097835 & $\mathrm{C}$ & -0.906917 & -5.219581 & 3.977971 \\
\hline $\mathrm{H}$ & 6.397822 & 6.915720 & -5.547928 & $\mathrm{C}$ & -0.892145 & -4.535128 & 2.739894 \\
\hline $\mathrm{H}$ & 6.773703 & 8.027892 & -4.222852 & $\mathrm{H}$ & -0.856175 & -3.444345 & 2.711578 \\
\hline $\mathrm{C}$ & -0.409478 & -2.222261 & -0.770147 & $\mathrm{C}$ & -0.925753 & -5.250846 & 1.550747 \\
\hline $\mathrm{H}$ & 0.231051 & -1.519116 & -0.279446 & $\mathrm{H}$ & -0.928970 & -4.718435 & 0.588253 \\
\hline $\mathrm{C}$ & -1.809944 & -2.159986 & -0.843253 & $\mathrm{C}$ & -0.151450 & -4.727439 & 6.353379 \\
\hline $\mathrm{C}$ & -2.703950 & -1.119985 & -0.208302 & $\mathrm{C}$ & -0.010858 & -5.998931 & 6.898527 \\
\hline $\mathrm{C}$ & -4.137801 & -1.202330 & -0.671203 & $\mathrm{H}$ & -0.491098 & -6.859989 & 6.427116 \\
\hline $\mathrm{C}$ & -5.310732 & -1.389887 & 0.069468 & $\mathrm{C}$ & 0.770435 & -6.188614 & 8.053087 \\
\hline $\mathrm{H}$ & -5.374628 & -1.600807 & 1.114627 & $\mathrm{H}$ & 0.886631 & -7.193353 & 8.452272 \\
\hline $\mathrm{C}$ & -6.412536 & -1.255843 & -0.818294 & $\mathrm{C}$ & 1.383690 & -5.109704 & 8.672160 \\
\hline $\mathrm{H}$ & -7.441944 & -1.354312 & -0.554941 & $\mathrm{H}$ & 1.988420 & -5.255541 & 9.563913 \\
\hline $\mathrm{C}$ & -5.903110 & -0.985743 & -2.095235 & $\mathrm{C}$ & 1.232041 & -3.809973 & 8.155251 \\
\hline $\mathrm{N}$ & -5.759885 & -2.818482 & -4.555757 & $\mathrm{H}$ & 1.716915 & -2.971552 & 8.659896 \\
\hline $\mathrm{C}$ & -6.844034 & -2.062440 & -4.101325 & $\mathrm{C}$ & 0.484243 & -3.603414 & 7.001134 \\
\hline $\mathrm{C}$ & -8.017049 & -2.753500 & -4.428864 & $\mathrm{C}$ & -1.030867 & -2.109813 & 5.841050 \\
\hline $\mathrm{H}$ & -9.016874 & -2.447309 & -4.220258 & $\mathrm{C}$ & -1.709037 & -0.828253 & 5.950036 \\
\hline $\mathrm{C}$ & -7.640568 & -3.956928 & -5.088503 & $\mathrm{C}$ & -1.173530 & 0.269850 & 6.675300 \\
\hline $\mathrm{H}$ & -8.311613 & -4.699643 & -5.455748 & $\mathrm{H}$ & -0.251493 & 0.146161 & 7.244760 \\
\hline $\mathrm{C}$ & -6.242620 & -3.985578 & -5.154692 & $\mathrm{C}$ & -1.803737 & 1.498394 & 6.676199 \\
\hline $\mathrm{C}$ & -6.647915 & -0.743385 & -3.390482 & $\mathrm{H}$ & -1.372796 & 2.338713 & 7.218265 \\
\hline $\mathrm{C}$ & -5.865727 & 0.221313 & -4.304260 & $\mathrm{C}$ & -3.007508 & 1.677179 & 5.959297 \\
\hline $\mathrm{H}$ & -5.685778 & 1.180053 & -3.812759 & $\mathrm{H}$ & -3.478282 & 2.658118 & 5.937898 \\
\hline $\mathrm{H}$ & -6.405199 & 0.402147 & -5.237262 & $\mathrm{C}$ & -3.573781 & 0.609292 & 5.293942 \\
\hline $\mathrm{H}$ & -4.879238 & -0.193338 & -4.586641 & $\mathrm{H}$ & -4.510541 & 0.741327 & 4.747839 \\
\hline $\mathrm{C}$ & -8.018685 & -0.109536 & -3.066367 & $\mathrm{C}$ & -2.955902 & -0.671069 & 5.296099 \\
\hline $\mathrm{H}$ & -8.633149 & -0.785582 & -2.462126 & $\mathrm{C}$ & -3.612237 & -1.816051 & 4.677570 \\
\hline $\mathrm{H}$ & -8.569904 & 0.116239 & -3.983555 & $\mathrm{C}$ & -4.891812 & -1.685786 & 4.071803 \\
\hline $\mathrm{H}$ & -7.889883 & 0.819510 & -2.502741 & $\mathrm{H}$ & -5.345838 & -0.694918 & 4.002529 \\
\hline $\mathrm{C}$ & -5.304761 & -5.023108 & -5.726483 & $\mathrm{C}$ & -5.556931 & -2.783523 & 3.565106 \\
\hline $\mathrm{C}$ & -4.403169 & -5.514951 & -4.615027 & $\mathrm{H}$ & -6.528123 & -2.672527 & 3.083858 \\
\hline $\mathrm{C}$ & -4.632473 & -6.515708 & -3.656055 & $\mathrm{C}$ & -4.972575 & -4.066022 & 3.662359 \\
\hline $\mathrm{H}$ & -5.511769 & -7.113626 & -3.564739 & $\mathrm{H}$ & -5.510463 & -4.929612 & 3.273613 \\
\hline $\mathrm{C}$ & -3.493126 & -6.574502 & -2.813454 & $\mathrm{C}$ & -3.722598 & -4.215789 & 4.228743 \\
\hline $\mathrm{H}$ & -3.360185 & -7.219402 & -1.969828 & $\mathrm{H}$ & -3.287001 & -5.213181 & 4.305440 \\
\hline $\mathrm{C}$ & -2.580672 & -5.612389 & -3.269281 & $\mathrm{C}$ & -2.998783 & -3.091462 & 4.709872 \\
\hline $\mathrm{C}$ & -1.197351 & -5.268976 & -2.794934 & $\mathrm{C}$ & -1.646625 & -3.192283 & 5.233057 \\
\hline $\mathrm{C}$ & -0.660872 & -6.408391 & -1.896527 & $\mathrm{C}$ & 1.315697 & -1.337141 & 6.246443 \\
\hline $\mathrm{C}$ & -1.009675 & -7.438589 & 0.283747 & $\mathrm{C}$ & 2.144299 & -0.958471 & 7.314687 \\
\hline $\mathrm{H}$ & -0.021488 & -7.871563 & 0.023256 & $\mathrm{H}$ & 2.027310 & -1.416136 & 8.297199 \\
\hline $\mathrm{H}$ & -1.780754 & -8.229811 & 0.296224 & $\mathrm{C}$ & 3.124825 & 0.018303 & 7.117251 \\
\hline $\mathrm{C}$ & -0.962217 & -6.656773 & 1.566395 & $\mathrm{H}$ & 3.760677 & 0.309157 & 7.951203 \\
\hline
\end{tabular}




\begin{tabular}{|c|c|c|c|}
\hline $\mathrm{C}$ & 3.285278 & 0.622757 & 5.866006 \\
\hline $\mathrm{C}$ & 2.459710 & 0.233287 & 4.797405 \\
\hline $\mathrm{H}$ & 2.604456 & 0.701267 & 3.809992 \\
\hline $\mathrm{C}$ & 1.479609 & -0.734183 & 4.978751 \\
\hline $\mathrm{H}$ & 0.842516 & -1.032661 & 4.144526 \\
\hline $\mathrm{C}$ & 4.349229 & 1.668275 & 5.672442 \\
\hline $\mathrm{H}$ & 4.280087 & 2.484198 & 6.419797 \\
\hline $\mathrm{H}$ & 5.365359 & 1.228224 & 5.672834 \\
\hline $\mathrm{C}$ & 3.758441 & 3.507686 & 4.178821 \\
\hline $\mathrm{C}$ & 3.591838 & 3.767211 & 2.667203 \\
\hline $\mathrm{C}$ & 4.954923 & 3.667584 & 2.036747 \\
\hline $\mathrm{C}$ & 6.176042 & 4.168232 & 2.511317 \\
\hline $\mathrm{H}$ & 6.332588 & 4.686046 & 3.432609 \\
\hline $\mathrm{C}$ & 7.160761 & 3.882878 & 1.531003 \\
\hline $\mathrm{H}$ & 8.193486 & 4.147952 & 1.585013 \\
\hline $\mathrm{C}$ & 6.528472 & 3.216036 & 0.469818 \\
\hline $\mathrm{C}$ & 7.118415 & 2.730900 & -0.834137 \\
\hline $\mathrm{C}$ & 7.135814 & 3.870644 & -1.829022 \\
\hline $\mathrm{C}$ & 8.181577 & 4.344355 & -2.629298 \\
\hline $\mathrm{H}$ & 9.184800 & 3.982186 & -2.646430 \\
\hline $\mathrm{C}$ & 7.672068 & 5.406702 & -3.426850 \\
\hline $\mathrm{H}$ & 8.232964 & 5.971150 & -4.137138 \\
\hline $\mathrm{C}$ & 6.319776 & 5.570616 & -3.107085 \\
\hline $\mathrm{C}$ & 5.291461 & 6.503959 & -3.703943 \\
\hline $\mathrm{C}$ & 4.626557 & 7.321655 & -2.621051 \\
\hline $\mathrm{C}$ & 4.945501 & 8.585403 & -2.109941 \\
\hline $\mathrm{H}$ & 5.765242 & 9.201417 & -2.405768 \\
\hline $\mathrm{C}$ & 3.991109 & 8.901324 & -1.102024 \\
\hline $\mathrm{H}$ & 3.976538 & 9.793646 & -0.516743 \\
\hline $\mathrm{C}$ & 3.097578 & 7.827768 & -1.006074 \\
\hline $\mathrm{C}$ & 1.894506 & 7.627123 & -0.113677 \\
\hline C & 2.288115 & 6.875762 & 1.138370 \\
\hline $\mathrm{C}$ & 2.131999 & 7.229453 & 2.485985 \\
\hline $\mathrm{H}$ & 1.738258 & 8.146532 & 2.862896 \\
\hline $\mathrm{C}$ & 2.597975 & 6.145613 & 3.277465 \\
\hline $\mathrm{H}$ & 2.617732 & 6.104398 & 4.348814 \\
\hline $\mathrm{C}$ & 3.033136 & 5.142410 & 2.406547 \\
\hline $\mathrm{C}$ & 2.599149 & 2.709408 & 2.156191 \\
\hline $\mathrm{H}$ & 2.888167 & 1.693198 & 2.469075 \\
\hline $\mathrm{H}$ & 2.553382 & 2.686772 & 1.049092 \\
\hline $\mathrm{H}$ & 1.582136 & 2.911637 & 2.507412 \\
\hline $\mathrm{C}$ & 6.300225 & 1.565384 & -1.422288 \\
\hline $\mathrm{H}$ & 6.294231 & 0.701864 & -0.749956 \\
\hline $\mathrm{H}$ & 6.702287 & 1.247476 & -2.387929 \\
\hline $\mathrm{H}$ & 5.243660 & 1.835435 & -1.590282 \\
\hline $\mathrm{C}$ & 8.558973 & 2.240032 & -0.570198 \\
\hline $\mathrm{H}$ & 8.566461 & 1.483249 & 0.221069 \\
\hline $\mathrm{H}$ & 9.204080 & 3.065364 & -0.250312 \\
\hline $\mathrm{H}$ & 8.995123 & 1.798422 & -1.470436 \\
\hline $\mathrm{C}$ & 4.245463 & 5.652268 & -4.459632 \\
\hline $\mathrm{H}$ & 3.750838 & 4.932070 & -3.788948 \\
\hline $\mathrm{H}$ & 4.720128 & 5.068235 & -5.253842 \\
\hline $\mathrm{H}$ & 3.470073 & 6.278722 & -4.907057 \\
\hline $\mathrm{C}$ & 0.805581 & 6.812036 & -0.848285 \\
\hline $\mathrm{H}$ & 0.494384 & 7.305339 & -1.772133 \\
\hline $\mathrm{H}$ & -0.076015 & 6.679572 & -0.213462 \\
\hline $\mathrm{H}$ & 1.159376 & 5.802349 & -1.108982 \\
\hline $\mathrm{C}$ & 1.314939 & 9.007812 & 0.259195 \\
\hline $\mathrm{H}$ & 0.403347 & 8.903274 & 0.854871 \\
\hline $\mathrm{H}$ & 1.074906 & 9.582503 & -0.640359 \\
\hline $\mathrm{H}$ & 2.035111 & 9.588786 & 0.846408 \\
\hline $\mathrm{C}$ & -6.106606 & -6.217303 & -6.285782 \\
\hline
\end{tabular}

\begin{tabular}{|c|c|c|c|}
\hline $\mathrm{H}$ & -5.431476 & -7.005721 & -6.632381 \\
\hline $\mathrm{H}$ & -6.735787 & -5.906644 & -7.124409 \\
\hline $\mathrm{H}$ & -6.757029 & -6.648753 & -5.517038 \\
\hline $\mathrm{C}$ & -4.484708 & -4.394779 & -6.870961 \\
\hline $\mathrm{H}$ & -3.833021 & -3.574658 & -6.516138 \\
\hline $\mathrm{H}$ & -5.139027 & -3.969231 & -7.635748 \\
\hline $\mathrm{H}$ & -3.827366 & -5.130047 & -7.342559 \\
\hline $\mathrm{C}$ & -0.244741 & -5.143113 & -3.996938 \\
\hline $\mathrm{H}$ & -0.499008 & -4.244033 & -4.595962 \\
\hline $\mathrm{H}$ & -0.313499 & -6.012690 & -4.657846 \\
\hline $\mathrm{H}$ & 0.798777 & -5.036635 & -3.683321 \\
\hline $\mathrm{C}$ & -2.638427 & -1.320990 & 1.320605 \\
\hline $\mathrm{H}$ & -3.026992 & -2.305633 & 1.607871 \\
\hline $\mathrm{H}$ & -3.224940 & -0.561295 & 1.846834 \\
\hline $\mathrm{H}$ & -1.606627 & -1.253961 & 1.680985 \\
\hline $\mathrm{C}$ & -2.157544 & 0.278716 & -0.571778 \\
\hline $\mathrm{H}$ & -1.205497 & 0.481587 & -0.070516 \\
\hline $\mathrm{H}$ & -2.861872 & 1.066036 & -0.289496 \\
\hline $\mathrm{H}$ & -1.970023 & 0.360248 & -1.658751 \\
\hline $\mathrm{N}$ & -2.248570 & -3.257416 & -1.585669 \\
\hline $\mathrm{N}$ & -4.508888 & -0.974544 & -2.002459 \\
\hline $\mathrm{N}$ & -3.152021 & -4.959225 & -4.365419 \\
\hline $\mathrm{N}$ & -0.907780 & -4.429588 & 5.174549 \\
\hline $\mathrm{N}$ & 0.283616 & -2.323874 & 6.389577 \\
\hline $\mathrm{N}$ & 5.176111 & 3.087659 & 0.786932 \\
\hline $\mathrm{N}$ & 5.996641 & 4.629093 & -2.121615 \\
\hline $\mathrm{N}$ & 3.498390 & 6.863346 & -1.934956 \\
\hline $\mathrm{N}$ & 2.848253 & 5.597035 & 1.093429 \\
\hline 0 & 0.301272 & -7.122687 & -2.057333 \\
\hline 0 & -1.448701 & -6.533954 & -0.768858 \\
\hline 0 & 4.235572 & 2.212354 & 4.333178 \\
\hline 0 & 3.545222 & 4.191782 & 5.150082 \\
\hline $\mathrm{H}$ & -2.701990 & -4.171128 & -4.902906 \\
\hline $\mathrm{H}$ & -4.761397 & -2.552268 & -4.464039 \\
\hline $\mathrm{H}$ & -3.858289 & -0.834066 & -2.788609 \\
\hline $\mathrm{H}$ & -3.207824 & -3.476952 & -1.812048 \\
\hline $\mathrm{H}$ & 3.043618 & 5.953613 & -2.082354 \\
\hline $\mathrm{H}$ & 5.076165 & 4.517998 & -1.685840 \\
\hline $\mathrm{H}$ & 4.444959 & 2.640467 & 0.191968 \\
\hline $\mathrm{H}$ & 3.089602 & 5.072091 & 0.242040 \\
\hline $\mathrm{C}$ & 1.828629 & 2.754729 & -2.847732 \\
\hline $\mathrm{H}$ & 2.461469 & 2.712174 & -3.755992 \\
\hline $\mathrm{H}$ & 1.146257 & 3.611957 & -2.993812 \\
\hline $\mathrm{C}$ & 1.038072 & 1.452894 & -2.689165 \\
\hline $\mathrm{H}$ & 0.319903 & 1.550539 & -1.853110 \\
\hline $\mathrm{H}$ & 1.728190 & 0.637830 & -2.383963 \\
\hline $\mathrm{C}$ & 0.308330 & 1.083474 & -3.987261 \\
\hline $\mathrm{H}$ & 1.049217 & 0.914662 & -4.792301 \\
\hline $\mathrm{H}$ & -0.315007 & 1.934223 & -4.321970 \\
\hline $\mathrm{C}$ & -0.569448 & -0.165363 & -3.827233 \\
\hline $\mathrm{H}$ & -1.352514 & 0.010408 & -3.051980 \\
\hline $\mathrm{H}$ & 0.029909 & -1.016915 & -3.444336 \\
\hline $\mathrm{C}$ & -1.226787 & -0.546009 & -5.156803 \\
\hline $\mathrm{H}$ & -0.454780 & -0.813478 & -5.902858 \\
\hline $\mathrm{H}$ & -1.788983 & 0.311347 & -5.569437 \\
\hline $\mathrm{C}$ & 2.742890 & 3.039045 & -1.663495 \\
\hline $\mathrm{C}$ & -2.186877 & -1.721277 & -4.998543 \\
\hline 0 & -3.271970 & -1.539336 & -4.380360 \\
\hline 0 & 3.107457 & 4.245070 & -1.494393 \\
\hline 0 & 3.141722 & 2.120663 & -0.915445 \\
\hline 0 & -1.885974 & -2.817916 & -5.534680 \\
\hline
\end{tabular}


Cartesian coordinates of the optimized complex DPAC-bisC4P $\supset \mathbf{C 8} \mathbf{8}^{2-}$ :

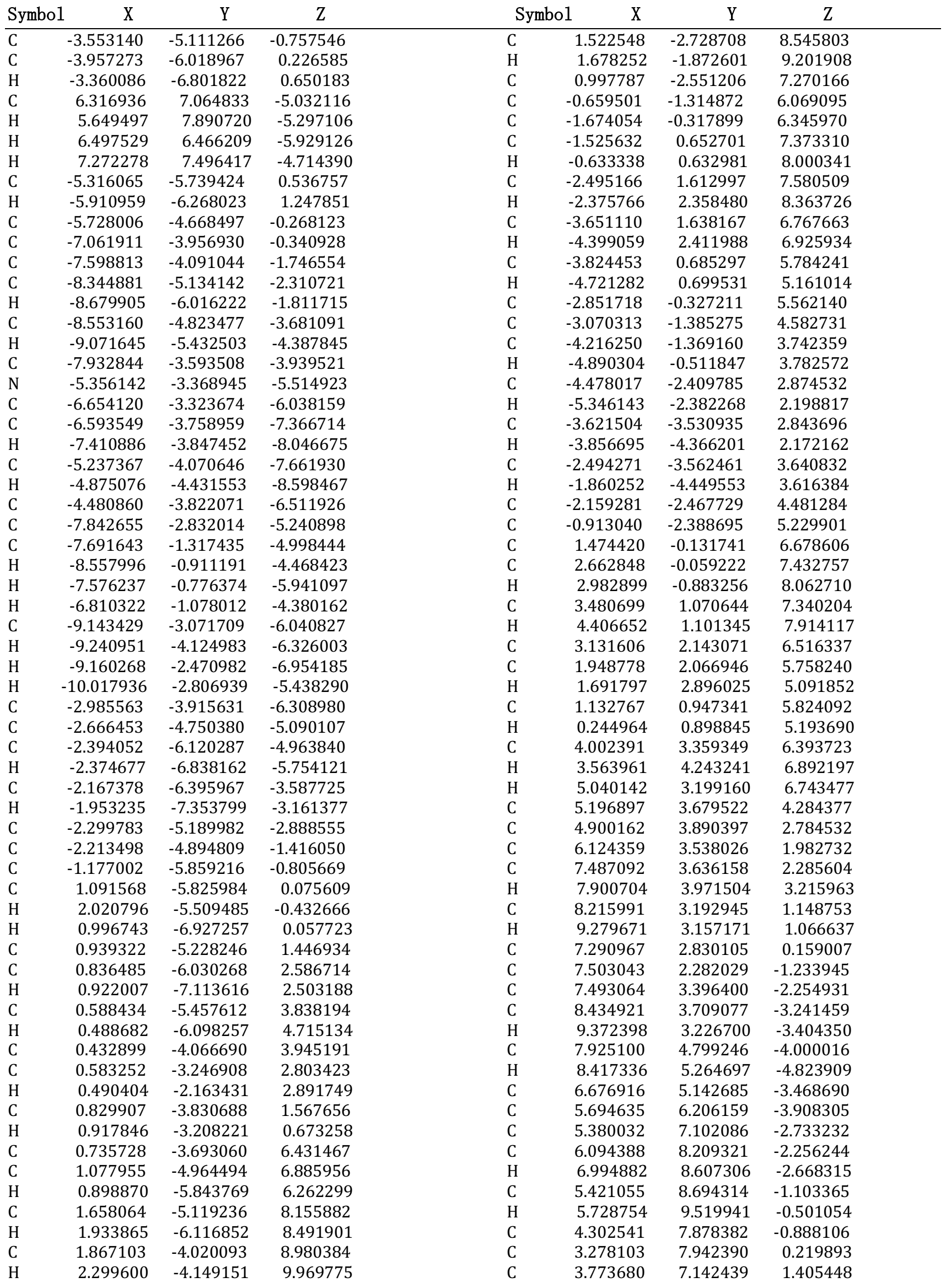




\begin{tabular}{|c|c|c|c|c|c|c|c|}
\hline $\mathrm{C}$ & 3.904614 & 7.512758 & 2.750908 & $\mathrm{H}$ & -7.826216 & -1.950363 & 0.093338 \\
\hline $\mathrm{H}$ & 3.698681 & 8.472125 & 3.169893 & $\mathrm{H}$ & -6.241484 & -1.927248 & -0.667868 \\
\hline $\mathrm{C}$ & 4.364445 & 6.377890 & 3.472147 & $\mathrm{~N}$ & -4.647451 & -4.292859 & -1.068419 \\
\hline $\mathrm{H}$ & 4.553113 & 6.336064 & 4.522902 & $\mathrm{~N}$ & -7.349966 & -3.149806 & -2.749507 \\
\hline $\mathrm{C}$ & 4.508119 & 5.327580 & 2.558869 & $\mathrm{~N}$ & -2.607591 & -4.189742 & -3.814361 \\
\hline $\mathrm{C}$ & 3.719027 & 2.968677 & 2.417704 & $\mathrm{~N}$ & 0.088015 & -3.424108 & 5.180641 \\
\hline $\mathrm{H}$ & 3.965086 & 1.913797 & 2.572746 & $\mathrm{~N}$ & 0.640544 & -1.278827 & 6.705121 \\
\hline $\mathrm{H}$ & 3.426810 & 3.099183 & 1.361790 & $\mathrm{~N}$ & 6.014528 & 3.049985 & 0.674797 \\
\hline $\mathrm{H}$ & 2.835914 & 3.208310 & 3.025332 & $\mathrm{~N}$ & 6.417227 & 4.282667 & -2.395733 \\
\hline $\mathrm{C}$ & 6.378752 & 1.283782 & -1.596249 & $\mathrm{~N}$ & 4.283011 & 6.904005 & -1.890604 \\
\hline $\mathrm{H}$ & 6.323532 & 0.467428 & -0.871241 & $\mathrm{~N}$ & 4.153958 & 5.803289 & 1.290665 \\
\hline $\mathrm{H}$ & 6.546979 & 0.853058 & -2.588154 & 0 & -1.288748 & -6.991590 & -0.403551 \\
\hline $\mathrm{H}$ & 5.395210 & 1.774849 & -1.627539 & 0 & 0.060397 & -5.228904 & -0.760885 \\
\hline $\mathrm{C}$ & 8.856195 & 1.541115 & -1.271766 & 0 & 4.001730 & 3.730742 & 4.984154 \\
\hline $\mathrm{H}$ & 8.897777 & 0.771721 & -0.494083 & 0 & 6.233251 & 3.490320 & 4.873448 \\
\hline $\mathrm{H}$ & 9.686720 & 2.235437 & -1.100231 & $\mathrm{H}$ & -2.776766 & -3.202377 & -3.585339 \\
\hline $\mathrm{H}$ & 9.015302 & 1.059032 & -2.240572 & $\mathrm{H}$ & -5.089549 & -3.117119 & -4.562015 \\
\hline $\mathrm{C}$ & 4.425815 & 5.526267 & -4.457564 & $\mathrm{H}$ & -6.815279 & -2.265023 & -2.626084 \\
\hline $\mathrm{H}$ & 3.893575 & 4.944417 & -3.685430 & $\mathrm{H}$ & -4.646958 & -3.543511 & -1.775984 \\
\hline $\mathrm{H}$ & 4.668197 & 4.832419 & -5.266637 & $\mathrm{H}$ & 3.570907 & 6.153363 & -1.990519 \\
\hline $\mathrm{H}$ & 3.709830 & 6.262401 & -4.834351 & $\mathrm{H}$ & 5.577489 & 4.301427 & -1.808055 \\
\hline $\mathrm{C}$ & 1.921800 & 7.370943 & -0.237158 & $\mathrm{H}$ & 5.131011 & 2.893913 & 0.174071 \\
\hline $\mathrm{H}$ & 1.507532 & 7.946610 & -1.069687 & $\mathrm{H}$ & 4.175140 & 5.252380 & 0.422978 \\
\hline $\mathrm{H}$ & 1.196175 & 7.371432 & 0.580457 & $\mathrm{C}$ & 2.046072 & 2.459532 & -1.383952 \\
\hline $\mathrm{H}$ & 2.003532 & 6.327810 & -0.590996 & $\mathrm{H}$ & 1.938097 & 2.166722 & -0.322379 \\
\hline $\mathrm{C}$ & 3.079537 & 9.418213 & 0.630045 & $\mathrm{H}$ & 2.658913 & 1.665633 & -1.851117 \\
\hline $\mathrm{H}$ & 2.287814 & 9.514953 & 1.378175 & $\mathrm{C}$ & 0.677842 & 2.557320 & -2.060953 \\
\hline $\mathrm{H}$ & 2.807251 & 10.024364 & -0.239676 & $\mathrm{H}$ & 0.813996 & 2.819471 & -3.128969 \\
\hline $\mathrm{H}$ & 3.999302 & 9.835813 & 1.054070 & $\mathrm{H}$ & 0.114848 & 3.408851 & -1.628372 \\
\hline $\mathrm{C}$ & -2.322852 & -4.568113 & -7.540038 & $\mathrm{C}$ & -0.122487 & 1.255469 & -1.919578 \\
\hline $\mathrm{H}$ & -1.248903 & -4.703165 & -7.374820 & $\mathrm{H}$ & -0.250881 & 1.009748 & -0.847924 \\
\hline $\mathrm{H}$ & -2.455991 & -3.951750 & -8.433883 & $\mathrm{H}$ & 0.446513 & 0.413092 & -2.356301 \\
\hline $\mathrm{H}$ & -2.757079 & -5.553731 & -7.742588 & $\mathrm{C}$ & -1.495928 & 1.363850 & -2.596626 \\
\hline $\mathrm{C}$ & -2.429975 & -2.482547 & -6.143905 & $\mathrm{H}$ & -1.366040 & 1.561191 & -3.677701 \\
\hline $\mathrm{H}$ & -2.897046 & -1.957995 & -5.297283 & $\mathrm{H}$ & -2.041347 & 2.240070 & -2.195182 \\
\hline $\mathrm{H}$ & -2.631731 & -1.882085 & -7.036403 & $\mathrm{C}$ & -2.334612 & 0.093703 & -2.393232 \\
\hline $\mathrm{H}$ & -1.349407 & -2.491276 & -5.974987 & $\mathrm{H}$ & -2.451047 & -0.113018 & -1.311143 \\
\hline $\mathrm{C}$ & -1.759425 & -3.447245 & -1.145992 & $\mathrm{H}$ & -1.810223 & -0.786212 & -2.815646 \\
\hline $\mathrm{H}$ & -2.479450 & -2.715557 & -1.554003 & $\mathrm{C}$ & -3.713931 & 0.226599 & -3.041645 \\
\hline $\mathrm{H}$ & -0.781431 & -3.244155 & -1.599737 & $\mathrm{H}$ & -3.617564 & 0.373116 & -4.134040 \\
\hline $\mathrm{H}$ & -1.679293 & -3.252909 & -0.070301 & $\mathrm{H}$ & -4.239062 & 1.124186 & -2.659233 \\
\hline $\mathrm{C}$ & -8.059236 & -4.605363 & 0.643021 & $\mathrm{C}$ & 2.824651 & 3.768863 & -1.448811 \\
\hline $\mathrm{H}$ & -8.168171 & -5.677576 & 0.444309 & $\mathrm{C}$ & -4.598141 & -0.991456 & -2.800507 \\
\hline $\mathrm{H}$ & -9.048592 & -4.147995 & 0.543904 & 0 & -5.789068 & -0.802449 & -2.459820 \\
\hline $\mathrm{H}$ & -7.725921 & -4.487026 & 1.677628 & 0 & 3.935202 & 3.800931 & -0.830022 \\
\hline $\mathrm{C}$ & -6.870535 & -2.480068 & 0.054855 & 0 & 2.392136 & 4.756362 & -2.077278 \\
\hline $\mathrm{H}$ & -6.381343 & -2.391494 & 1.035551 & 0 & -4.133448 & -2.154939 & -2.985826 \\
\hline
\end{tabular}




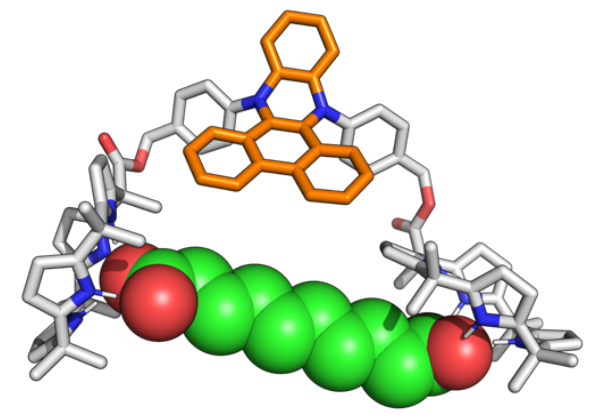

Figure S32. Optimized structure for DPAC-bisC4P $\supset \mathbf{C 1 0}^{2-}$.

Cartesian coordinates of the optimized complex DPAC-bisC4P $\supset \mathbf{C 1 0}{ }^{2-}$ :

\begin{tabular}{|c|c|c|c|c|c|c|c|}
\hline Symbo & $\mathrm{X}$ & $Y$ & Z & & $\mathrm{X}$ & $Y$ & Z \\
\hline $\mathrm{C}$ & -3.202510 & -4.263687 & -1.378592 & $\mathrm{C}$ & -1.017300 & -5.365100 & 4.504048 \\
\hline $\mathrm{C}$ & -2.501890 & -3.283581 & -0.662420 & $\mathrm{H}$ & -1.261249 & -5.467222 & 5.561684 \\
\hline $\mathrm{H}$ & -1.460876 & -3.294246 & -0.419550 & C & -0.225753 & -4.296723 & 4.052859 \\
\hline $\mathrm{C}$ & 4.262590 & 10.141400 & -2.297756 & C & 0.079639 & -4.180563 & 2.677471 \\
\hline $\mathrm{H}$ & 3.356921 & 10.663405 & -1.973983 & $\mathrm{H}$ & 0.693934 & -3.352078 & 2.323332 \\
\hline $\mathrm{H}$ & 4.310382 & 10.179333 & -3.389382 & C & -0.411951 & -5.119131 & 1.778361 \\
\hline $\mathrm{H}$ & 5.123460 & 10.690392 & -1.901369 & $\mathrm{H}$ & -0.165642 & -5.037571 & 0.714968 \\
\hline $\mathrm{C}$ & -3.426012 & -2.266379 & -0.314578 & C & 0.947748 & -3.571231 & 6.174667 \\
\hline $\mathrm{H}$ & -3.195144 & -1.377554 & 0.233291 & C & 0.555314 & -4.564426 & 7.066350 \\
\hline $\mathrm{C}$ & -4.682385 & -2.632599 & -0.820992 & $\mathrm{H}$ & -0.312867 & -5.191214 & 6.850865 \\
\hline $\mathrm{C}$ & -5.958073 & -1.825384 & -0.751575 & C & 1.284098 & -4.776137 & 8.250603 \\
\hline $\mathrm{C}$ & -7.169929 & -2.586529 & -1.230733 & $\mathrm{H}$ & 0.972381 & -5.568104 & 8.927389 \\
\hline $\mathrm{C}$ & -8.242038 & -3.121080 & -0.505731 & $\mathrm{C}$ & 2.381836 & -3.983884 & 8.552781 \\
\hline $\mathrm{H}$ & -8.345159 & -3.141887 & 0.557000 & $\mathrm{H}$ & 2.943052 & -4.144635 & 9.470000 \\
\hline $\mathrm{C}$ & -9.178738 & -3.647426 & -1.436462 & C & 2.782845 & -2.958337 & 7.677454 \\
\hline $\mathrm{H}$ & -10.097804 & -4.131595 & -1.190172 & $\mathrm{H}$ & 3.643656 & -2.337041 & 7.935921 \\
\hline $\mathrm{C}$ & -8.670897 & -3.431324 & -2.724085 & C & 2.093367 & -2.749051 & 6.488064 \\
\hline $\mathrm{N}$ & -7.463950 & -5.457600 & -4.694114 & C & 1.316488 & -1.106160 & 4.878014 \\
\hline $\mathrm{C}$ & -8.812574 & -5.169593 & -4.463519 & C & 1.325188 & 0.317684 & 4.580169 \\
\hline $\mathrm{C}$ & -9.543538 & -6.347468 & -4.660908 & C & 2.366520 & 1.191350 & 4.994802 \\
\hline $\mathrm{H}$ & -10.598727 & -6.467515 & -4.563072 & $\mathrm{H}$ & 3.158941 & 0.823396 & 5.646602 \\
\hline $\mathrm{C}$ & -8.622836 & -7.375333 & -5.008018 & C & 2.390350 & 2.512232 & 4.593212 \\
\hline $\mathrm{H}$ & -8.874584 & -8.391044 & -5.213529 & $\mathrm{H}$ & 3.209150 & 3.176247 & 4.890599 \\
\hline $\mathrm{C}$ & -7.341410 & -6.812238 & -5.017543 & $\mathrm{C}$ & 1.356442 & 3.021755 & 3.777878 \\
\hline $\mathrm{C}$ & -9.271970 & -3.785687 & -4.067500 & $\mathrm{H}$ & 1.404632 & 4.067785 & 3.442554 \\
\hline $\mathrm{C}$ & -8.840616 & -2.774211 & -5.149346 & C & 0.302064 & 2.208610 & 3.417515 \\
\hline $\mathrm{H}$ & -9.133993 & -1.756107 & -4.885010 & $\mathrm{H}$ & -0.506463 & 2.607105 & 2.800745 \\
\hline $\mathrm{H}$ & -9.282902 & -3.025200 & -6.117061 & $\mathrm{C}$ & 0.247194 & 0.849338 & 3.830996 \\
\hline $\mathrm{H}$ & -7.744450 & -2.776397 & -5.296241 & $\mathrm{C}$ & -0.917932 & 0.025001 & 3.534894 \\
\hline $\mathrm{C}$ & -10.811204 & -3.752753 & -3.948637 & C & -2.037747 & 0.568896 & 2.848015 \\
\hline $\mathrm{H}$ & -11.166866 & -4.497138 & -3.227508 & $\mathrm{H}$ & -1.979955 & 1.593925 & 2.474403 \\
\hline $\mathrm{H}$ & -11.281305 & -3.968645 & -4.912343 & C & -3.181700 & -0.175335 & 2.646535 \\
\hline $\mathrm{H}$ & -11.151840 & -2.769789 & -3.609729 & $\mathrm{H}$ & -4.032011 & 0.239942 & 2.103706 \\
\hline $\mathrm{C}$ & -5.992130 & -7.439680 & -5.280349 & C & -3.258132 & -1.495235 & 3.143586 \\
\hline $\mathrm{C}$ & -5.170735 & -7.322088 & -4.014459 & $\mathrm{H}$ & -4.167791 & -2.075640 & 2.987352 \\
\hline $\mathrm{C}$ & -5.190710 & -8.120268 & -2.859172 & $\mathrm{C}$ & -2.178407 & -2.050685 & 3.800278 \\
\hline $\mathrm{H}$ & -5.803284 & -8.979227 & -2.695666 & $\mathrm{H}$ & -2.261959 & -3.065221 & 4.190599 \\
\hline $\mathrm{C}$ & -4.268519 & -7.565403 & -1.935258 & C & -0.972234 & -1.320549 & 3.973037 \\
\hline $\mathrm{H}$ & -4.061439 & -7.925864 & -0.948863 & C & 0.217324 & -1.893821 & 4.581514 \\
\hline $\mathrm{C}$ & -3.691512 & -6.438621 & -2.537480 & C & 3.774353 & -1.421959 & 5.131447 \\
\hline $\mathrm{C}$ & -2.638104 & -5.497529 & -2.027236 & C & 4.810536 & -1.220373 & 6.058403 \\
\hline $\mathrm{C}$ & -1.766818 & -6.248914 & -0.995103 & $\mathrm{H}$ & 4.641079 & -1.376714 & 7.124233 \\
\hline $\mathrm{C}$ & -1.759536 & -7.186451 & 1.244191 & $\mathrm{C}$ & 6.057009 & -0.766764 & 5.615178 \\
\hline $\mathrm{H}$ & -0.948215 & -7.813584 & 0.821082 & $\mathrm{H}$ & 6.841355 & -0.568391 & 6.344854 \\
\hline $\mathrm{H}$ & -2.558428 & -7.822618 & 1.667273 & $\mathrm{C}$ & 6.286085 & -0.531942 & 4.257314 \\
\hline $\mathrm{C}$ & -1.223085 & -6.178780 & 2.221794 & $\mathrm{C}$ & 5.270024 & -0.811603 & 3.324420 \\
\hline $\mathrm{C}$ & -1.515459 & -6.293410 & 3.585088 & $\mathrm{H}$ & 5.463035 & -0.649403 & 2.261145 \\
\hline $\mathrm{H}$ & -2.146517 & -7.105515 & 3.942859 & $\mathrm{C}$ & 4.024987 & -1.252623 & 3.748456 \\
\hline
\end{tabular}




\begin{tabular}{|c|c|c|c|}
\hline $\mathrm{H}$ & 3.233157 & -1.446038 & 3.023999 \\
\hline $\mathrm{C}$ & 7.560223 & 0.095996 & 3.764907 \\
\hline $\mathrm{H}$ & 8.196610 & 0.500260 & 4.573097 \\
\hline $\mathrm{H}$ & 8.145536 & -0.569759 & 3.105283 \\
\hline $\mathrm{C}$ & 6.923470 & 2.419935 & 3.411424 \\
\hline $\mathrm{C}$ & 6.120767 & 3.260181 & 2.398038 \\
\hline $\mathrm{C}$ & 7.089282 & 3.830214 & 1.398597 \\
\hline $\mathrm{C}$ & 8.415663 & 4.251660 & 1.549850 \\
\hline $\mathrm{H}$ & 8.989004 & 4.229808 & 2.452232 \\
\hline $\mathrm{C}$ & 8.857664 & 4.735427 & 0.287718 \\
\hline $\mathrm{H}$ & 9.824664 & 5.137602 & 0.079753 \\
\hline $\mathrm{C}$ & 7.796053 & 4.608174 & -0.619406 \\
\hline $\mathrm{C}$ & 7.702321 & 4.960341 & -2.086690 \\
\hline $\mathrm{C}$ & 7.108862 & 6.338778 & -2.265854 \\
\hline $\mathrm{C}$ & 7.609387 & 7.438429 & -2.971838 \\
\hline $\mathrm{H}$ & 8.548841 & 7.500940 & -3.473430 \\
\hline $\mathrm{C}$ & 6.640803 & 8.477898 & -2.902518 \\
\hline $\mathrm{H}$ & 6.735739 & 9.444027 & -3.344544 \\
\hline $\mathrm{C}$ & 5.558344 & 8.003428 & -2.153449 \\
\hline $\mathrm{C}$ & 4.254504 & 8.681258 & -1.793355 \\
\hline $\mathrm{C}$ & 4.104943 & 8.689492 & -0.289798 \\
\hline $\mathrm{C}$ & 4.638061 & 9.588212 & 0.643905 \\
\hline $\mathrm{H}$ & 5.245039 & 10.439306 & 0.428605 \\
\hline $\mathrm{C}$ & 4.250780 & 9.147800 & 1.937757 \\
\hline $\mathrm{H}$ & 4.516941 & 9.612176 & 2.861381 \\
\hline $\mathrm{C}$ & 3.484794 & 7.985090 & 1.781627 \\
\hline $\mathrm{C}$ & 2.871024 & 7.103508 & 2.843219 \\
\hline $\mathrm{C}$ & 3.890885 & 6.078347 & 3.291609 \\
\hline $\mathrm{C}$ & 4.333427 & 5.748264 & 4.579713 \\
\hline $\mathrm{H}$ & 4.039933 & 6.215331 & 5.493315 \\
\hline $\mathrm{C}$ & 5.262207 & 4.678886 & 4.461195 \\
\hline $\mathrm{H}$ & 5.799362 & 4.214848 & 5.265036 \\
\hline $\mathrm{C}$ & 5.371368 & 4.361560 & 3.104100 \\
\hline $\mathrm{C}$ & 5.088617 & 2.326748 & 1.732153 \\
\hline $\mathrm{H}$ & 5.581619 & 1.498794 & 1.208538 \\
\hline $\mathrm{H}$ & 4.472784 & 2.871167 & 0.996711 \\
\hline $\mathrm{H}$ & 4.400426 & 1.903991 & 2.475003 \\
\hline $\mathrm{C}$ & 6.802841 & 3.943591 & -2.827053 \\
\hline $\mathrm{H}$ & 7.170724 & 2.921870 & -2.702152 \\
\hline $\mathrm{H}$ & 6.764069 & 4.167644 & -3.897655 \\
\hline $\mathrm{H}$ & 5.765946 & 3.976379 & -2.461820 \\
\hline $\mathrm{C}$ & 9.117786 & 4.908235 & -2.698394 \\
\hline $\mathrm{H}$ & 9.579459 & 3.931108 & -2.524371 \\
\hline $\mathrm{H}$ & 9.765988 & 5.669985 & -2.250368 \\
\hline $\mathrm{H}$ & 9.087320 & 5.086117 & -3.777221 \\
\hline $\mathrm{C}$ & 3.090988 & 7.934355 & -2.473394 \\
\hline $\mathrm{H}$ & 2.986505 & 6.897550 & -2.108494 \\
\hline $\mathrm{H}$ & 3.236726 & 7.876756 & -3.555045 \\
\hline $\mathrm{H}$ & 2.133280 & 8.425697 & -2.278070 \\
\hline $\mathrm{C}$ & 1.626218 & 6.364799 & 2.313505 \\
\hline $\mathrm{H}$ & 0.804157 & 7.057874 & 2.116322 \\
\hline $\mathrm{H}$ & 1.280099 & 5.606126 & 3.030164 \\
\hline $\mathrm{H}$ & 1.820819 & 5.836912 & 1.362618 \\
\hline $\mathrm{C}$ & 2.451944 & 7.982030 & 4.041802 \\
\hline $\mathrm{H}$ & 1.925668 & 7.392767 & 4.797986 \\
\hline $\mathrm{H}$ & 1.790469 & 8.790102 & 3.713487 \\
\hline $\mathrm{H}$ & 3.325897 & 8.440291 & 4.518322 \\
\hline $\mathrm{C}$ & -6.154220 & -8.932663 & -5.634072 \\
\hline $\mathrm{H}$ & -5.175894 & -9.406896 & -5.760415 \\
\hline $\mathrm{H}$ & -6.720252 & -9.054943 & -6.561859 \\
\hline $\mathrm{H}$ & -6.684968 & -9.471021 & -4.841201 \\
\hline $\mathrm{C}$ & -5.311435 & -6.719724 & -6.460768 \\
\hline $\mathrm{H}$ & -5.098979 & -5.656524 & -6.239232 \\
\hline $\mathrm{H}$ & -5.948121 & -6.733733 & -7.348968 \\
\hline
\end{tabular}

\begin{tabular}{|c|c|c|c|}
\hline $\mathrm{H}$ & -4.352768 & -7.180854 & -6.712928 \\
\hline $\mathrm{C}$ & -1.693614 & -5.065493 & -3.164511 \\
\hline $\mathrm{H}$ & -2.239786 & -4.484023 & -3.934584 \\
\hline $\mathrm{H}$ & -1.243203 & -5.930746 & -3.663204 \\
\hline $\mathrm{H}$ & -0.882330 & -4.428354 & -2.798053 \\
\hline $\mathrm{C}$ & -6.184448 & -1.393209 & 0.714222 \\
\hline $\mathrm{H}$ & -6.225019 & -2.267502 & 1.373602 \\
\hline $\mathrm{H}$ & -7.124156 & -0.842679 & 0.819220 \\
\hline $\mathrm{H}$ & -5.371729 & -0.748819 & 1.067467 \\
\hline $\mathrm{C}$ & -5.764511 & -0.566752 & -1.629164 \\
\hline $\mathrm{H}$ & -4.966223 & 0.070877 & -1.235716 \\
\hline $\mathrm{H}$ & -6.683007 & 0.023460 & -1.682563 \\
\hline $\mathrm{H}$ & -5.471559 & -0.836984 & -2.652998 \\
\hline $\mathrm{N}$ & -4.539433 & -3.862502 & -1.467881 \\
\hline $\mathrm{N}$ & -7.431544 & -2.800813 & -2.590050 \\
\hline $\mathrm{N}$ & -4.259400 & -6.290399 & -3.807427 \\
\hline $\mathrm{N}$ & 0.285850 & -3.290344 & 4.937574 \\
\hline $\mathrm{N}$ & 2.438028 & -1.751324 & 5.520631 \\
\hline $\mathrm{N}$ & 6.716375 & 4.056888 & 0.070950 \\
\hline $\mathrm{N}$ & 5.850631 & 6.692538 & -1.761311 \\
\hline $\mathrm{N}$ & 3.398846 & 7.710079 & 0.413084 \\
\hline $\mathrm{N}$ & 4.538665 & 5.233867 & 2.388122 \\
\hline 0 & -0.610143 & -6.592084 & -1.101963 \\
\hline 0 & -2.467158 & -6.512758 & 0.162810 \\
\hline 0 & 7.177089 & 1.169545 & 2.857728 \\
\hline 0 & 7.323222 & 2.676248 & 4.520347 \\
\hline $\mathrm{H}$ & -4.037660 & -5.524107 & -4.484728 \\
\hline $\mathrm{H}$ & -6.685156 & -4.775637 & -4.636962 \\
\hline $\mathrm{H}$ & -6.804169 & -2.545975 & -3.367253 \\
\hline $\mathrm{H}$ & -5.275168 & -4.379994 & -1.927824 \\
\hline $\mathrm{H}$ & 2.895443 & 6.907516 & -0.013397 \\
\hline $\mathrm{H}$ & 5.241118 & 6.092632 & -1.194482 \\
\hline $\mathrm{H}$ & 5.787557 & 3.859973 & -0.324006 \\
\hline $\mathrm{H}$ & 4.431671 & 5.245964 & 1.367106 \\
\hline $\mathrm{C}$ & 2.505800 & 3.052752 & -1.134193 \\
\hline $\mathrm{H}$ & 2.389584 & 2.481822 & -0.191154 \\
\hline $\mathrm{H}$ & 3.325684 & 2.557744 & -1.686143 \\
\hline $\mathrm{C}$ & 1.206594 & 3.015245 & -1.942006 \\
\hline $\mathrm{H}$ & 1.376339 & 3.459676 & -2.940757 \\
\hline $\mathrm{H}$ & 0.454690 & 3.670386 & -1.454381 \\
\hline $\mathrm{C}$ & 0.672392 & 1.582749 & -2.072481 \\
\hline $\mathrm{H}$ & 0.434457 & 1.181108 & -1.068336 \\
\hline $\mathrm{H}$ & 1.462086 & 0.922889 & -2.479567 \\
\hline $\mathrm{C}$ & -0.572813 & 1.509706 & -2.967565 \\
\hline $\mathrm{H}$ & -0.326697 & 1.874004 & -3.982579 \\
\hline $\mathrm{H}$ & -1.353895 & 2.192113 & -2.582845 \\
\hline $\mathrm{C}$ & -1.120286 & 0.077378 & -3.041337 \\
\hline $\mathrm{H}$ & -1.434873 & -0.258871 & -2.032979 \\
\hline $\mathrm{H}$ & -0.315707 & -0.618573 & -3.347324 \\
\hline $\mathrm{C}$ & -2.300923 & -0.041200 & -4.015628 \\
\hline $\mathrm{H}$ & -1.984705 & 0.261846 & -5.030933 \\
\hline $\mathrm{H}$ & -3.103764 & 0.661166 & -3.723206 \\
\hline $\mathrm{C}$ & -2.847346 & -1.475037 & -4.042216 \\
\hline $\mathrm{H}$ & -3.188613 & -1.768265 & -3.026093 \\
\hline $\mathrm{H}$ & -2.038920 & -2.190307 & -4.298151 \\
\hline $\mathrm{C}$ & -3.993490 & -1.636396 & -5.044279 \\
\hline $\mathrm{H}$ & -3.643617 & -1.418643 & -6.069793 \\
\hline $\mathrm{H}$ & -4.810561 & -0.925293 & -4.830326 \\
\hline $\mathrm{C}$ & 2.932316 & 4.468574 & -0.765882 \\
\hline $\mathrm{C}$ & -4.534031 & -3.065222 & -5.008598 \\
\hline 0 & -3.780284 & -4.016862 & -5.321992 \\
\hline 0 & 4.156913 & 4.634536 & -0.464625 \\
\hline 0 & 2.110506 & 5.407046 & -0.722221 \\
\hline 0 & -5.741483 & -3.226060 & -4.672608 \\
\hline
\end{tabular}




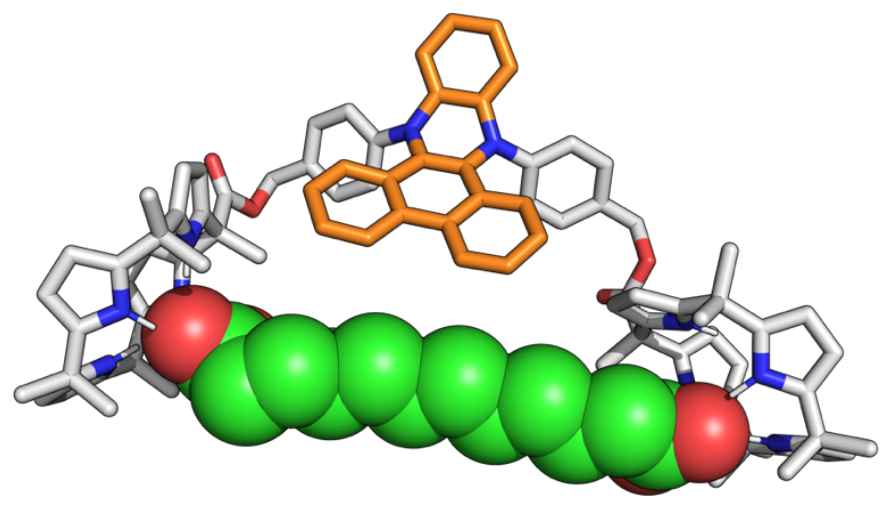

Figure S33. Optimized structure for DPAC-bisC4P $\supset \mathrm{C12}^{2-}$.

Cartesian coordinates of the optimized complex DPAC-bisC4P $\supset \mathbf{C 1 2}^{\mathbf{2}}$ :

\begin{tabular}{|c|c|c|c|c|c|c|c|}
\hline Symbo & $\mathrm{X}$ & $\mathrm{Y}$ & Z & & $\mathrm{X}$ & $Y$ & Z \\
\hline $\mathrm{C}$ & -3.107998 & -5.530205 & -1.412447 & $\mathrm{H}$ & -1.167646 & -8.329381 & 1.583077 \\
\hline $\mathrm{C}$ & -3.089038 & -4.361457 & -0.641389 & $\mathrm{C}$ & -0.314121 & -6.351041 & 1.986113 \\
\hline $\mathrm{H}$ & -2.236424 & -3.747345 & -0.437197 & $\mathrm{C}$ & -0.806608 & -6.324005 & 3.295225 \\
\hline $\mathrm{C}$ & 3.845346 & 12.260730 & -0.578124 & $\mathrm{H}$ & -1.400017 & -7.155566 & 3.673287 \\
\hline $\mathrm{H}$ & 2.907640 & 12.699505 & -0.223511 & $\mathrm{C}$ & -0.560136 & -5.229200 & 4.128358 \\
\hline $\mathrm{H}$ & 3.982521 & 12.541987 & -1.626022 & $\mathrm{H}$ & -0.956889 & -5.221823 & 5.144089 \\
\hline $\mathrm{H}$ & 4.663250 & 12.706756 & -0.000525 & $\mathrm{C}$ & 0.179139 & -4.138106 & 3.643297 \\
\hline $\mathrm{C}$ & -4.408356 & -4.139258 & -0.170216 & $\mathrm{C}$ & 0.674445 & -4.157208 & 2.320030 \\
\hline $\mathrm{H}$ & -4.717225 & -3.326729 & 0.455395 & $\mathrm{H}$ & 1.243456 & -3.308515 & 1.939057 \\
\hline $\mathrm{C}$ & -5.222877 & -5.171758 & -0.658528 & $\mathrm{C}$ & 0.431459 & -5.258588 & 1.507808 \\
\hline $\mathrm{C}$ & -6.689524 & -5.382427 & -0.364529 & $\mathrm{H}$ & 0.823185 & -5.280345 & 0.483944 \\
\hline $\mathrm{C}$ & -7.370367 & -6.348405 & -1.301026 & $\mathrm{C}$ & 0.999958 & -3.059547 & 5.771346 \\
\hline $\mathrm{C}$ & -8.181642 & -7.449968 & -1.000935 & $\mathrm{C}$ & 0.622051 & -4.006875 & 6.717646 \\
\hline $\mathrm{H}$ & -8.372263 & -7.857443 & -0.032706 & $\mathrm{H}$ & -0.143171 & -4.750301 & 6.484254 \\
\hline $\mathrm{C}$ & -8.710737 & -7.945112 & -2.222517 & $\mathrm{C}$ & 1.233344 & -4.021085 & 7.984246 \\
\hline $\mathrm{H}$ & -9.357849 & -8.787701 & -2.326068 & $\mathrm{H}$ & 0.936807 & -4.779668 & 8.704701 \\
\hline $\mathrm{C}$ & -8.219609 & -7.140368 & -3.259540 & $\mathrm{C}$ & 2.197524 & -3.078750 & 8.311139 \\
\hline $\mathrm{N}$ & -6.071305 & -7.711912 & -5.419411 & $\mathrm{H}$ & 2.668208 & -3.087321 & 9.291330 \\
\hline $\mathrm{C}$ & -7.408571 & -8.115748 & -5.360598 & $\mathrm{C}$ & 2.575783 & -2.094926 & 7.380234 \\
\hline $\mathrm{C}$ & -7.495730 & -9.400106 & -5.911995 & $\mathrm{H}$ & 3.327415 & -1.351406 & 7.657889 \\
\hline $\mathrm{H}$ & -8.377402 & -9.990839 & -6.016858 & $\mathrm{C}$ & 2.002989 & -2.078222 & 6.112884 \\
\hline $\mathrm{C}$ & -6.185790 & -9.792752 & -6.302800 & $\mathrm{C}$ & 1.202866 & -0.695682 & 4.292739 \\
\hline $\mathrm{H}$ & -5.924165 & -10.725010 & -6.749847 & $\mathrm{C}$ & 1.053542 & 0.697830 & 3.906093 \\
\hline $\mathrm{C}$ & -5.315210 & -8.743769 & -5.984391 & C & 1.878516 & 1.734494 & 4.420251 \\
\hline $\mathrm{C}$ & -8.480548 & -7.244683 & -4.747824 & $\mathrm{H}$ & 2.607629 & 1.512233 & 5.200621 \\
\hline $\mathrm{C}$ & -8.478274 & -5.855138 & -5.412634 & C & 1.767176 & 3.028263 & 3.951624 \\
\hline $\mathrm{H}$ & -9.223440 & -5.195243 & -4.963906 & $\mathrm{H}$ & 2.415787 & 3.823077 & 4.338668 \\
\hline $\mathrm{H}$ & -8.678967 & -5.933054 & -6.484406 & C & 0.814974 & 3.339154 & 2.955512 \\
\hline $\mathrm{H}$ & -7.496212 & -5.353471 & -5.317003 & $\mathrm{H}$ & 0.766197 & 4.357106 & 2.567061 \\
\hline $\mathrm{C}$ & -9.869230 & -7.885368 & -4.968487 & $\mathrm{C}$ & -0.038835 & 2.359786 & 2.490039 \\
\hline $\mathrm{H}$ & -9.912084 & -8.894801 & -4.545375 & $\mathrm{H}$ & -0.790190 & 2.605310 & 1.736621 \\
\hline $\mathrm{H}$ & -10.095916 & -7.960471 & -6.036039 & C & 0.040966 & 1.025388 & 2.973519 \\
\hline $\mathrm{H}$ & -10.650747 & -7.288254 & -4.489704 & $\mathrm{C}$ & -0.928111 & 0.016420 & 2.563862 \\
\hline $\mathrm{C}$ & -3.816946 & -8.633507 & -6.147347 & $\mathrm{C}$ & -1.967510 & 0.341672 & 1.650869 \\
\hline $\mathrm{C}$ & -3.208537 & -8.455314 & -4.772571 & $\mathrm{H}$ & -1.977458 & 1.334294 & 1.194620 \\
\hline $\mathrm{C}$ & -2.927337 & -9.406918 & -3.778351 & C & -2.952357 & -0.571950 & 1.335148 \\
\hline $\mathrm{H}$ & -3.083315 & -10.460789 & -3.851344 & $\mathrm{H}$ & -3.734234 & -0.323412 & 0.618945 \\
\hline $\mathrm{C}$ & -2.419558 & -8.714975 & -2.648207 & C & -2.948846 & -1.843798 & 1.947317 \\
\hline $\mathrm{H}$ & -2.120222 & -9.152539 & -1.719555 & $\mathrm{H}$ & -3.744942 & -2.556456 & 1.708218 \\
\hline $\mathrm{C}$ & -2.392842 & -7.349345 & -2.966893 & $\mathrm{C}$ & -1.936911 & -2.192170 & 2.819548 \\
\hline $\mathrm{C}$ & -1.960880 & -6.166347 & -2.148007 & $\mathrm{H}$ & -1.961520 & -3.175544 & 3.290322 \\
\hline $\mathrm{C}$ & -0.891243 & -6.655031 & -1.145852 & C & -0.878599 & -1.290936 & 3.109922 \\
\hline $\mathrm{C}$ & -0.578442 & -7.532305 & 1.094749 & C & 0.263983 & -1.648545 & 3.936552 \\
\hline $\mathrm{H}$ & 0.357271 & -7.948452 & 0.671191 & $\mathrm{C}$ & 3.636528 & -0.646947 & 4.786630 \\
\hline
\end{tabular}




\begin{tabular}{|c|c|c|c|}
\hline $\mathrm{C}$ & 4.577145 & -0.310133 & 5.773970 \\
\hline $\mathrm{H}$ & 4.360628 & -0.471650 & 6.830208 \\
\hline $\mathrm{C}$ & 5.785319 & 0.288293 & 5.40251 \\
\hline $\mathrm{H}$ & 6.489530 & 0.591445 & 6.1774 \\
\hline C & 6.073455 & 0.537847 & 4.0583 \\
\hline $\mathrm{C}$ & 5.160868 & 0.130850 & 3.0672 \\
\hline $\mathrm{H}$ & 5.394202 & 0.315640 & 2.0160 \\
\hline $\mathrm{C}$ & 3.957689 & -0.463034 & 3.41903 \\
\hline $\mathrm{H}$ & 3.248329 & -0.760813 & 2.6462 \\
\hline $\mathrm{C}$ & 7.282789 & 1.326378 & 3.6423 \\
\hline $\mathrm{H}$ & 7.886170 & 1.695967 & $4.490 \mathrm{c}$ \\
\hline $\mathrm{H}$ & 7.921471 & 0.796882 & 2.9132 \\
\hline $\mathrm{C}$ & 6.388540 & 3.584196 & 3.5563 \\
\hline $\mathrm{C}$ & 5.546928 & 4.484363 & $2.6273^{\prime}$ \\
\hline $\mathrm{C}$ & 6.503103 & 5.333628 & 1.8362 \\
\hline $\mathrm{C}$ & 7.700440 & 5.931952 & 2.2517 \\
\hline $\mathrm{H}$ & 8.143289 & 5.851965 & 3.2222 \\
\hline $\mathrm{C}$ & 8.200552 & 6.684970 & 1.1585 \\
\hline $\mathrm{H}$ & 9.092296 & 7.272434 & 1.1592 \\
\hline $\mathrm{C}$ & 7.301419 & 6.540428 & 0.09043 \\
\hline $\mathrm{C}$ & 7.350838 & 7.168407 & -1.28305 \\
\hline $\mathrm{C}$ & 6.735978 & 8.549561 & -1.22548 \\
\hline $\mathrm{C}$ & 7.235131 & 9.770643 & -1.69261 \\
\hline $\mathrm{H}$ & 8.181710 & 9.936746 & -2.1559 \\
\hline $\mathrm{C}$ & 6.252866 & 10.767771 & -1.43849 \\
\hline $\mathrm{H}$ & 6.345577 & 11.803020 & -1.6789 \\
\hline $\mathrm{C}$ & 5.162389 & 10.146290 & -0.81980 \\
\hline C & 3.828081 & 10.726454 & -0.41018 \\
\hline C & 3.520849 & 10.401557 & 1.0329 \\
\hline $\mathrm{C}$ & 3.760669 & 11.135000 & 2.2010 \\
\hline $\mathrm{H}$ & 4.246069 & 12.083370 & 2.2672 \\
\hline $\mathrm{C}$ & 3.259971 & 10.381260 & 3.2998 \\
\hline $\mathrm{H}$ & 3.307791 & 10.670579 & 4.3263 \\
\hline $\mathrm{C}$ & 2.718693 & 9.193533 & 2.7939 \\
\hline $\mathrm{C}$ & 2.054658 & 8.036282 & 3.5038 \\
\hline $\mathrm{C}$ & 3.074045 & 6.968899 & 3.83197 \\
\hline $\mathrm{C}$ & 3.402806 & 6.388673 & 5.0644 \\
\hline $\mathrm{H}$ & 3.002081 & 6.647822 & 6.0191 \\
\hline $\mathrm{C}$ & 4.385642 & 5.390458 & 4.8270 \\
\hline $\mathrm{H}$ & 4.864585 & 4.785103 & 5.5712 \\
\hline $\mathrm{C}$ & 4.643323 & 5.367802 & 3.4522 \\
\hline $\mathrm{C}$ & 4.686208 & 3.576292 & 1.7338 \\
\hline $\mathrm{H}$ & 5.308786 & 2.886575 & 1.1480 \\
\hline $\mathrm{H}$ & 4.091066 & 4.154874 & 1.0047 \\
\hline $\mathrm{H}$ & 3.982210 & 2.979614 & 2.3247 \\
\hline $\mathrm{C}$ & 6.582695 & 6.321118 & -2.31613 \\
\hline $\mathrm{H}$ & 7.022109 & 5.324332 & -2.41970 \\
\hline $\mathrm{H}$ & 6.581533 & 6.801813 & -3.2978 \\
\hline $\mathrm{H}$ & 5.527574 & 6.168795 & -2.0324 \\
\hline $\mathrm{C}$ & 8.827256 & 7.267925 & -1.7255 \\
\hline $\mathrm{H}$ & 9.310159 & 6.286585 & -1.6745 \\
\hline $\mathrm{H}$ & 9.387763 & 7.949691 & -1.0764 \\
\hline $\mathrm{H}$ & 8.907161 & 7.636470 & -2.7519 \\
\hline $\mathrm{C}$ & 2.741674 & 10.136457 & -1.34011 \\
\hline $\mathrm{H}$ & 2.721951 & 9.037761 & -1.2877 \\
\hline $\mathrm{H}$ & 2.940153 & 10.399359 & -2.3834 \\
\hline $\mathrm{H}$ & 1.748266 & 10.505034 & -1.0732 \\
\hline $\mathrm{C}$ & 0.959972 & 7.407935 & 2.6102 \\
\hline $\mathrm{H}$ & 0.211043 & 8.149811 & 2.3215 \\
\hline $\mathrm{H}$ & 0.451248 & 6.593679 & 3.1350 \\
\hline $\mathrm{H}$ & 1.385459 & 6.983609 & 1.6872 \\
\hline $\mathrm{C}$ & 1.399182 & 8.557266 & 4.7996 \\
\hline $\mathrm{H}$ & 0.852004 & 7.760995 & 5.3131 \\
\hline $\mathrm{H}$ & 0.699629 & 9.369764 & 4.5809 \\
\hline $\mathrm{H}$ & 2.155363 & 8.944290 & 5.4925 \\
\hline $\mathrm{C}$ & -3.247377 & -9.920299 & 6.77932 \\
\hline
\end{tabular}

\begin{tabular}{|c|c|c|c|}
\hline $\mathrm{H}$ & -2.155432 & -9.870452 & -6.836996 \\
\hline $\mathrm{H}$ & -3.639670 & -10.066362 & -7.789799 \\
\hline $\mathrm{H}$ & -3.509449 & -10.802057 & -6.184418 \\
\hline $\mathrm{C}$ & -3.491791 & -7.438910 & -7.065168 \\
\hline $\mathrm{H}$ & -3.818382 & -6.473295 & -6.631024 \\
\hline $\mathrm{H}$ & -4.000759 & -7.534511 & -8.027854 \\
\hline $\mathrm{H}$ & -2.417670 & -7.352046 & -7.248267 \\
\hline $\mathrm{C}$ & -1.268154 & -5.103972 & -3.020240 \\
\hline $\mathrm{H}$ & -1.990802 & -4.617036 & -3.711200 \\
\hline $\mathrm{H}$ & -0.471558 & -5.546068 & -3.630150 \\
\hline $\mathrm{H}$ & -0.824382 & -4.305822 & -2.416161 \\
\hline $\mathrm{C}$ & -6.790945 & -5.902977 & 1.086832 \\
\hline $\mathrm{H}$ & -6.317185 & -6.887375 & 1.178579 \\
\hline $\mathrm{H}$ & -7.832948 & -5.994167 & 1.404898 \\
\hline $\mathrm{H}$ & -6.278642 & -5.228684 & 1.780766 \\
\hline $\mathrm{C}$ & -7.420319 & -4.023639 & -0.479511 \\
\hline $\mathrm{H}$ & -7.088327 & -3.321950 & 0.289965 \\
\hline $\mathrm{H}$ & -8.502247 & -4.155137 & -0.378869 \\
\hline $\mathrm{H}$ & -7.230175 & -3.559431 & -1.454588 \\
\hline $\mathrm{N}$ & -4.419539 & -6.023352 & -1.418522 \\
\hline $\mathrm{N}$ & -7.384221 & -6.175493 & -2.691304 \\
\hline $\mathrm{N}$ & -2.882830 & -7.201094 & -4.266428 \\
\hline $\mathrm{N}$ & 0.455313 & -2.982354 & 4.447796 \\
\hline $\mathrm{N}$ & 2.326348 & -1.129249 & 5.092207 \\
\hline $\mathrm{N}$ & 6.262878 & 5.709408 & 0.511915 \\
\hline $\mathrm{N}$ & 5.466283 & 8.785456 & -0.685968 \\
\hline $\mathrm{N}$ & 2.889425 & 9.211686 & 1.406676 \\
\hline $\mathrm{N}$ & 3.841859 & 6.342933 & 2.846097 \\
\hline 0 & 0.312976 & -6.640984 & -1.272898 \\
\hline 0 & -1.467931 & -7.140788 & 0.007496 \\
\hline 0 & 6.796927 & 2.455709 & 2.858117 \\
\hline 0 & 6.712854 & 3.723054 & 4.711288 \\
\hline $\mathrm{H}$ & -2.994987 & -6.295067 & -4.770872 \\
\hline $\mathrm{H}$ & -5.707865 & -6.794021 & -5.096867 \\
\hline $\mathrm{H}$ & -6.853592 & -5.459250 & -3.213943 \\
\hline $\mathrm{H}$ & -4.727534 & -6.855801 & -1.900780 \\
\hline $\mathrm{H}$ & 2.607675 & 8.461168 & 0.764205 \\
\hline $\mathrm{H}$ & 4.858982 & 8.079876 & -0.259781 \\
\hline $\mathrm{H}$ & 5.442192 & 5.419323 & -0.056253 \\
\hline $\mathrm{H}$ & 3.826755 & 6.561055 & 1.84012 \\
\hline $\mathrm{C}$ & 1.766103 & 6.098641 & -1.586089 \\
\hline $\mathrm{H}$ & 2.006720 & 5.943896 & -2.654579 \\
\hline $\mathrm{H}$ & 1.358434 & 7.122702 & -1.50199 \\
\hline $\mathrm{C}$ & 0.724451 & 5.079993 & -1.110274 \\
\hline $\mathrm{H}$ & -0.287764 & 5.499563 & -1.267805 \\
\hline $\mathrm{H}$ & 499 & 4.921509 & -0.017888 \\
\hline $\mathrm{C}$ & 0.849171 & 3.736471 & -1.843181 \\
\hline $\mathrm{H}$ & 1.842504 & 3.291386 & -1.624555 \\
\hline $\mathrm{H}$ & 0.838143 & 3.905721 & -2.936463 \\
\hline $\mathrm{C}$ & -0.275355 & 2.769371 & -1.446143 \\
\hline $\mathrm{H}$ & -1.237689 & 3.313434 & -1.392570 \\
\hline $\mathrm{H}$ & -0.086759 & 2.378342 & -0.427447 \\
\hline $\mathrm{C}$ & -0.401996 & 1.602435 & -2.436392 \\
\hline $\mathrm{H}$ & 0.518927 & 0.988784 & -2.412329 \\
\hline $\mathrm{H}$ & -0.474423 & 1.994305 & -3.469191 \\
\hline$c$ & -1.624322 & 0.725509 & -2.127591 \\
\hline $\mathrm{H}$ & -2.529312 & 1.356821 & -2.047735 \\
\hline $\mathrm{H}$ & -1.500771 & 0.243774 & -1.137526 \\
\hline C & -1.837693 & -0.349052 & -3.203357 \\
\hline $\mathrm{H}$ & -0.959531 & -1.022488 & -3.239968 \\
\hline $\mathrm{H}$ & -1.895845 & 0.122771 & -4.202674 \\
\hline $\mathrm{C}$ & -3.108952 & -1.169952 & -2.943399 \\
\hline $\mathrm{H}$ & -3.988106 & -0.501991 & -2.891084 \\
\hline 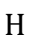 & -3.042759 & -1.664122 & -1.952995 \\
\hline , & 34 & 6.014334 & -0.779217 \\
\hline C & -4.594664 & -4.359696 & -4.568809 \\
\hline
\end{tabular}




$\begin{array}{lrllllll}\mathrm{O} & -3.576306 & -4.709806 & -5.213333 & \mathrm{H} & -2.455422 & -2.929820 & -4.063516 \\ \mathrm{O} & 3.885318 & 5.103100 & -0.988703 & \mathrm{H} & -3.359959 & -1.766088 & -5.031935 \\ \mathrm{O} & 3.265208 & 6.929002 & 0.077839 & \mathrm{C} & -4.597123 & -3.046404 & -3.792898 \\ \mathrm{O} & -5.636502 & -5.073124 & -4.497681 & \mathrm{H} & -4.701164 & -3.300899 & -2.715093 \\ \mathrm{C} & -3.324242 & -2.231448 & -4.029823 & \mathrm{H} & -5.498894 & -2.468257 & -4.054288\end{array}$

Cartesian coordinates of the optimized complex DPAC-bisC4Pدm-Ph ${ }^{2-}$ :

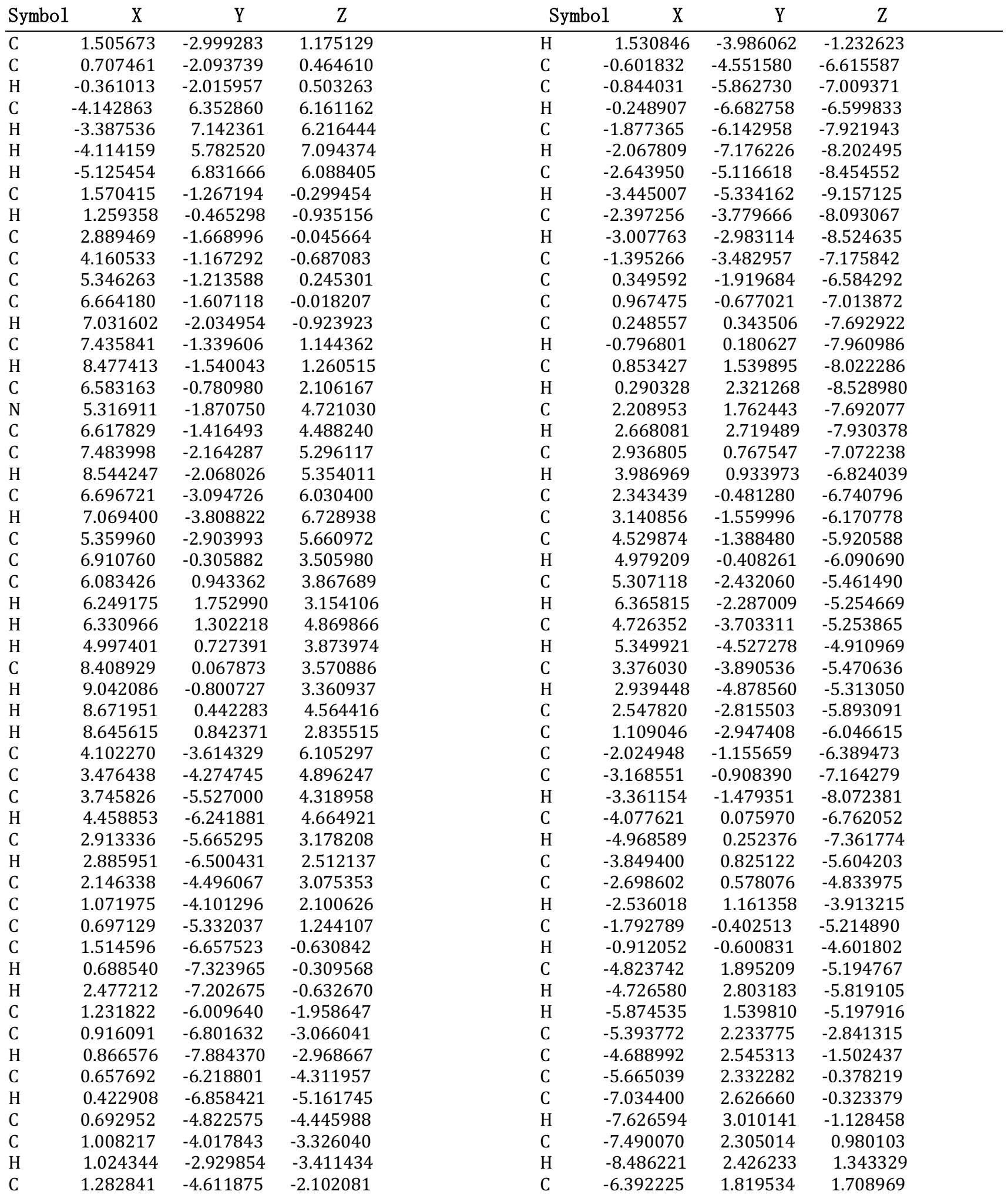




\begin{tabular}{|c|c|c|c|c|c|c|c|}
\hline $\mathrm{C}$ & -6.328877 & 1.367054 & 3.150535 & $\mathrm{H}$ & 3.611867 & -2.066548 & 7.571642 \\
\hline $\mathrm{C}$ & -6.065899 & 2.563639 & 4.037803 & $\mathrm{H}$ & 2.228208 & -3.079176 & 7.110319 \\
\hline $\mathrm{C}$ & -6.763065 & 3.018363 & 5.162900 & $\mathrm{C}$ & -0.190462 & -3.668471 & 2.864887 \\
\hline $\mathrm{H}$ & -7.648136 & 2.592719 & 5.578640 & $\mathrm{H}$ & -0.015375 & -2.695481 & 3.369490 \\
\hline $\mathrm{C}$ & -6.080317 & 4.161581 & 5.665079 & $\mathrm{H}$ & -0.466831 & -4.398362 & 3.632449 \\
\hline $\mathrm{H}$ & -6.372632 & 4.732964 & 6.516860 & $\mathrm{H}$ & -1.051592 & -3.534541 & 2.201308 \\
\hline $\mathrm{C}$ & -4.972457 & 4.392376 & 4.842352 & $\mathrm{C}$ & 4.427561 & -2.049996 & -1.926656 \\
\hline $\mathrm{C}$ & -3.883444 & 5.435208 & 4.947127 & $\mathrm{H}$ & 4.655869 & -3.080236 & -1.632290 \\
\hline $\mathrm{C}$ & -3.862756 & 6.280698 & 3.693705 & $\mathrm{H}$ & 5.266501 & -1.669821 & -2.515371 \\
\hline $\mathrm{C}$ & -4.539441 & 7.467491 & 3.387753 & $\mathrm{H}$ & 3.547725 & -2.082649 & -2.579879 \\
\hline $\mathrm{H}$ & -5.221626 & 7.989755 & 4.020530 & $\mathrm{C}$ & 3.969855 & 0.301799 & -1.125073 \\
\hline $\mathrm{C}$ & -4.174267 & 7.847823 & 2.065742 & $\mathrm{H}$ & 3.231441 & 0.389454 & -1.927259 \\
\hline $\mathrm{H}$ & -4.537596 & 8.704470 & 1.543321 & $\mathrm{H}$ & 4.912175 & 0.730626 & -1.477687 \\
\hline $\mathrm{C}$ & -3.277090 & 6.890900 & 1.575911 & $\mathrm{H}$ & 3.616698 & 0.915164 & -0.285562 \\
\hline $\mathrm{C}$ & -2.565237 & 6.809690 & 0.246266 & $\mathrm{~N}$ & 2.845207 & -2.727374 & 0.865552 \\
\hline $\mathrm{C}$ & -3.299472 & 5.893820 & -0.707800 & $\mathrm{~N}$ & 5.299682 & -0.721904 & 1.554933 \\
\hline $\mathrm{C}$ & -3.705706 & 6.133438 & -2.028402 & $\mathrm{~N}$ & 2.504046 & -3.646542 & 4.123825 \\
\hline $\mathrm{H}$ & -3.604481 & 7.046658 & -2.570507 & $\mathrm{~N}$ & 0.414796 & -4.156949 & -5.684737 \\
\hline $\mathrm{C}$ & -4.285016 & 4.937830 & -2.529243 & $\mathrm{~N}$ & -1.062363 & -2.159032 & -6.739049 \\
\hline $\mathrm{H}$ & -4.675582 & 4.791442 & -3.513287 & $\mathrm{~N}$ & -5.277763 & 1.838607 & 0.873025 \\
\hline $\mathrm{C}$ & -4.227609 & 3.980896 & -1.509061 & $\mathrm{~N}$ & -4.971151 & 3.412541 & 3.841817 \\
\hline $\mathrm{C}$ & -3.491995 & 1.584436 & -1.413136 & $\mathrm{~N}$ & -3.097683 & 5.932108 & 2.577963 \\
\hline $\mathrm{H}$ & -3.811877 & 0.536047 & -1.382736 & $\mathrm{~N}$ & -3.627354 & 4.572141 & -0.392375 \\
\hline $\mathrm{H}$ & -2.901894 & 1.746446 & -0.492816 & 0 & -0.307634 & -6.000229 & 1.249372 \\
\hline $\mathrm{H}$ & -2.808365 & 1.717327 & -2.264653 & 0 & 1.736764 & -5.636701 & 0.376399 \\
\hline $\mathrm{C}$ & -5.210307 & 0.329470 & 3.362404 & 0 & -4.454683 & 2.340817 & -3.864332 \\
\hline $\mathrm{H}$ & -5.378099 & -0.569600 & 2.762494 & 0 & -6.528695 & 1.925579 & -3.105957 \\
\hline $\mathrm{H}$ & -5.136441 & 0.035046 & 4.412801 & $\mathrm{H}$ & 2.097669 & -2.687011 & 4.284306 \\
\hline $\mathrm{H}$ & -4.215948 & 0.714735 & 3.073161 & $\mathrm{H}$ & 4.458146 & -1.498898 & 4.270331 \\
\hline $\mathrm{C}$ & -7.680507 & 0.720098 & 3.524909 & $\mathrm{H}$ & 4.452229 & -0.381891 & 2.036810 \\
\hline $\mathrm{H}$ & -7.921813 & -0.091493 & 2.830808 & $\mathrm{H}$ & 3.639615 & -3.214982 & 1.248373 \\
\hline $\mathrm{H}$ & -8.493824 & 1.452029 & 3.480656 & $\mathrm{H}$ & -2.507588 & 5.093712 & 2.506278 \\
\hline $\mathrm{H}$ & -7.649813 & 0.307635 & 4.537229 & $\mathrm{H}$ & -4.279108 & 3.334787 & 3.088557 \\
\hline $\mathrm{C}$ & -2.530261 & 4.718890 & 5.147185 & $\mathrm{H}$ & -4.314411 & 1.539595 & 1.137256 \\
\hline $\mathrm{H}$ & -2.295779 & 4.038927 & 4.310892 & $\mathrm{H}$ & -3.460441 & 4.104180 & 0.508772 \\
\hline $\mathrm{H}$ & -2.544879 & 4.099861 & 6.049856 & 0 & -2.616930 & 3.344182 & 2.040354 \\
\hline $\mathrm{H}$ & -1.706409 & 5.431055 & 5.232530 & 0 & -2.633028 & 1.178562 & 1.647705 \\
\hline $\mathrm{C}$ & -1.129398 & 6.270398 & 0.433499 & $\mathrm{C}$ & 2.054410 & -0.277672 & 3.634450 \\
\hline $\mathrm{H}$ & -0.550671 & 6.912424 & 1.102671 & 0 & 3.307816 & -0.379110 & 3.488937 \\
\hline $\mathrm{H}$ & -0.607635 & 6.209622 & -0.526730 & 0 & 1.363973 & -1.147473 & 4.216258 \\
\hline $\mathrm{H}$ & -1.121907 & 5.250172 & 0.859904 & $\mathrm{C}$ & -2.028174 & 2.217166 & 1.989827 \\
\hline $\mathrm{C}$ & -2.480398 & 8.229775 & -0.355864 & $\mathrm{H}$ & 2.180246 & 4.191626 & 2.306551 \\
\hline $\mathrm{H}$ & -1.893875 & 8.232175 & -1.278803 & $\mathrm{C}$ & 1.578513 & 3.300169 & 2.449018 \\
\hline $\mathrm{H}$ & -2.011818 & 8.921422 & 0.350897 & $\mathrm{C}$ & 0.024315 & 0.989079 & 2.807774 \\
\hline $\mathrm{H}$ & -3.479681 & 8.614075 & -0.589859 & $\mathrm{C}$ & 2.166952 & 2.124370 & 2.924334 \\
\hline $\mathrm{C}$ & 4.441408 & -4.697595 & 7.150599 & $\mathrm{C}$ & 0.213097 & 3.319715 & 2.155859 \\
\hline $\mathrm{H}$ & 3.543996 & -5.260638 & 7.425643 & $\mathrm{C}$ & -0.564371 & 2.168461 & 2.332731 \\
\hline $\mathrm{H}$ & 4.858921 & -4.248532 & 8.056223 & $\mathrm{C}$ & 1.392071 & 0.972645 & 3.110095 \\
\hline $\mathrm{H}$ & 5.174915 & -5.409299 & 6.756646 & $\mathrm{H}$ & 3.233817 & 2.087742 & 3.151471 \\
\hline $\mathrm{C}$ & 3.136309 & -2.594346 & 6.741002 & $\mathrm{H}$ & -0.265389 & 4.230892 & 1.777639 \\
\hline $\mathrm{H}$ & 2.807235 & -1.822983 & 6.021143 & $\mathrm{H}$ & -0.578437 & 0.084208 & 2.938908 \\
\hline
\end{tabular}

Cartesian coordinates of the optimized complex DPAC-bisC4P $\supset \boldsymbol{p}-\mathbf{P h}^{\mathbf{2}}$ :

\begin{tabular}{lcccccccc} 
Symbo1 & $\mathrm{X}$ & $\mathrm{Y}$ & $\mathrm{Z}$ & \multicolumn{2}{c}{ Symbol } & $\mathrm{X}$ & $\mathrm{Y}$ & $\mathrm{Z}$ \\
\hline $\mathrm{C}$ & -0.908134 & -3.550628 & -1.378132 & $\mathrm{H}$ & 0.619244 & -1.744339 & 0.934695 \\
$\mathrm{C}$ & 0.265320 & -3.220028 & -0.684507 & $\mathrm{C}$ & -1.428543 & -1.924009 & 0.120261 \\
$\mathrm{H}$ & 1.232568 & -3.654027 & -0.833045 & $\mathrm{C}$ & -2.220700 & -0.902886 & 0.905550 \\
$\mathrm{C}$ & 5.813535 & 6.665792 & -6.152530 & $\mathrm{C}$ & -3.696259 & -0.950175 & 0.591685 \\
$\mathrm{H}$ & 5.303203 & 7.613887 & -6.347409 & $\mathrm{C}$ & -4.770509 & -1.420009 & 1.357236 \\
$\mathrm{H}$ & 5.898415 & 6.119385 & -7.095819 & $\mathrm{H}$ & -4.711393 & -1.902281 & 2.309607 \\
$\mathrm{H}$ & 6.825281 & 6.895736 & -5.801464 & $\mathrm{C}$ & -5.967117 & -1.152918 & 0.637526 \\
$\mathrm{C}$ & -0.060424 & -2.207472 & 0.251507 & $\mathrm{H}$ & -6.955094 & -1.402621 & 0.954059
\end{tabular}




\begin{tabular}{|c|c|c|c|}
\hline 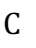 & -5.613684 & -0.523262 & -0.563575 \\
\hline $\mathrm{N}$ & -5.669328 & -1.729980 & -3.372969 \\
\hline $\mathrm{C}$ & -6.727823 & -1.144627 & -2.672507 \\
\hline C & -7.910139 & -1.777666 & -3.074441 \\
\hline $\mathrm{H}$ & -8.896064 & -1.566371 & -2.727811 \\
\hline C & -7.564783 & -2.772513 & -4.032203 \\
\hline $\mathrm{H}$ & -8.249118 & -3.428117 & -4.520841 \\
\hline $\mathrm{C}$ & -6.176152 & -2.734210 & -4.202628 \\
\hline $\mathrm{C}$ & -6.496380 & -0.022849 & -1.686683 \\
\hline $\mathrm{C}$ & -5.837093 & 1.164399 & -2.418338 \\
\hline $\mathrm{H}$ & -5.632739 & 1.993029 & -1.736026 \\
\hline $\mathrm{H}$ & -6.474890 & 1.529588 & -3.227591 \\
\hline $\mathrm{H}$ & -4.875849 & 0.875654 & -2.882530 \\
\hline $\mathrm{C}$ & -7.841318 & 0.446617 & -1.091521 \\
\hline $\mathrm{H}$ & -8.371739 & -0.383824 & -0.613316 \\
\hline $\mathrm{H}$ & -8.490477 & 0.856550 & -1.870557 \\
\hline $\mathrm{H}$ & -7.678591 & 1.220468 & -0.335082 \\
\hline $\mathrm{C}$ & -5.261005 & -3.571887 & -5.065454 \\
\hline $\mathrm{C}$ & -4.327117 & -4.335072 & -4.151269 \\
\hline $\mathrm{C}$ & -4.549785 & -5.533057 & -3.453750 \\
\hline $\mathrm{H}$ & -5.441670 & -6.118973 & -3.472734 \\
\hline $\mathrm{C}$ & -3.383598 & -5.813873 & -2.696793 \\
\hline $\mathrm{H}$ & -3.242169 & -6.649638 & -2.041815 \\
\hline $\mathrm{C}$ & -2.460634 & -4.787560 & -2.939978 \\
\hline $\mathrm{C}$ & -1.052726 & -4.593531 & -2.456060 \\
\hline $\mathrm{C}$ & -0.500396 & -5.896194 & -1.825239 \\
\hline $\mathrm{C}$ & -0.903612 & -7.361276 & 0.088171 \\
\hline $\mathrm{H}$ & 0.084680 & -7.751083 & -0.225258 \\
\hline $\mathrm{H}$ & -1.667041 & 8938 & 0.045923 \\
\hline $\mathrm{C}$ & -0.860387 & -6.684187 & 1.430794 \\
\hline $\mathrm{C}$ & -0.322161 & -7.328843 & 2.545302 \\
\hline $\mathrm{H}$ & 0.078240 & -8.336575 & 2.456262 \\
\hline $\mathrm{C}$ & -0.284600 & -6.691249 & 3.792527 \\
\hline $\mathrm{H}$ & 0.135093 & -7.223350 & 4.643798 \\
\hline $\mathrm{C}$ & -0.768750 & -5.379859 & 3.921059 \\
\hline $\mathrm{C}$ & -1.30 & 538 & 2.789211 \\
\hline $\mathrm{H}$ & -1.66 & 6526 & 2.852517 \\
\hline $\mathrm{C}$ & -1.36 & 838 & 1.569746 \\
\hline $\mathrm{H}$ & -1.792822 & -4.870205 & 0.694386 \\
\hline C & 0.258195 & -4.812779 & 6.179453 \\
\hline $\mathrm{C}$ & 0.704859 & -6.040307 & 6.656036 \\
\hline $\mathrm{H}$ & 0.307837 & -6.970380 & 6.24202 \\
\hline $\mathrm{C}$ & 1.692066 & -6.089586 & 7.65655 \\
\hline $\mathrm{H}$ & 2.047821 & -7.057624 & 8.00106 \\
\hline $\mathrm{C}$ & 2.205836 & -4.918388 & 8.19431 \\
\hline $\mathrm{H}$ & 2.973003 & 5319 & 8.964365 \\
\hline $\mathrm{C}$ & 1.74 & -3.66 & 7.753434 \\
\hline $\mathrm{H}$ & 2.15 & 5631 & 8.196677 \\
\hline $\mathrm{C}$ & 0.780711 & -3.593699 & 6.750104 \\
\hline $\mathrm{C}$ & -1.176403 & -2.417479 & 5.952537 \\
\hline $\mathrm{C}$ & -2.059010 & -1.299155 & 6.244022 \\
\hline $\mathrm{C}$ & -1.622967 & -0.128880 & 6.921102 \\
\hline $\mathrm{H}$ & -0.606866 & -0.079186 & 7.315181 \\
\hline $\mathrm{C}$ & -2.467879 & 0.949539 & 7.09380 \\
\hline $\mathrm{H}$ & -2.119385 & 1.847199 & 7.600625 \\
\hline $\mathrm{C}$ & -3.79 & 758 & 6.600470 \\
\hline $\mathrm{H}$ & -4.436888 & 1.767308 & 6.70812 \\
\hline $\mathrm{C}$ & -4.250655 & -0.249073 & 5.988072 \\
\hline $\mathrm{H}$ & -5.275205 & -0.294494 & 5.611927 \\
\hline $\mathrm{C}$ & -3.409798 & -1.383461 & 5.824997 \\
\hline $\mathrm{C}$ & -3.935729 & -2.630988 & 5.284734 \\
\hline $\mathrm{C}$ & -5.301990 & -2.751860 & 4.911397 \\
\hline $\mathrm{H}$ & -5.939832 & -1.866043 & 4.946376 \\
\hline $\mathrm{C}$ & -5.822896 & -3.960218 & 4.495652 \\
\hline $\mathrm{H}$ & -6.864460 & -4.038633 & 4.189207 \\
\hline $\mathrm{C}$ & -4.998625 & -5.107444 & 4.456937 \\
\hline
\end{tabular}

\begin{tabular}{|c|c|c|c|}
\hline $\mathrm{H}$ & -5.423262 & -6.061072 & 4.148666 \\
\hline $\mathrm{C}$ & -3.662131 & -5.010158 & 4.788083 \\
\hline $\mathrm{H}$ & -3.030687 & -5.899482 & 4.754536 \\
\hline $\mathrm{C}$ & -3.094776 & -3.762984 & 5.164897 \\
\hline $\mathrm{C}$ & -1.674548 & -3.595463 & 5.419390 \\
\hline $\mathrm{C}$ & 1.007901 & -1.197033 & 5.953139 \\
\hline $\mathrm{C}$ & 1.972837 & -0.703982 & 6.846430 \\
\hline $\mathrm{H}$ & 2.173437 & -1.215485 & 7.788240 \\
\hline $\mathrm{C}$ & 2.688364 & 0.452768 & 6.525047 \\
\hline $\mathrm{H}$ & 3.443914 & 0.819874 & 7.217751 \\
\hline $\mathrm{C}$ & 2.437901 & 1.135912 & 5.330346 \\
\hline $\mathrm{C}$ & 1.476197 & 0.635719 & 4.435505 \\
\hline $\mathrm{H}$ & 1.301100 & 1.164777 & 3.492208 \\
\hline $\mathrm{C}$ & 0.768180 & -0.521983 & 4.734915 \\
\hline $\mathrm{H}$ & 0.029059 & -0.909891 & 4.020067 \\
\hline $\mathrm{C}$ & 3.175607 & 2.410096 & 5.032731 \\
\hline $\mathrm{H}$ & 2.687814 & 3.283980 & 5.505643 \\
\hline $\mathrm{H}$ & 4.244530 & 2.365004 & 5.327787 \\
\hline $\mathrm{C}$ & 4.243713 & 2.885230 & 2.895840 \\
\hline $\mathrm{C}$ & 3.898962 & 3.185406 & 1.422844 \\
\hline $\mathrm{C}$ & 5.025034 & 2.690024 & 0.555570 \\
\hline $\mathrm{C}$ & 6.396220 & 2.564400 & 0.809418 \\
\hline $\mathrm{H}$ & 6.886546 & 2.791729 & 1.735877 \\
\hline $\mathrm{C}$ & 7.013063 & 2.081179 & -0.375600 \\
\hline $\mathrm{H}$ & 8.055542 & 1.889653 & -0.502488 \\
\hline $\mathrm{C}$ & 6.013005 & 1.920638 & -1.346183 \\
\hline $\mathrm{C}$ & 6.101093 & 1.446739 & -2.779372 \\
\hline $\mathrm{C}$ & 6.297867 & 2.625179 & -3.705722 \\
\hline $\mathrm{C}$ & 7.287278 & 2.850100 & -4.668601 \\
\hline $\mathrm{H}$ & 8.124232 & 2.223659 & -4.881265 \\
\hline $\mathrm{C}$ & 6.984490 & 4.075612 & -5.325297 \\
\hline $\mathrm{H}$ & 7.557367 & 4.514915 & -6.110318 \\
\hline $\mathrm{C}$ & 5.813392 & 4.588289 & -4.756232 \\
\hline $\mathrm{C}$ & 5.036815 & 5.839663 & -5.102120 \\
\hline $\mathrm{C}$ & 4.863889 & 6.680603 & -3.858879 \\
\hline $\mathrm{C}$ & 5.734614 & 7.631432 & -3.309292 \\
\hline $\mathrm{H}$ & 6.685784 & 7.918768 & -3.698269 \\
\hline $\mathrm{C}$ & 5.138018 & 8.127433 & -2.119193 \\
\hline $\mathrm{H}$ & 5.564194 & 8.851380 & -1.461184 \\
\hline $\mathrm{C}$ & 3.909314 & 7.474258 & -1.954584 \\
\hline $\mathrm{C}$ & 2.901597 & 7.607621 & -0.837152 \\
\hline $\mathrm{C}$ & 3.276339 & 6.659433 & 0.281486 \\
\hline $\mathrm{C}$ & 3.506793 & 6.916263 & 1.640106 \\
\hline $\mathrm{H}$ & 3.476889 & 7.867683 & 2.122019 \\
\hline $\mathrm{C}$ & 3.794871 & 5.677947 & 2.274468 \\
\hline $\mathrm{H}$ & 4.020840 & 5.543422 & 3.309658 \\
\hline $\mathrm{C}$ & 3.734502 & 4.679537 & 1.295065 \\
\hline $\mathrm{C}$ & 2.573267 & 2.491676 & 1.058539 \\
\hline $\mathrm{H}$ & 2.651258 & 1.402727 & 1.135549 \\
\hline $\mathrm{H}$ & 2.264067 & 2.737726 & 0.026719 \\
\hline $\mathrm{H}$ & 1.760409 & 2.827324 & 1.716664 \\
\hline $\mathrm{C}$ & 4.806250 & 0.713161 & -3.192156 \\
\hline $\mathrm{H}$ & 4.597707 & -0.134630 & -2.533644 \\
\hline $\mathrm{H}$ & 4.880062 & 0.335768 & -4.217182 \\
\hline $\mathrm{H}$ & 3.929599 & 1.378250 & -3.170083 \\
\hline $\mathrm{C}$ & 7.288027 & 0.468795 & -2.903638 \\
\hline $\mathrm{H}$ & 7.182650 & -0.357382 & -2.192637 \\
\hline $\mathrm{H}$ & 8.237193 & 0.973607 & -2.692999 \\
\hline $\mathrm{H}$ & 7.346045 & 0.046605 & -3.911260 \\
\hline $\mathrm{C}$ & 3.676626 & 5.438285 & -5.704589 \\
\hline $\mathrm{H}$ & 3.047311 & 4.882427 & -4.987760 \\
\hline $\mathrm{H}$ & 3.806003 & 4.791688 & -6.576692 \\
\hline $\mathrm{H}$ & 3.097740 & 6.316269 & -6.004687 \\
\hline $\mathrm{C}$ & 1.476922 & 7.276416 & -1.321177 \\
\hline $\mathrm{H}$ & 1.149767 & 7.968807 & -2.101466 \\
\hline $\mathrm{H}$ & 0.759316 & 7.314884 & -0.497769 \\
\hline
\end{tabular}




\begin{tabular}{|c|c|c|c|c|c|c|c|}
\hline $\mathrm{H}$ & 1.406322 & 6.263358 & -1.757600 & $\mathrm{~N}$ & 5.397888 & 3.697933 & -3.759064 \\
\hline $\mathrm{C}$ & 2.920754 & 9.063240 & -0.319866 & $\mathrm{~N}$ & 3.747813 & 6.588193 & -3.023470 \\
\hline $\mathrm{H}$ & 2.154283 & 9.218086 & 0.444381 & $\mathrm{~N}$ & 3.422552 & 5.285902 & 0.073596 \\
\hline $\mathrm{H}$ & 2.736078 & 9.762948 & -1.140725 & 0 & 0.549460 & -6.457087 & -2.032552 \\
\hline $\mathrm{H}$ & 3.893071 & 9.312162 & 0.119018 & 0 & -1.384184 & -6.388538 & -0.881803 \\
\hline $\mathrm{C}$ & -6.083395 & -4.578971 & -5.896634 & 0 & 3.067674 & 2.679968 & 3.607797 \\
\hline $\mathrm{H}$ & -5.422027 & -5.232976 & -6.473309 & 0 & 5.304635 & 2.840754 & 3.470097 \\
\hline $\mathrm{H}$ & -6.748576 & -4.059123 & -6.591873 & $\mathrm{H}$ & -2.601790 & -3.000519 & -4.180805 \\
\hline $\mathrm{H}$ & -6.699315 & -5.213665 & -5.250329 & $\mathrm{H}$ & -4.672254 & -1.460542 & -3.291869 \\
\hline $\mathrm{C}$ & -4.478552 & -2.655917 & -6.026745 & $\mathrm{H}$ & -3.666990 & -0.029376 & -1.368657 \\
\hline $\mathrm{H}$ & -3.813696 & -1.954057 & -5.490420 & $\mathrm{H}$ & -2.897451 & -2.755919 & -1.215499 \\
\hline $\mathrm{H}$ & -5.157282 & -2.046875 & -6.629396 & $\mathrm{H}$ & 2.928924 & 5.960136 & -3.170018 \\
\hline $\mathrm{H}$ & -3.839080 & -3.233807 & -6.699875 & $\mathrm{H}$ & 4.574049 & 3.811028 & -3.163695 \\
\hline C & -0.138192 & -4.224404 & -3.637060 & $\mathrm{H}$ & 3.890839 & 2.303605 & -1.243179 \\
\hline $\mathrm{H}$ & -0.369119 & -3.201102 & -3.998515 & $\mathrm{H}$ & 3.321326 & 4.793474 & -0.824821 \\
\hline $\mathrm{H}$ & -0.267821 & -4.909942 & -4.479678 & 0 & 1.576857 & 4.803003 & -3.363146 \\
\hline $\mathrm{H}$ & 0.919758 & -4.231305 & -3.351292 & 0 & 2.839674 & 3.476810 & -2.141721 \\
\hline $\mathrm{C}$ & -2.016407 & -1.181743 & 2.410665 & $\mathrm{C}$ & -2.057856 & -0.580895 & -3.670767 \\
\hline $\mathrm{H}$ & -2.254690 & -2.230204 & 2.654781 & 0 & -3.168306 & -0.492171 & -3.077192 \\
\hline $\mathrm{H}$ & -2.658464 & -0.537670 & 3.021372 & 0 & -1.757744 & -1.525371 & -4.436099 \\
\hline $\mathrm{H}$ & -0.975460 & -1.004231 & 2.718610 & $\mathrm{C}$ & 1.797952 & 3.687981 & -2.833816 \\
\hline $\mathrm{C}$ & -1.666176 & 0.496655 & 0.563079 & $\mathrm{H}$ & -2.122184 & 1.943535 & -4.666525 \\
\hline $\mathrm{H}$ & -0.631063 & 0.611642 & 0.900281 & $\mathrm{C}$ & -1.247447 & 1.773890 & -4.042696 \\
\hline $\mathrm{H}$ & -2.264465 & 1.286300 & 1.026568 & $\mathrm{C}$ & 0.994125 & 1.322122 & -2.425308 \\
\hline $\mathrm{H}$ & -1.656971 & 0.673778 & -0.522435 & $\mathrm{C}$ & -1.051337 & 0.525635 & -3.440396 \\
\hline $\mathrm{N}$ & -1.941450 & -2.745155 & -0.886727 & $\mathrm{C}$ & -0.316821 & 2.794779 & -3.837783 \\
\hline $\mathrm{N}$ & -4.221490 & -0.420198 & -0.593154 & $\mathrm{C}$ & 0.806816 & 2.571175 & -3.030148 \\
\hline $\mathrm{N}$ & -3.052109 & -3.880117 & -3.827210 & $\mathrm{C}$ & 0.065437 & 0.296805 & -2.629114 \\
\hline $\mathrm{N}$ & -0.728823 & -4.651975 & 5.152219 & $\mathrm{H}$ & -0.448355 & 3.776021 & -4.298772 \\
\hline $\mathrm{N}$ & 0.233642 & -2.370118 & 6.240425 & $\mathrm{H}$ & 1.869413 & 1.154263 & -1.797805 \\
\hline $\mathrm{N}$ & 4.801674 & 2.298278 & -0.769869 & $\mathrm{H}$ & 0.208656 & -0.679711 & -2.160308 \\
\hline
\end{tabular}

Cartesian coordinates of the optimized ground-state structure of DPAC-bisC4P:

\begin{tabular}{lccccccc} 
Symbo1 & $\mathrm{X}$ & $\mathrm{Y}$ & $\mathrm{Z}$ & $\mathrm{Symbol}$ & $\mathrm{X}$ & $\mathrm{Y}$ & $\mathrm{Z}$ \\
\hline $\mathrm{C}$ & -6.297000 & 1.490200 & 0.062300 & $\mathrm{C}$ & -3.231100 & -3.995400 & 1.083400 \\
$\mathrm{C}$ & -6.947200 & 1.732200 & 1.277400 & $\mathrm{C}$ & -2.813000 & -4.683200 & 2.395400 \\
$\mathrm{H}$ & -7.971500 & 2.015700 & 1.408400 & $\mathrm{H}$ & -1.718100 & -4.656800 & 2.523700 \\
$\mathrm{C}$ & 6.819100 & 1.062900 & -1.062700 & $\mathrm{H}$ & -3.114200 & -5.736200 & 2.407800 \\
$\mathrm{H}$ & 6.636600 & 2.125900 & -1.264900 & $\mathrm{H}$ & -3.250700 & -4.197500 & 3.272300 \\
$\mathrm{H}$ & 7.903500 & 0.918500 & -1.006900 & $\mathrm{C}$ & -2.555500 & -4.709000 & -0.103300 \\
$\mathrm{H}$ & 6.395200 & 0.859000 & -0.073600 & $\mathrm{H}$ & -2.669000 & -4.156300 & -1.040300 \\
$\mathrm{C}$ & -5.999900 & 1.546700 & 2.320700 & $\mathrm{H}$ & -2.975500 & -5.711200 & -0.246600 \\
$\mathrm{H}$ & -6.193900 & 1.665600 & 3.366400 & $\mathrm{H}$ & -1.477900 & -4.829400 & 0.069000 \\
$\mathrm{C}$ & -4.778500 & 1.194200 & 1.734200 & $\mathrm{C}$ & -7.705300 & -3.477700 & -1.253600 \\
$\mathrm{C}$ & -3.423900 & 0.913600 & 2.345800 & $\mathrm{C}$ & -7.394500 & -2.126100 & -1.854000 \\
$\mathrm{C}$ & -2.861600 & -0.344800 & 1.727500 & $\mathrm{C}$ & -7.285500 & -1.727800 & -3.190200 \\
$\mathrm{C}$ & -1.686100 & -0.559800 & 1.006900 & $\mathrm{H}$ & -7.374700 & -2.355500 & -4.052600 \\
$\mathrm{H}$ & -0.936400 & 0.171900 & 0.779300 & $\mathrm{C}$ & -7.040900 & -0.326700 & -3.212500 \\
$\mathrm{C}$ & -1.640000 & -1.936600 & 0.641500 & $\mathrm{H}$ & -6.911700 & 0.272500 & -4.090300 \\
$\mathrm{H}$ & -0.858000 & -2.412300 & 0.085100 & $\mathrm{C}$ & -6.997800 & 0.117400 & -1.887400 \\
$\mathrm{C}$ & -2.789900 & -2.551000 & 1.140300 & $\mathrm{C}$ & -6.823800 & 1.509500 & -1.344800 \\
$\mathrm{~N}$ & -5.363000 & -3.630300 & -0.264400 & $\mathrm{C}$ & -5.823700 & 2.293000 & -2.226900 \\
$\mathrm{H}$ & -4.900000 & -3.257700 & -1.073700 & $\mathrm{C}$ & -3.546700 & 2.192100 & -3.095500 \\
$\mathrm{C}$ & -4.732900 & -4.048000 & 0.917100 & $\mathrm{H}$ & -3.965100 & 2.888700 & -3.849900 \\
$\mathrm{C}$ & -5.732200 & -4.460100 & 1.802200 & $\mathrm{H}$ & -3.113800 & 1.305900 & -3.596000 \\
$\mathrm{H}$ & -5.590600 & -4.847500 & 2.789900 & $\mathrm{C}$ & -2.603100 & 2.876500 & -2.152000 \\
$\mathrm{C}$ & -6.989200 & -4.296800 & 1.153200 & $\mathrm{C}$ & -3.006900 & 4.029800 & -1.460400 \\
$\mathrm{H}$ & -7.941600 & -4.536100 & 1.578600 & $\mathrm{H}$ & -4.018700 & 4.420900 & -1.607400 \\
$\mathrm{C}$ & -6.750500 & -3.784000 & -0.123800 & $\mathrm{C}$ & -2.128300 & 4.680600 & -0.598200
\end{tabular}




\begin{tabular}{|c|c|c|c|}
\hline $\mathrm{H}$ & -2.449100 & 5.583200 & -0.073700 \\
\hline C & -0.822700 & 4.181000 & -0.416600 \\
\hline C & -0.414800 & 3.024500 & -1.106000 \\
\hline H & 0.595900 & 2.619100 & -0.980400 \\
\hline $\mathrm{C}$ & -1.305300 & 2.383300 & -1.966700 \\
\hline $\mathrm{H}$ & -0.975900 & 1.480300 & -2.497300 \\
\hline $\mathrm{C}$ & -0.265900 & 5.100300 & 1.816100 \\
\hline $\mathrm{C}$ & -1.332900 & 5.930100 & 2.179000 \\
\hline $\mathrm{H}$ & -1.964100 & 6.377600 & 1.407300 \\
\hline C & -1.577600 & 6.190600 & 3.531200 \\
\hline $\mathrm{H}$ & -2.414600 & 6.830400 & 3.810700 \\
\hline $\mathrm{C}$ & -0.748100 & 5.655300 & 4.519000 \\
\hline $\mathrm{H}$ & -0.930200 & 5.886600 & 5.567800 \\
\hline $\mathrm{C}$ & 0.325100 & 4.828000 & 4.170200 \\
\hline $\mathrm{H}$ & 0.988800 & 4.428000 & 4.938200 \\
\hline $\mathrm{C}$ & 0.548200 & 4.518700 & 2.825100 \\
\hline $\mathrm{C}$ & 2.281900 & 4.135400 & 1.211900 \\
\hline $\mathrm{C}$ & 3.728600 & 4.070600 & 1.104800 \\
\hline $\mathrm{C}$ & 4.531800 & 3.540800 & 2.150200 \\
\hline $\mathrm{H}$ & 4.046700 & 3.171600 & 3.057400 \\
\hline $\mathrm{C}$ & 5.906600 & 3.501000 & 2.028800 \\
\hline $\mathrm{H}$ & 6.521300 & 3.099200 & 2.832800 \\
\hline $\mathrm{C}$ & 6.527600 & 3.991100 & 0.857700 \\
\hline $\mathrm{H}$ & 7.613200 & 3.960100 & 0.776200 \\
\hline $\mathrm{C}$ & 5.761600 & 4.509800 & -0.167700 \\
\hline $\mathrm{H}$ & 6.243900 & 4.894800 & -1.068300 \\
\hline $\mathrm{C}$ & 4.344900 & 4.563900 & -0.069400 \\
\hline $\mathrm{C}$ & 3.531300 & 5.131100 & -1.139200 \\
\hline $\mathrm{C}$ & 4.127400 & 5.611100 & -2.336500 \\
\hline $\mathrm{H}$ & 5.208500 & 5.524800 & -2.460700 \\
\hline $\mathrm{C}$ & 3.363000 & 6.182300 & -3.334600 \\
\hline $\mathrm{H}$ & 3.828600 & 6.544800 & -4.250000 \\
\hline $\mathrm{C}$ & 1.965300 & 6.305700 & -3.171000 \\
\hline $\mathrm{H}$ & 1.373000 & 6.770800 & -3.957800 \\
\hline $\mathrm{C}$ & 1.359900 & 5.841000 & -2.020000 \\
\hline $\mathrm{H}$ & 0.280800 & 5.950500 & -1.892600 \\
\hline $\mathrm{C}$ & 2.126400 & 5.230300 & -0.991600 \\
\hline $\mathrm{C}$ & 1.515100 & 4.709700 & 0.222200 \\
\hline $\mathrm{C}$ & 1.442500 & 2.237300 & 2.554100 \\
\hline $\mathrm{C}$ & 0.764200 & 1.727400 & 3.680900 \\
\hline $\mathrm{H}$ & 0.374100 & 2.393600 & 4.448100 \\
\hline $\mathrm{C}$ & 0.583900 & 0.354400 & 3.824400 \\
\hline $\mathrm{H}$ & 0.051500 & -0.025400 & 4.696500 \\
\hline $\mathrm{C}$ & 1.070700 & -0.536600 & 2.854400 \\
\hline $\mathrm{C}$ & 1.770400 & -0.032600 & 1.751600 \\
\hline $\mathrm{H}$ & 2.170200 & -0.721400 & 1.001400 \\
\hline $\mathrm{C}$ & 1.958600 & 1.341200 & 1.598000 \\
\hline $\mathrm{H}$ & 2.512800 & 1.702100 & 0.728700 \\
\hline $\mathrm{C}$ & 0.769600 & -1.993400 & 3.026600 \\
\hline $\mathrm{H}$ & 1.032700 & -2.373800 & 4.030600 \\
\hline $\mathrm{H}$ & -0.295600 & -2.223100 & 2.799800 \\
\hline $\mathrm{C}$ & 1.368100 & -4.088100 & 2.000900 \\
\hline $\mathrm{C}$ & 2.317500 & -4.758000 & 0.982400 \\
\hline $\mathrm{C}$ & 3.744900 & -4.463000 & 1.354200 \\
\hline $\mathrm{C}$ & 4.323700 & -4.261800 & 2.610500 \\
\hline $\mathrm{H}$ & 3.812600 & -4.231900 & 3.551300 \\
\hline $\mathrm{C}$ & 5.723200 & -4.091900 & 2.419200 \\
\hline $\mathrm{H}$ & 6.438800 & -3.921400 & 3.196800 \\
\hline $\mathrm{C}$ & 5.987000 & -4.194600 & 1.050000 \\
\hline $\mathrm{C}$ & 7.290600 & -4.135100 & 0.288500 \\
\hline $\mathrm{C}$ & 7.151200 & -3.199200 & -0.889200 \\
\hline $\mathrm{C}$ & 7.496200 & -3.365200 & -2.232200 \\
\hline $\mathrm{H}$ & 7.918400 & -4.240500 & -2.680900 \\
\hline $\mathrm{C}$ & 7.198100 & -2.151300 & -2.913800 \\
\hline $\mathrm{H}$ & 7.364400 & -1.970000 & -3.955500 \\
\hline $\mathrm{C}$ & 6.675300 & -1.250000 & -1.982500 \\
\hline
\end{tabular}

\begin{tabular}{|c|c|c|c|}
\hline $\mathrm{C}$ & 6.197600 & 0.173200 & -2.156300 \\
\hline$u$ & 4.690600 & 0.227000 & -2.065400 \\
\hline C & 3.852000 & 1.266400 & -1.656500 \\
\hline $\mathrm{H}$ & 4.158200 & 2.227700 & -1.293900 \\
\hline C & 2.505700 & 0.830600 & -1.815700 \\
\hline $\mathrm{H}$ & 1.634200 & 1.418000 & -1.591600 \\
\hline $\mathrm{C}$ & 2.531800 & -0.471200 & -2.320400 \\
\hline C & 1.410300 & -1.403200 & -2.710700 \\
\hline C & 1.624900 & -2.756100 & -2.069000 \\
\hline $\mathrm{C}$ & 1.583900 & -4.038700 & -2.627700 \\
\hline $\mathrm{H}$ & 1.404600 & -4.281800 & -3.654800 \\
\hline C & 1.815300 & -4.977000 & -1.586300 \\
\hline $\mathrm{H}$ & 1.835900 & -6.041700 & -1.700700 \\
\hline $\mathrm{C}$ & 1.997000 & 0500 & -0.398400 \\
\hline $\mathrm{C}$ & 2.080200 & -6.275000 & 1.085900 \\
\hline $\mathrm{H}$ & 2.258600 & -6.632800 & 2.110000 \\
\hline $\mathrm{H}$ & 2.754300 & -6.829600 & 0.423700 \\
\hline $\mathrm{H}$ & 1.046300 & -6.539000 & 0.827200 \\
\hline C & 7.639800 & -5.555300 & -0.196300 \\
\hline $\mathrm{H}$ & 7.659800 & -6.260000 & 0.643900 \\
\hline $\mathrm{H}$ & 8.625600 & -5.582300 & -0.674200 \\
\hline $\mathrm{H}$ & 6.910100 & -5.930500 & -0.921200 \\
\hline C & 8.417300 & -3.612500 & 1.200300 \\
\hline $\mathrm{H}$ & 8.624800 & -4.311200 & 2.018600 \\
\hline $\mathrm{H}$ & 8.165500 & -2.645200 & 1.647200 \\
\hline$H$ & 9.347000 & -3.480400 & 0.633600 \\
\hline C & 6.614300 & 0.700600 & -3.543600 \\
\hline $\mathrm{H}$ & 6.173500 & 0.105500 & -4.351000 \\
\hline $\mathrm{H}$ & 7.702600 & 0.680800 & -3.666100 \\
\hline $\mathrm{H}$ & 6.276900 & 1.734000 & -3.686000 \\
\hline C & 1.393200 & -1.537400 & -4.245700 \\
\hline $\mathrm{H}$ & 2.298600 & -2.023300 & -4.624000 \\
\hline $\mathrm{H}$ & 1.330800 & -0.550600 & -4.720500 \\
\hline $\mathrm{H}$ & 0.533800 & -2.124900 & -4.586700 \\
\hline C & 0.058500 & -0.850500 & -2.228200 \\
\hline $\mathrm{H}$ & -0.752700 & -1.562900 & -2.422100 \\
\hline $\mathrm{H}$ & -0.199600 & 0.092500 & -2.733800 \\
\hline $\mathrm{H}$ & 0.060500 & -0.650200 & -1.148900 \\
\hline C & -7.560800 & -4.578700 & -2.322400 \\
\hline $\mathrm{H}$ & -8.306300 & -4.465000 & -3.117000 \\
\hline $\mathrm{H}$ & -7.698200 & -5.572200 & -1.878300 \\
\hline $\mathrm{H}$ & -6.571000 & -4.563900 & -2.790300 \\
\hline C & -9.155500 & -3.453400 & -0.732400 \\
\hline $\mathrm{H}$ & -9.276800 & 3000 & 0.100500 \\
\hline $\mathrm{H}$ & -9.469900 & -4.442800 & -0.382000 \\
\hline $\mathrm{H}$ & -9.849600 & -3.146400 & -1.524100 \\
\hline C & -8.168700 & 2.250100 & -1.420000 \\
\hline $\mathrm{H}$ & -8.940900 & 1.727500 & -0.843700 \\
\hline $\mathrm{H}$ & -8.524900 & 2.321500 & -2.456900 \\
\hline $\mathrm{H}$ & -8.085300 & 3.274600 & -1.033100 \\
\hline C & -2.502800 & 2.112000 & 5100 \\
\hline $\mathrm{H}$ & -2.360700 & 2.269100 & 0.978600 \\
\hline $\mathrm{H}$ & -1.509700 & 1.968000 & 2.499000 \\
\hline 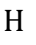 & -2.918800 & 3.039400 & 2.465300 \\
\hline 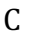 & -3.551500 & 0.720500 & 3.868400 \\
\hline $\mathrm{H}$ & -3.864900 & 1.648100 & 4.360900 \\
\hline $\mathrm{H}$ & -2.588500 & 0.425600 & 4.301800 \\
\hline $\mathrm{H}$ & -4.283100 & -0.051500 & 4.126300 \\
\hline N & -4.965400 & 1.155300 & 0.351100 \\
\hline H & -4.257500 & 0.937300 & -0.342600 \\
\hline $\mathrm{N}$ & -3.536600 & -1.573600 & 1.812000 \\
\hline 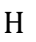 & -4.430000 & -1.722500 & 2.247400 \\
\hline $\mathrm{N}$ & -7.219600 & -0.987700 & -1.056000 \\
\hline $\mathrm{H}$ & -7.225800 & -0.967600 & -0.049800 \\
\hline $\mathrm{N}$ & 0.092800 & 4.922400 & 0.422400 \\
\hline $\mathrm{N}$ & 1.667000 & 3.663600 & 2.447800 \\
\hline
\end{tabular}




\begin{tabular}{rrrrrrrr}
$\mathrm{N}$ & 4.768200 & -4.426400 & 0.398100 & $\mathrm{~N}$ & 1.881900 & -2.895000 & -0.701400 \\
$\mathrm{H}$ & 4.641000 & -4.522500 & -0.595000 & $\mathrm{H}$ & 1.958000 & -2.140300 & -0.022700 \\
$\mathrm{~N}$ & 6.643300 & -1.900000 & -0.740400 & $\mathrm{O}$ & -5.969600 & 3.361200 & -2.766300 \\
$\mathrm{H}$ & 6.294200 & -1.508500 & 0.116200 & $\mathrm{O}$ & -4.638800 & 1.593300 & -2.328400 \\
$\mathrm{~N}$ & 3.877400 & -0.837200 & -2.478100 & 0 & 1.596500 & -2.732600 & 2.061000 \\
$\mathrm{H}$ & 4.206600 & -1.727100 & -2.810600 & 0 & 0.520400 & -4.612300 & 2.681800 \\
\hline
\end{tabular}

Cartesian coordinates of the optimized first excited-state structure of DPAC-bisC4P:

\begin{tabular}{|c|c|c|c|c|c|c|c|}
\hline Symbo] & $X$ & $Y$ & Z & & $\mathrm{X}$ & $Y$ & Z \\
\hline $\bar{C}$ & -6.236400 & 1.218000 & 1.217400 & $\mathrm{H}$ & -0.857200 & 2.818400 & -1.488400 \\
\hline $\mathrm{C}$ & -5.991400 & 1.139400 & 2.592100 & $\mathrm{C}$ & -2.915300 & 3.452500 & -1.699300 \\
\hline $\mathrm{H}$ & -6.599500 & 1.547900 & 3.373800 & $\mathrm{H}$ & -3.034400 & 2.989300 & -2.679300 \\
\hline $\mathrm{C}$ & 1.305800 & -0.060000 & -2.109200 & C & -0.308900 & 3.004800 & 2.171700 \\
\hline $\mathrm{H}$ & 0.338300 & 0.201400 & -1.664900 & C & -1.502700 & 2.670600 & 2.876500 \\
\hline $\mathrm{H}$ & 1.278500 & 0.255900 & -3.157700 & $\mathrm{H}$ & -2.475200 & 2.809100 & 2.400500 \\
\hline $\mathrm{H}$ & 2.057500 & 0.546700 & -1.588000 & $\mathrm{C}$ & -1.442600 & 2.169500 & 4.154500 \\
\hline $\mathrm{C}$ & -4.778000 & 0.423900 & 2.775000 & $\mathrm{H}$ & -2.355100 & 1.926400 & 4.695000 \\
\hline $\mathrm{H}$ & -4.321800 & 0.205000 & 3.718700 & $\mathrm{C}$ & -0.186700 & 1.975700 & 4.795000 \\
\hline $\mathrm{C}$ & -4.286600 & 0.072500 & 1.511200 & $\mathrm{H}$ & -0.170100 & 1.645800 & 5.831700 \\
\hline $\mathrm{C}$ & -3.040900 & -0.697900 & 1.130300 & $\mathrm{C}$ & 0.982800 & 2.199000 & 4.112600 \\
\hline $\mathrm{C}$ & -3.429300 & -1.966900 & 0.408500 & $\mathrm{H}$ & 1.943000 & 2.052900 & 4.606200 \\
\hline $\mathrm{C}$ & -2.841800 & -2.602900 & -0.686700 & C & 0.955900 & 2.631200 & 2.750000 \\
\hline $\mathrm{H}$ & -1.985800 & -2.261700 & -1.244100 & C & 2.054800 & 3.224200 & 0.676600 \\
\hline $\mathrm{C}$ & -3.555100 & -3.811700 & -0.927000 & $\mathrm{C}$ & 3.075400 & 3.053200 & -0.334400 \\
\hline $\mathrm{H}$ & -3.332000 & -4.519000 & -1.698300 & $\mathrm{C}$ & 3.950400 & 1.939100 & -0.373800 \\
\hline $\mathrm{C}$ & -4.571300 & -3.908100 & 0.026400 & $\mathrm{H}$ & 3.829500 & 1.125800 & 0.341100 \\
\hline $\mathrm{N}$ & -7.382600 & -3.523300 & -0.793500 & C & 4.955700 & 1.849900 & -1.322200 \\
\hline $\mathrm{H}$ & -6.788700 & -3.185000 & -1.529300 & $\mathrm{H}$ & 5.659900 & 1.014800 & -1.303200 \\
\hline C & -6.985700 & -4.392200 & 0.232700 & $\mathrm{C}$ & 5.090300 & 2.846400 & -2.305500 \\
\hline $\mathrm{C}$ & -8.085900 & -4.585500 & 1.071000 & $\mathrm{H}$ & 5.897300 & 2.781300 & -3.032800 \\
\hline $\mathrm{H}$ & -8.122200 & -5.199200 & 1.947000 & $\mathrm{C}$ & 4.194100 & 3.902800 & -2.337100 \\
\hline $\mathrm{C}$ & -9.171800 & -3.825500 & 0.550300 & $\mathrm{H}$ & 4.280100 & 4.660000 & -3.119200 \\
\hline $\mathrm{H}$ & -10.154100 & -3.781200 & 0.972900 & $\mathrm{C}$ & 3.168200 & 4.025800 & -1.372100 \\
\hline $\mathrm{C}$ & -8.728400 & -3.174400 & -0.603400 & $\mathrm{C}$ & 2.213600 & 5.127600 & -1.429700 \\
\hline $\mathrm{C}$ & -5.598000 & -4.988500 & 0.271400 & $\mathrm{C}$ & 2.398300 & 6.212100 & -2.318000 \\
\hline $\mathrm{C}$ & -5.330300 & -5.626900 & 1.648300 & $\mathrm{H}$ & 3.278100 & 6.225000 & -2.964500 \\
\hline $\mathrm{H}$ & -4.289500 & -5.964700 & 1.724200 & $\mathrm{C}$ & 1.490800 & 7.257300 & -2.376600 \\
\hline $\mathrm{H}$ & -5.973700 & -6.498100 & 1.815500 & $\mathrm{H}$ & 1.644800 & 8.084100 & -3.066300 \\
\hline $\mathrm{H}$ & -5.509200 & -4.924300 & 2.468200 & $\mathrm{C}$ & 0.367600 & 7.255200 & -1.527600 \\
\hline $\mathrm{C}$ & -5.503800 & -6.072100 & -0.820400 & $\mathrm{H}$ & -0.333200 & 8.087700 & -1.561700 \\
\hline $\mathrm{H}$ & -5.628400 & -5.652000 & -1.823700 & $\mathrm{C}$ & 0.164000 & 6.207800 & -0.645700 \\
\hline $\mathrm{H}$ & -6.283800 & -6.830900 & -0.685000 & $\mathrm{H}$ & -0.686800 & 6.243500 & 0.032700 \\
\hline $\mathrm{H}$ & -4.534500 & -6.582400 & -0.791100 & $\mathrm{C}$ & 1.065700 & 5.114500 & -0.588000 \\
\hline $\mathrm{C}$ & -9.455300 & -2.259700 & -1.560400 & $\mathrm{C}$ & 0.907600 & 4.023500 & 0.346100 \\
\hline $\mathrm{C}$ & -8.823600 & -0.886800 & -1.535300 & $\mathrm{C}$ & 3.335000 & 2.071400 & 2.465900 \\
\hline $\mathrm{C}$ & -8.637400 & 0.043100 & -2.562700 & $\mathrm{C}$ & 4.512500 & 2.831100 & 2.545400 \\
\hline $\mathrm{H}$ & -8.891700 & -0.086600 & -3.594100 & $\mathrm{H}$ & 4.505200 & 3.888400 & 2.272100 \\
\hline $\mathrm{C}$ & -8.051200 & 1.208700 & -1.996300 & $\mathrm{C}$ & 5.700300 & 2.206400 & 2.934500 \\
\hline $\mathrm{H}$ & -7.776800 & 2.097400 & -2.531100 & $\mathrm{H}$ & 6.626000 & 2.784100 & 2.961500 \\
\hline $\mathrm{C}$ & -7.883100 & 0.982500 & -0.628100 & $\mathrm{C}$ & 5.708400 & 0.844400 & 3.265000 \\
\hline $\mathrm{C}$ & -7.367000 & 1.869800 & 0.469700 & $\mathrm{C}$ & 4.511400 & 0.110700 & 3.240400 \\
\hline $\mathrm{C}$ & -6.804400 & 3.203500 & -0.071800 & $\mathrm{H}$ & 4.508400 & -0.951900 & 3.522500 \\
\hline $\mathrm{C}$ & -5.335900 & 4.135800 & -1.766700 & $\mathrm{C}$ & 3.325400 & 0.712400 & 2.831900 \\
\hline $\mathrm{H}$ & -5.903900 & 5.070400 & -1.595300 & $\mathrm{H}$ & 2.400400 & 0.130200 & 2.766800 \\
\hline $\mathrm{H}$ & -5.308700 & 3.898500 & -2.845900 & $\mathrm{C}$ & 6.992600 & 0.139000 & 3.593200 \\
\hline $\mathrm{C}$ & -3.988600 & 4.130700 & -1.104300 & $\mathrm{H}$ & 7.879800 & 0.802100 & 3.607400 \\
\hline $\mathrm{C}$ & -3.822300 & 4.752800 & 0.142900 & $\mathrm{H}$ & 6.944300 & -0.447000 & 4.529600 \\
\hline $\mathrm{H}$ & -4.663100 & 5.287600 & 0.598300 & $\mathrm{C}$ & 8.160300 & -0.738500 & 1.633700 \\
\hline $\mathrm{C}$ & -2.604600 & 4.665000 & 0.817500 & $\mathrm{C}$ & 8.102600 & -1.842800 & 0.550300 \\
\hline $\mathrm{H}$ & -2.474000 & 5.148500 & 1.787400 & $\mathrm{C}$ & 7.822500 & -1.148700 & -0.757500 \\
\hline $\mathrm{C}$ & -1.556500 & 3.929600 & 0.240600 & $\mathrm{C}$ & 8.346100 & 0.051400 & -1.251000 \\
\hline $\mathrm{C}$ & -1.695200 & 3.348800 & -1.032200 & H & 9.023500 & 0.704900 & -0.732100 \\
\hline
\end{tabular}




\begin{tabular}{|c|c|c|c|c|c|c|c|}
\hline $\mathrm{C}$ & 7.817500 & 0.252000 & -2.554200 & $\mathrm{C}$ & 3.775800 & -3.512800 & 3.488700 \\
\hline $\mathrm{H}$ & 8.035300 & 1.082900 & -3.192900 & $\mathrm{H}$ & 4.518100 & -4.125200 & 4.014600 \\
\hline $\mathrm{C}$ & 6.975200 & -0.827100 & -2.847500 & $\mathrm{H}$ & 2.813200 & -3.655500 & 3.992200 \\
\hline $\mathrm{C}$ & 6.192700 & -1.140000 & -4.102700 & $\mathrm{H}$ & 4.064000 & -2.457800 & 3.617500 \\
\hline $\mathrm{C}$ & 4.874700 & -1.783700 & -3.740300 & $\mathrm{C}$ & -9.381100 & -2.850300 & -2.981800 \\
\hline $\mathrm{C}$ & 4.366500 & -3.046600 & -4.054200 & $\mathrm{H}$ & -10.029000 & -2.302100 & -3.675500 \\
\hline $\mathrm{H}$ & 4.850200 & -3.805700 & -4.634000 & $\mathrm{H}$ & -9.706900 & -3.897500 & -2.986000 \\
\hline $\mathrm{C}$ & 3.071800 & -3.153300 & -3.474800 & $\mathrm{H}$ & -8.366300 & -2.814600 & -3.390000 \\
\hline $\mathrm{H}$ & 2.424900 & -4.002800 & -3.552500 & $\mathrm{C}$ & -10.931500 & -2.116900 & -1.141900 \\
\hline $\mathrm{C}$ & 2.795300 & -1.953600 & -2.810500 & $\mathrm{H}$ & -11.029200 & -1.716700 & -0.127100 \\
\hline $\mathrm{C}$ & 1.586500 & -1.569200 & -1.986800 & $\mathrm{H}$ & -11.448400 & -3.082700 & -1.172400 \\
\hline $\mathrm{C}$ & 1.830800 & -1.922300 & -0.536700 & $\mathrm{H}$ & -11.463900 & -1.431900 & -1.812800 \\
\hline $\mathrm{C}$ & 1.150500 & -1.501400 & 0.608800 & $\mathrm{C}$ & -8.527000 & 2.229600 & 1.414700 \\
\hline $\mathrm{H}$ & 0.321600 & -0.815500 & 0.639900 & $\mathrm{H}$ & -8.853400 & 1.363700 & 2.000300 \\
\hline $\mathrm{C}$ & 1.736100 & -2.154100 & 1.730900 & $\mathrm{H}$ & -9.394700 & 2.598400 & 0.851500 \\
\hline $\mathrm{H}$ & 1.417800 & -2.049400 & 2.747000 & $\mathrm{H}$ & -8.239000 & 3.023300 & 2.120300 \\
\hline $\mathrm{C}$ & 2.773700 & -2.964600 & 1.262300 & $\mathrm{C}$ & -2.154300 & 0.177900 & 0.227100 \\
\hline $\mathrm{C}$ & 3.694000 & -3.907000 & 2.000600 & $\mathrm{H}$ & -2.585200 & 0.320200 & -0.767700 \\
\hline $\mathrm{C}$ & 5.087700 & -3.850600 & 1.414800 & $\mathrm{H}$ & -1.155200 & -0.273900 & 0.101100 \\
\hline $\mathrm{C}$ & 5.913300 & -4.869300 & 0.926500 & $\mathrm{H}$ & -2.000600 & 1.169500 & 0.672600 \\
\hline $\mathrm{H}$ & 5.668100 & -5.908800 & 0.850000 & $\mathrm{C}$ & -2.249600 & -1.085000 & 2.394300 \\
\hline $\mathrm{C}$ & 7.154000 & -4.285800 & 0.552000 & $\mathrm{H}$ & -1.878000 & -0.195400 & 2.919700 \\
\hline $\mathrm{H}$ & 7.993800 & -4.814900 & 0.150000 & $\mathrm{H}$ & -1.377500 & -1.700800 & 2.133800 \\
\hline $\mathrm{C}$ & 7.079600 & -2.912300 & 0.814500 & $\mathrm{H}$ & -2.855000 & -1.665800 & 3.096400 \\
\hline $\mathrm{C}$ & 9.515000 & -2.456200 & 0.517300 & $\mathrm{~N}$ & -5.184400 & 0.562000 & 0.558000 \\
\hline $\mathrm{H}$ & 10.284200 & -1.675000 & 0.423200 & $\mathrm{H}$ & -5.096000 & 0.480800 & -0.441700 \\
\hline $\mathrm{H}$ & 9.632600 & -3.123900 & -0.345600 & $\mathrm{~N}$ & -4.492300 & -2.770800 & 0.844600 \\
\hline $\mathrm{H}$ & 9.726100 & -3.036600 & 1.423200 & $\mathrm{H}$ & -5.109800 & -2.556100 & 1.607000 \\
\hline $\mathrm{C}$ & 7.035300 & -2.100000 & -4.964600 & $\mathrm{~N}$ & -8.362500 & -0.307400 & -0.347400 \\
\hline $\mathrm{H}$ & 8.008000 & -1.655300 & -5.207100 & $\mathrm{H}$ & -8.344000 & -0.754200 & 0.552800 \\
\hline $\mathrm{H}$ & 6.531300 & -2.333800 & -5.909100 & $\mathrm{~N}$ & -0.310500 & 3.716000 & 0.959800 \\
\hline $\mathrm{H}$ & 7.231800 & -3.044900 & -4.446600 & $\mathrm{~N}$ & 2.111500 & 2.674500 & 1.964500 \\
\hline $\mathrm{C}$ & 5.918400 & 0.153000 & -4.894000 & $\mathrm{~N}$ & 6.985400 & -1.688100 & -1.744500 \\
\hline $\mathrm{H}$ & 6.844500 & 0.580900 & -5.293900 & $\mathrm{H}$ & 6.477600 & -2.554800 & -1.669800 \\
\hline $\mathrm{H}$ & 5.443100 & 0.921300 & -4.274200 & $\mathrm{~N}$ & 3.906800 & -1.116700 & -2.973300 \\
\hline $\mathrm{H}$ & 5.253900 & -0.044500 & -5.744000 & $\mathrm{H}$ & 3.994900 & -0.182300 & -2.608100 \\
\hline $\mathrm{C}$ & 0.360300 & -2.361700 & -2.479500 & $\mathrm{~N}$ & 2.821900 & -2.826300 & -0.132300 \\
\hline $\mathrm{H}$ & 0.505700 & -3.442100 & -2.365800 & $\mathrm{H}$ & 3.466300 & -3.300300 & -0.743800 \\
\hline $\mathrm{H}$ & 0.154900 & -2.159900 & -3.535800 & $\mathrm{~N}$ & 5.798800 & -2.649300 & 1.319700 \\
\hline $\mathrm{H}$ & -0.543300 & -2.104600 & -1.900900 & $\mathrm{H}$ & 5.481600 & -1.746100 & 1.645900 \\
\hline $\mathrm{C}$ & 3.124300 & -5.332900 & 1.876400 & 0 & -6.907300 & 4.302000 & 0.418200 \\
\hline $\mathrm{H}$ & 3.102000 & -5.670300 & 0.834300 & 0 & -6.089500 & 3.000600 & -1.231900 \\
\hline $\mathrm{H}$ & 2.093800 & -5.374300 & 2.250500 & 0 & 7.157100 & -0.893100 & 2.566900 \\
\hline $\mathrm{H}$ & 3.716500 & -6.053700 & 2.450400 & 0 & 8.960800 & 0.158800 & 1.727700 \\
\hline
\end{tabular}

Cartesian coordinates of the optimized first excited-state structure of complex DPAC-bisC4P $\supset \mathbf{C 4}{ }^{2-}$ :

\begin{tabular}{|c|c|c|c|c|c|c|c|}
\hline Symbol & $\mathrm{X}$ & $Y$ & $Z$ & Symbo & $\mathrm{X}$ & $Y$ & $Z$ \\
\hline $\mathrm{C}$ & -1.180400 & 2.959600 & -1.532700 & $\mathrm{C}$ & -0.387600 & 4.038100 & 3.797200 \\
\hline $\mathrm{C}$ & -1.308300 & 1.566400 & -1.599700 & $\mathrm{H}$ & -1.273000 & 4.142700 & 4.383500 \\
\hline $\mathrm{H}$ & -1.397700 & 0.977700 & -2.488800 & $\mathrm{C}$ & 0.833700 & 4.732100 & 4.009700 \\
\hline $\mathrm{C}$ & 9.158800 & -0.585600 & 1.248800 & $\mathrm{H}$ & 1.017600 & 5.452000 & 4.775600 \\
\hline $\mathrm{H}$ & 9.041100 & 0.264000 & 1.928900 & $\mathrm{C}$ & 1.742700 & 4.309400 & 3.029900 \\
\hline $\mathrm{H}$ & 9.894700 & -0.317100 & 0.486000 & $\mathrm{~N}$ & 2.805200 & 5.767300 & 0.514700 \\
\hline $\mathrm{H}$ & 9.560500 & -1.424300 & 1.827600 & $\mathrm{C}$ & 3.184000 & 5.913900 & 1.851600 \\
\hline $\mathrm{C}$ & -1.280200 & 1.066600 & -0.271000 & $\mathrm{C}$ & 3.517600 & 7.258800 & 2.057400 \\
\hline $\mathrm{H}$ & -1.338700 & 0.036000 & 0.008900 & $\mathrm{H}$ & 3.844400 & 7.701200 & 2.970700 \\
\hline $\mathrm{C}$ & -1.132900 & 2.157300 & 0.596800 & $\mathrm{C}$ & 3.336800 & 7.944100 & 0.823100 \\
\hline $\mathrm{C}$ & -1.165800 & 2.184400 & 2.107300 & $\mathrm{H}$ & 3.506900 & 8.983600 & 0.659500 \\
\hline $\mathrm{C}$ & -0.214700 & 3.202000 & 2.686900 & $\mathrm{C}$ & 2.894100 & 7.009100 & -0.120500 \\
\hline
\end{tabular}




\begin{tabular}{|c|c|c|c|}
\hline $\mathrm{C}$ & 3.176800 & 4.744800 & 2.811700 \\
\hline$C$ & 4.023200 & 3.591700 & 2.242100 \\
\hline $\mathrm{H}$ & 3.936500 & 2.681000 & 2.851000 \\
\hline 1 & 5.079100 & 3.865300 & 2.179700 \\
\hline H & 3.708800 & 3.315100 & 1.21720 \\
\hline C & 3.782300 & 5.172500 & 4.167000 \\
\hline $\mathrm{H}$ & 3.232900 & 6.013900 & 4.600900 \\
\hline I & 4.825800 & 5.478000 & 4.045800 \\
\hline 11 & 3.748400 & 4.343300 & 4.880800 \\
\hline C & 2.550900 & 7.171000 & -1.583200 \\
\hline $\mathrm{C}$ & 1.127400 & 6.704900 & -1.802500 \\
\hline $\mathrm{C}$ & -0.082100 & 7.403600 & -1.645100 \\
\hline H & -0.187600 & 8.422800 & -1.347000 \\
\hline $\mathrm{C}$ & -1.141000 & 6.503700 & -1.930600 \\
\hline 1 & -2.186700 & 6.720900 & -1.887400 \\
\hline C & -0.564000 & 5.268600 & -2.261500 \\
\hline $\mathrm{C}$ & -1.194300 & 3.961700 & -2.654400 \\
\hline $\mathrm{C}$ & -2.668000 & 4.213200 & -3.050100 \\
\hline C & -4.856900 & 4.603300 & -2.057100 \\
\hline 1 & -5.119200 & 4.937500 & -3.080100 \\
\hline 1 & -5.127100 & 5.375400 & -1.311300 \\
\hline & -5.453500 & 3.262300 & -1.720100 \\
\hline מ & -6.759700 & 2.950500 & -2.121000 \\
\hline H & -7.354200 & 3.681300 & -2.665400 \\
\hline $\mathrm{U}$ & -7.304600 & 1.694100 & -1.840000 \\
\hline $\mathrm{H}$ & -8.319700 & 1.454700 & -2.150700 \\
\hline $\mathrm{C}$ & -6.523400 & 0.745200 & -1.165100 \\
\hline $\mathrm{C}$ & -5.223900 & 1.059700 & -0.728900 \\
\hline H & -4.622300 & 0.325000 & -0.188800 \\
\hline C & -4.698900 & 2.319500 & -1.004800 \\
\hline H & -3.677300 & 2.571300 & -0.682400 \\
\hline C & -7.030400 & -1.494600 & -2.025600 \\
\hline 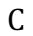 & -7.401900 & -1.070200 & -3.332900 \\
\hline 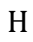 & -7.694300 & -0.034300 & -3.497800 \\
\hline $\mathrm{C}$ & -7.393400 & -1.955100 & -4.385600 \\
\hline $\mathrm{H}$ & -7.682900 & -1.630500 & -5.381600 \\
\hline C & -6.992300 & -3.303700 & -4.189200 \\
\hline H & -6.986700 & -3.981300 & -5.038900 \\
\hline C & -6.604200 & -3.741000 & -2.944400 \\
\hline $\mathrm{H}$ & -6.283000 & -4.772100 & -2.807500 \\
\hline C & -6.619000 & -2.857300 & -1.828100 \\
\hline 5 & -6.568800 & -2.497100 & 0.571800 \\
\hline 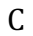 & -6.487100 & -3.041500 & 1.907100 \\
\hline C & -6.25 & -4.425100 & 2.165100 \\
\hline $\mathrm{H}$ & -6.313300 & -5.135100 & 1.341000 \\
\hline $\mathrm{C}$ & -6.107800 & -4.900600 & 3.450900 \\
\hline $\mathrm{H}$ & -5.941700 & -5.961800 & 3.623400 \\
\hline C & -6.124100 & -4.012200 & 4.542800 \\
\hline $\mathrm{H}$ & -5.934600 & -4.383200 & 5.546000 \\
\hline $\mathrm{C}$ & -6.392500 & -2.671300 & 4.324800 \\
\hline $\mathrm{H}$ & -6.433600 & -1.985000 & 5.173000 \\
\hline $\mathrm{C}$ & -6.619900 & -2.164800 & 3.023400 \\
\hline $\mathrm{C}$ & 5100 & -0.79 & 2.8 \\
\hline $\mathrm{C}$ & -7.270100 & 0.070600 & 3.925100 \\
\hline $\mathrm{H}$ & -6.991400 & -0.270700 & 4.924000 \\
\hline $\mathrm{C}$ & -7.826200 & 1.328500 & 3.762600 \\
\hline $\mathrm{H}$ & -7.969600 & 1.984700 & 4.616200 \\
\hline $\mathrm{C}$ & -8.217500 & 1.751400 & 2.478200 \\
\hline H & -8.695500 & 2.720400 & 2.353400 \\
\hline C & -7.990500 & 0.940000 & 1.380300 \\
\hline $\mathrm{H}$ & -8.330700 & 1.273200 & 0.401900 \\
\hline C & -7.344900 & -0.318200 & 1.510100 \\
\hline $\mathrm{C}$ & -6.991400 & -1.137700 & 0.374200 \\
\hline $\mathrm{C}$ & -5.023900 & -4.128700 & -0.475200 \\
\hline $\mathrm{C}$ & -5.100200 & -5.488700 & -0.807400 \\
\hline & -6.056500 & -5.943500 & -1.059500 \\
\hline
\end{tabular}

\begin{tabular}{|c|c|c|c|}
\hline C & -3.930700 & -6.254100 & -0.821800 \\
\hline $\mathrm{H}$ & -3.982900 & -7.305500 & -1.097100 \\
\hline $\mathrm{C}$ & -2.698400 & -5.670900 & -0.496600 \\
\hline$\sqrt{3}$ & -2.642100 & -4.319400 & -0.117400 \\
\hline 11 & -1.673400 & -3.875400 & 0.148300 \\
\hline $\mathrm{C}$ & -3.798400 & -3.543700 & -0.107500 \\
\hline $\mathrm{H}$ & -3.750000 & -2.489600 & 0.174100 \\
\hline $\mathrm{C}$ & -1.436900 & -6.485600 & -0.570400 \\
\hline $\mathrm{H}$ & -1.245600 & -7.056200 & 0.362000 \\
\hline $\mathrm{H}$ & -1.415600 & -7.171900 & -1.435600 \\
\hline $\mathrm{C}$ & 0.870500 & -5.788900 & -0.201000 \\
\hline $\mathrm{C}$ & 1.800900 & -4.577800 & -0.436000 \\
\hline $\mathrm{C}$ & 3.163700 & -5.102300 & -0.796000 \\
\hline $\mathrm{C}$ & 3.821900 & -6.255700 & -0.345300 \\
\hline $\mathrm{H}$ & 3.420400 & -6.981000 & 0.334500 \\
\hline $\mathrm{C}$ & 5.107300 & -6.276000 & -0.942200 \\
\hline $\mathrm{H}$ & 5.858400 & -7.018200 & -0.787900 \\
\hline $\mathrm{C}$ & 5.222600 & -5.129800 & -1.746800 \\
\hline $\mathrm{C}$ & 6.401700 & -4.655400 & -2.566500 \\
\hline $\mathrm{C}$ & 7.326000 & -3.840400 & -1.689700 \\
\hline $\mathrm{C}$ & 8.708200 & -3.948700 & -1.495200 \\
\hline $\mathrm{H}$ & 9.357900 & -4.673700 & -1.930000 \\
\hline $\mathrm{C}$ & 9.103500 & -2.913400 & -0.602100 \\
\hline $\mathrm{H}$ & 10.097100 & -2.737000 & -0.257900 \\
\hline $\mathrm{C}$ & 7.958400 & -2.183900 & -0.262200 \\
\hline $\mathrm{C}$ & 7.804400 & -0.9 & 0.606900 \\
\hline $\mathrm{C}$ & 6.805400 & -1.231700 & 1.707300 \\
\hline $\mathrm{C}$ & 6.993100 & -1.818200 & 2.965000 \\
\hline $\mathrm{H}$ & 7.913000 & -2.185100 & 3.362200 \\
\hline $\mathrm{C}$ & 5.729400 & -1.860100 & 3.617700 \\
\hline $\mathrm{H}$ & 5.543000 & -2.262300 & 4.588500 \\
\hline $\mathrm{C}$ & 4.781300 & -1.298700 & 2.753200 \\
\hline $\mathrm{C}$ & 3.296800 & -1.088200 & 2.941700 \\
\hline $\mathrm{C}$ & 2.519000 & -2.2 & 2300 \\
\hline $\mathrm{C}$ & 1.453100 & -2.968700 & 2.940100 \\
\hline $\mathrm{H}$ & 1.031100 & -2.817200 & 3.908900 \\
\hline $\mathrm{C}$ & 1.027100 & -3.940700 & 1.996900 \\
\hline $\mathrm{H}$ & 0.239700 & -4.647400 & 2.142400 \\
\hline $\mathrm{C}$ & 1.840000 & -3.810500 & 0.863200 \\
\hline $\mathrm{C}$ & 1.221500 & -3.691600 & -1.548300 \\
\hline $\mathrm{H}$ & 1.110800 & -4.241700 & -2.488800 \\
\hline $\mathrm{H}$ & 1.872200 & -2.823300 & -1.763800 \\
\hline $\mathrm{H}$ & 0.238700 & -3.291700 & -1.273600 \\
\hline $\mathrm{C}$ & 5.94 & -3.7 & -3.7 \\
\hline $\mathrm{H}$ & 5.300500 & -4.3 & -4.436700 \\
\hline $\mathrm{H}$ & 6.797200 & -3.398700 & -4.313200 \\
\hline $\mathrm{H}$ & 5.355700 & -2.907500 & -3.423400 \\
\hline $\mathrm{C}$ & 7.149600 & -5.890200 & -3.116700 \\
\hline $\mathrm{H}$ & 6.464700 & -6.537300 & -3.673000 \\
\hline $\mathrm{H}$ & 7.582100 & -6.480900 & -2.301700 \\
\hline $\mathrm{H}$ & 7.961300 & -5.590800 & -3.785100 \\
\hline $\mathrm{C}$ & 7.337400 & 0.217100 & -0.282000 \\
\hline $\mathrm{H}$ & 6.3 & -0.0 & -0.773600 \\
\hline $\mathrm{H}$ & 8.059600 & 0.41 & -1.078000 \\
\hline $\mathrm{H}$ & 7.202900 & 1.132600 & 0.300900 \\
\hline $\mathrm{C}$ & 2.831700 & 0.199800 & 2.237200 \\
\hline $\mathrm{H}$ & 3.366200 & 1.084800 & 2.612900 \\
\hline $\mathrm{H}$ & 1.753000 & 0.372600 & 2.394400 \\
\hline $\mathrm{H}$ & 2.981400 & 0.163300 & 1.141500 \\
\hline $\mathrm{C}$ & 3.005400 & -0.956400 & 4.452700 \\
\hline $\mathrm{H}$ & 1.958400 & -0.692300 & 4.631100 \\
\hline $\mathrm{H}$ & 3.628900 & -0.173400 & 4.897100 \\
\hline $\mathrm{H}$ & 3.214100 & -1.894200 & 4.977900 \\
\hline $\mathrm{C}$ & 2.661200 & 8.653500 & -1.997400 \\
\hline $\mathrm{H}$ & 2.340100 & 8.789000 & -3.034500 \\
\hline 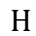 & 3.691700 & 9.007900 & -1.905300 \\
\hline
\end{tabular}




\begin{tabular}{lrrrrrrr}
$\mathrm{H}$ & 2.029200 & 9.285700 & -1.364300 & $\mathrm{~N}$ & 2.749000 & -2.774500 & 1.095300 \\
$\mathrm{C}$ & 3.538600 & 6.340100 & -2.427800 & $\mathrm{O}$ & -3.199100 & 4.157200 & -4.132300 \\
$\mathrm{H}$ & 3.462100 & 5.257700 & -2.214000 & $\mathrm{O}$ & -3.414500 & 4.540200 & -1.924000 \\
$\mathrm{H}$ & 4.572400 & 6.626700 & -2.217500 & $\mathrm{O}$ & -0.362800 & -5.531500 & -0.792100 \\
$\mathrm{H}$ & 3.353000 & 6.461200 & -3.498000 & $\mathrm{O}$ & 1.034400 & -6.829300 & 0.388100 \\
$\mathrm{C}$ & -0.485800 & 3.376900 & -3.886500 & $\mathrm{H}$ & 1.515600 & 4.616600 & -2.330300 \\
$\mathrm{H}$ & 0.539400 & 3.041000 & -3.616100 & $\mathrm{H}$ & 2.517700 & 4.874000 & 0.064600 \\
$\mathrm{H}$ & -0.387900 & 4.119900 & -4.684500 & $\mathrm{H}$ & 1.491500 & 2.936700 & 1.371900 \\
$\mathrm{H}$ & -1.011500 & 2.508300 & -4.294200 & $\mathrm{H}$ & -0.895600 & 4.250300 & 0.157500 \\
$\mathrm{C}$ & -2.616600 & 2.522900 & 2.522200 & $\mathrm{H}$ & 5.002500 & -0.541300 & 0.739700 \\
$\mathrm{H}$ & -2.884100 & 3.541200 & 2.220900 & $\mathrm{H}$ & 5.896000 & -2.434700 & -0.863300 \\
$\mathrm{H}$ & -2.745400 & 2.448500 & 3.605800 & $\mathrm{H}$ & 3.827700 & -3.498600 & -2.119000 \\
$\mathrm{H}$ & -3.327500 & 1.835500 & 2.052100 & $\mathrm{H}$ & 3.460700 & -2.448100 & 0.428500 \\
$\mathrm{C}$ & -0.779600 & 0.802200 & 2.669500 & $\mathrm{O}$ & 4.372800 & -1.289900 & -0.816400 \\
$\mathrm{H}$ & -1.468700 & 0.018600 & 2.344000 & $\mathrm{O}$ & 3.547300 & -1.882900 & -2.767100 \\
$\mathrm{H}$ & -0.769800 & 0.814200 & 3.764000 & $\mathrm{C}$ & 2.374700 & 2.536300 & -1.363800 \\
$\mathrm{H}$ & 0.235200 & 0.506900 & 2.340600 & $\mathrm{O}$ & 2.291200 & 3.152800 & -0.261400 \\
$\mathrm{~N}$ & -1.072800 & 3.316500 & -0.182400 & $\mathrm{O}$ & 2.351600 & 3.138000 & -2.467300 \\
$\mathrm{~N}$ & 1.095300 & 3.370400 & 2.222600 & $\mathrm{C}$ & 3.830900 & -0.995600 & -1.926900 \\
$\mathrm{~N}$ & 0.823600 & 5.402000 & -2.183000 & $\mathrm{C}$ & 2.477000 & 1.019100 & -1.301100 \\
$\mathrm{~N}$ & -7.015500 & -0.611400 & -0.929900 & $\mathrm{H}$ & 1.478900 & 0.597700 & -1.542100 \\
$\mathrm{~N}$ & -6.203400 & -3.267800 & -0.547100 & $\mathrm{H}$ & 2.693600 & 0.707500 & -0.250400 \\
$\mathrm{~N}$ & 4.028000 & -4.419500 & -1.659000 & $\mathrm{C}$ & 3.540400 & 0.464400 & -2.242300 \\
$\mathrm{~N}$ & 6.871100 & -2.757400 & -0.930400 & $\mathrm{H}$ & 4.475800 & 1.050000 & -2.157200 \\
$\mathrm{~N}$ & 5.451200 & -0.906300 & 1.590200 & $\mathrm{H}$ & 3.209100 & 0.570300 & -3.295000 \\
& & & & & & & \\
\hline
\end{tabular}




\section{Crystal structures of DPAC-bis/monoCHO and DPAC-bisC4P}

Table S2. CCDC numbers, crystal data, and structure refinement details for DPAC-monoCHO, DPAC-bisCHO, and DPAC-bisC4P.

\begin{tabular}{|c|c|c|c|}
\hline Crystal Data & DPAC-monoCHO & DPAC-bisCHO & DPAC-bisC4P \\
\hline CCDC No. & 1423375 & 1411071 & 1854421 \\
\hline empirical formula & $\mathrm{C} 33 \mathrm{H} 22 \mathrm{~N} 2 \mathrm{O}$ & $\mathrm{C} 34 \mathrm{H} 22 \mathrm{~N} 2 \mathrm{O} 2$ & $\begin{array}{l}\mathrm{C} 90 \mathrm{H} 90 \mathrm{~N} 10 \mathrm{O} 4, \\
\mathrm{H} 2 \mathrm{O}, 3(\mathrm{C} 2 \mathrm{H} 6 \mathrm{O})\end{array}$ \\
\hline formula wt & 462.52 & 490.53 & 1531.93 \\
\hline$T, \mathrm{~K}$ & $296(2)$ & 140 & $100(2)$ \\
\hline crystal system & Triclinic & Monoclinic & Triclinic \\
\hline space group & P-1 & P $121 / n 1$ & P-1 \\
\hline$a, \AA$ & $10.040(3)$ & $9.587(2)$ & $10.2435(6)$ \\
\hline$b, \AA$ & $15.297(4)$ & $15.465(3)$ & $20.2635(12)$ \\
\hline$c, \AA$ & $16.237(5)$ & 17.164(4) & $21.1965(11)$ \\
\hline$\alpha, \operatorname{deg}$ & $105.151(5)$ & 90 & $107.827(5)$ \\
\hline$\beta, \operatorname{deg}$ & $98.504(6)$ & $102.394(4)$ & $90.675(5)$ \\
\hline$\gamma, \operatorname{deg}$ & $96.468(5)$ & 90 & $97.043(5)$ \\
\hline$V, \AA^{3}$ & $2350.4(12)$ & $2485.3(10)$ & $4151.2(4)$ \\
\hline$Z$ & 4 & 4 & 2 \\
\hline density, $\mathrm{g} / \mathrm{cm}^{3}$ & 1.307 & 1.311 & 1.226 \\
\hline$\mu(\mathrm{Mo} \mathrm{K} \alpha), \mathrm{mm}^{-1}$ & 0.079 & 0.082 & 0.620 \\
\hline$\theta$ range, deg & $1.629-25.498$ & $1.792-27.572$ & $3.641-70.651$ \\
\hline No. of reflns collected & 16464 & 20632 & 16734 \\
\hline No. of independent reflns & 8739 & 5739 & 7691 \\
\hline$R$ (int) & 0.0492 & 0.1244 & 0.1039 \\
\hline GOF & 0.951 & 0.867 & 1.026 \\
\hline$R 1[I>2 \sigma(I)]$ & 0.0679 & 0.0605 & 0.0980 \\
\hline$w R 2[I>2 \sigma(I)]$ & 0.1661 & 0.1102 & 0.2445 \\
\hline$R 1$ (all data) & 0.1695 & 0.1580 & 0.1822 \\
\hline$w R 2$ (all data) & 0.2225 & 0.1341 & 0.3215 \\
\hline
\end{tabular}




\section{X-ray experimental}

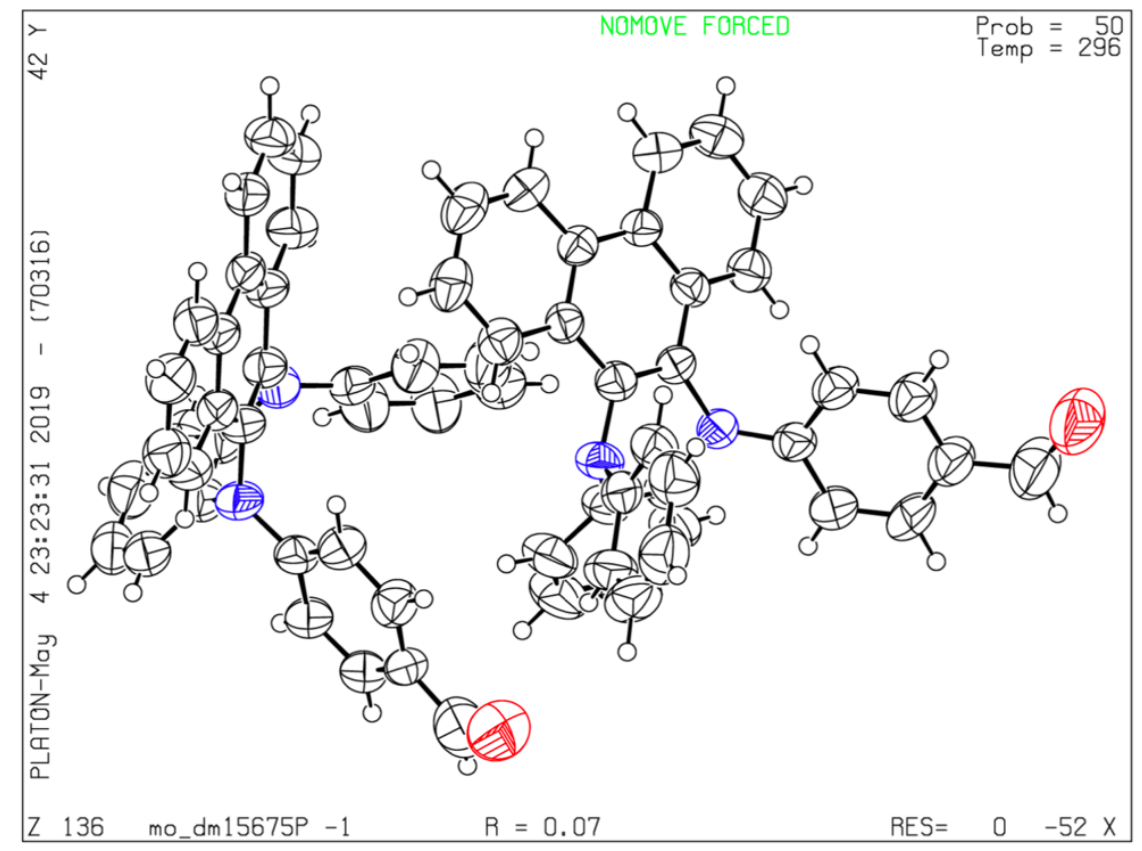

Figure S34. View of DPAC-monoCHO. Displacement ellipsoids are scaled to the 50\% probability level.

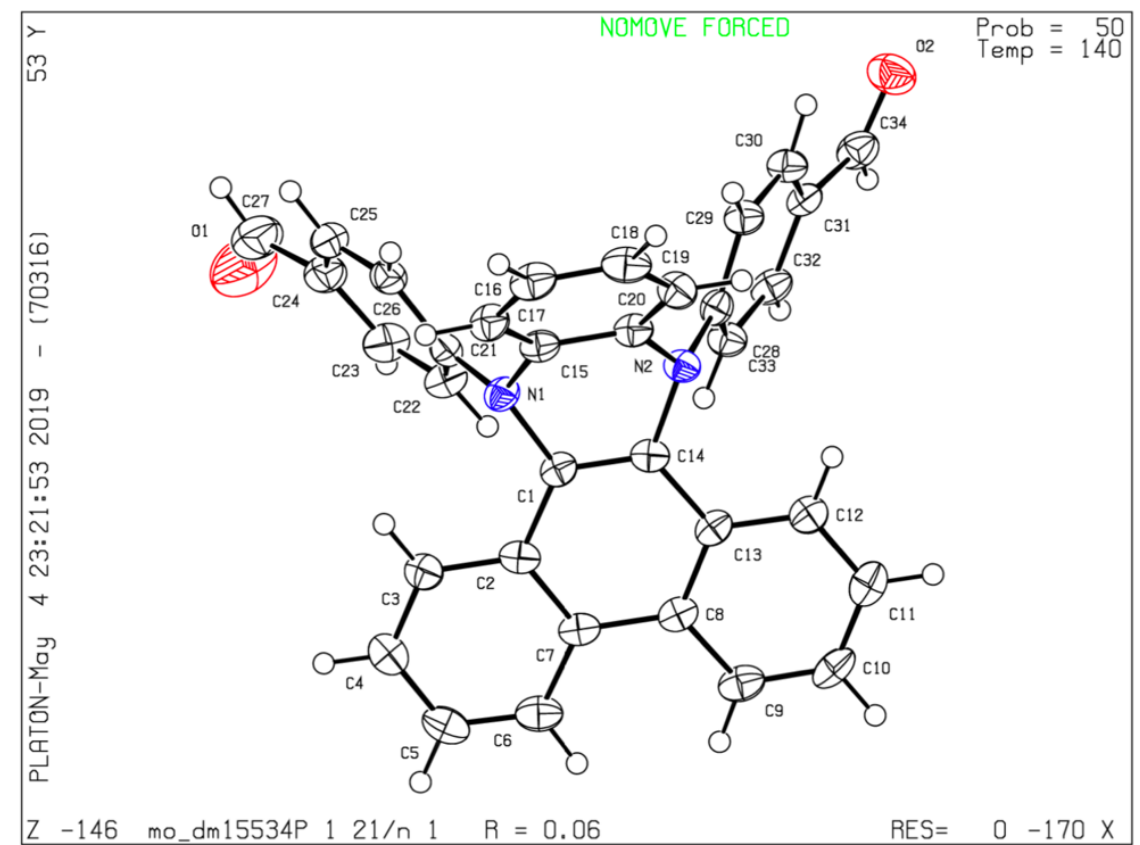

Figure S35. View of DPAC-bisCHO. Displacement ellipsoids are scaled to the 50\% probability level. 


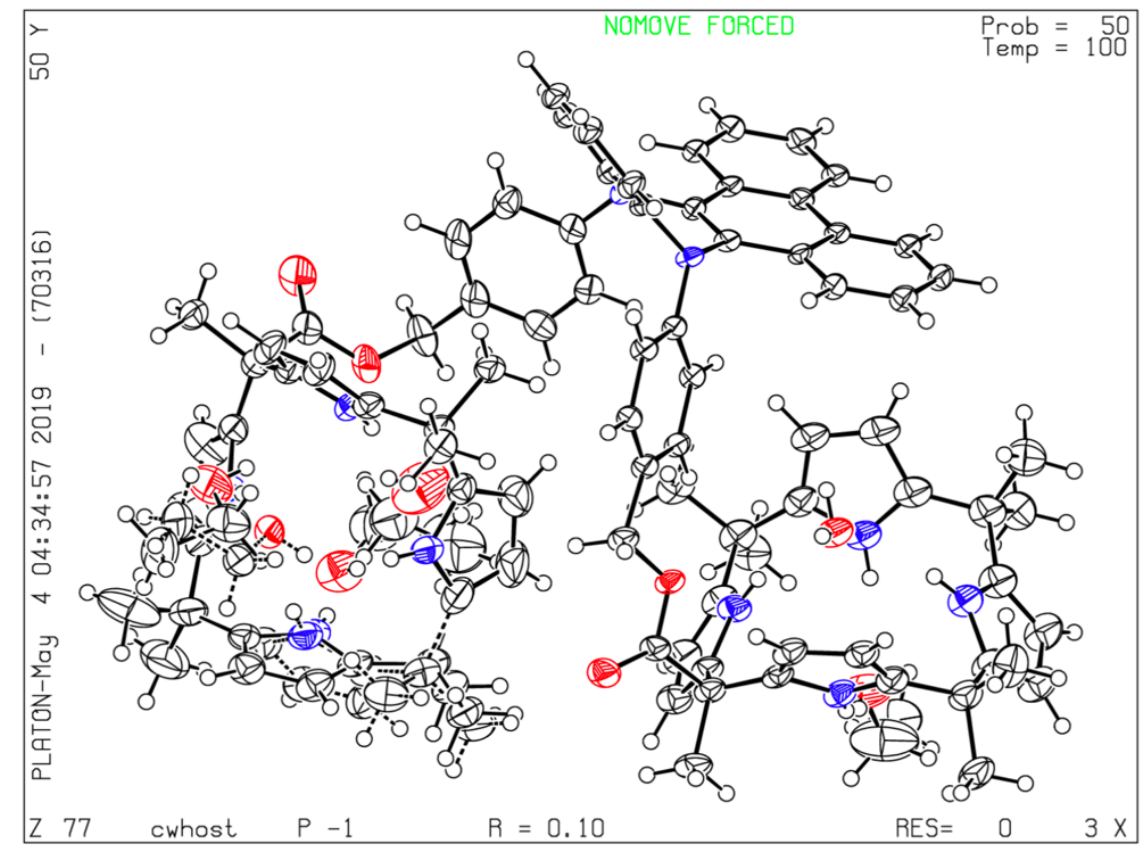

Figure S36. View of DPAC-bisC4P. Displacement ellipsoids are scaled to the 50\% probability level. 


\section{0. ${ }^{1} \mathrm{H}$ NMR, ${ }^{13} \mathrm{C}$ NMR, and HRMS spectra}

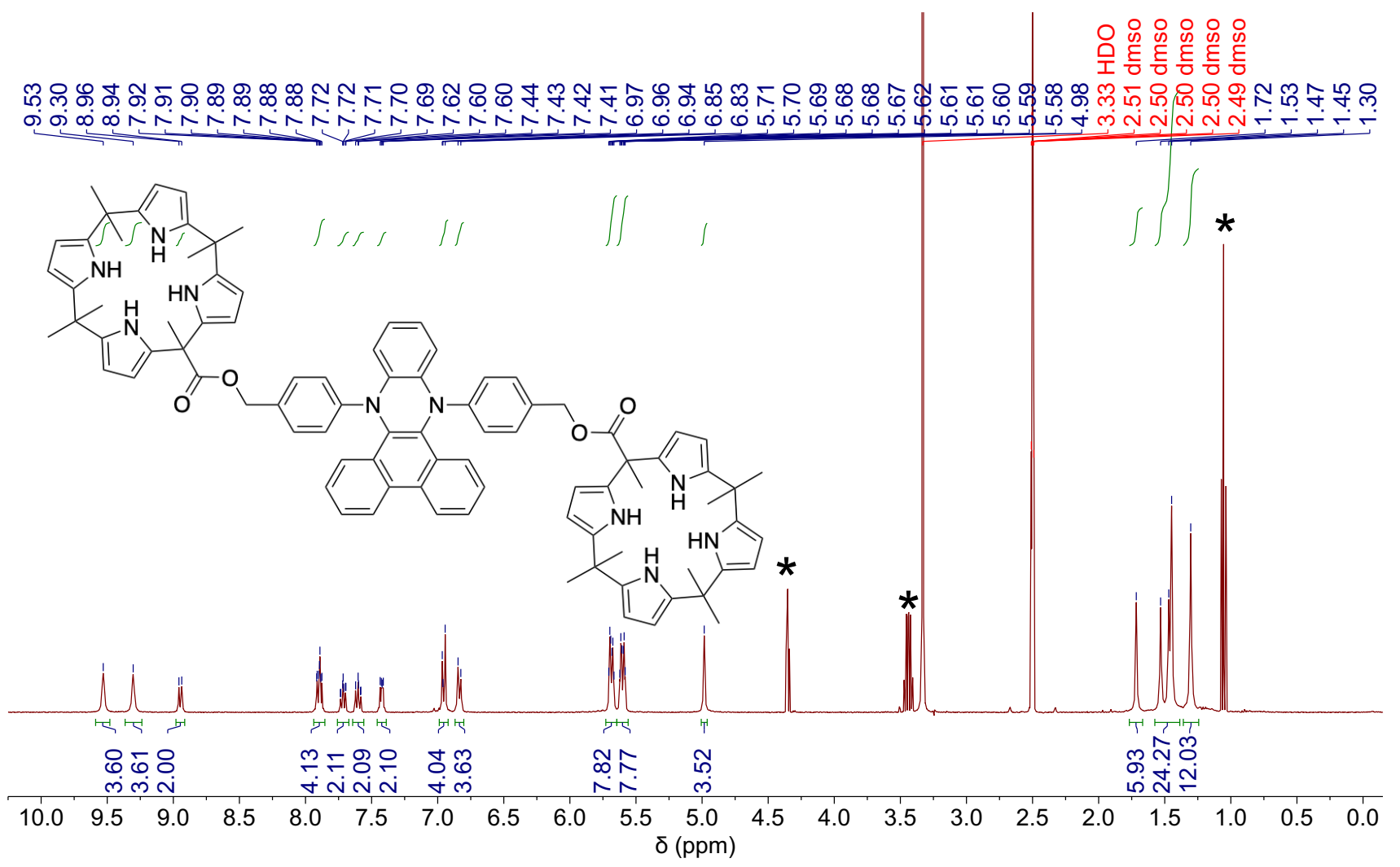

Figure S37. ${ }^{1} \mathrm{H}$ NMR spectrum of DPAC-bisC4P (DMSO- $d 6$ ). An asterisk designates residual ethanol solvent peaks.

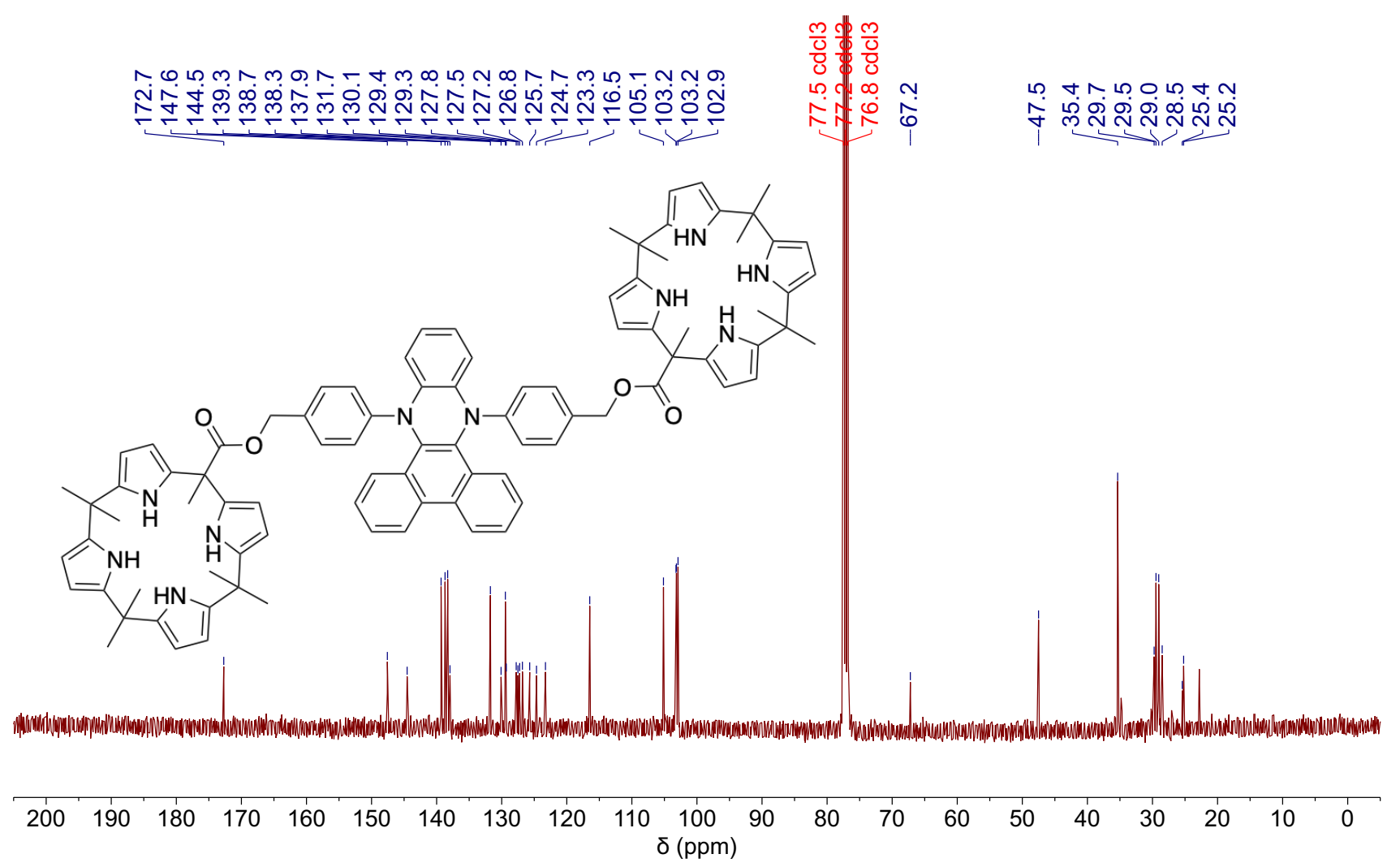

Figure S38. ${ }^{13} \mathrm{C}$ NMR spectrum of DPAC-bisC4P recorded in $\mathrm{CDCl}_{3}$. 


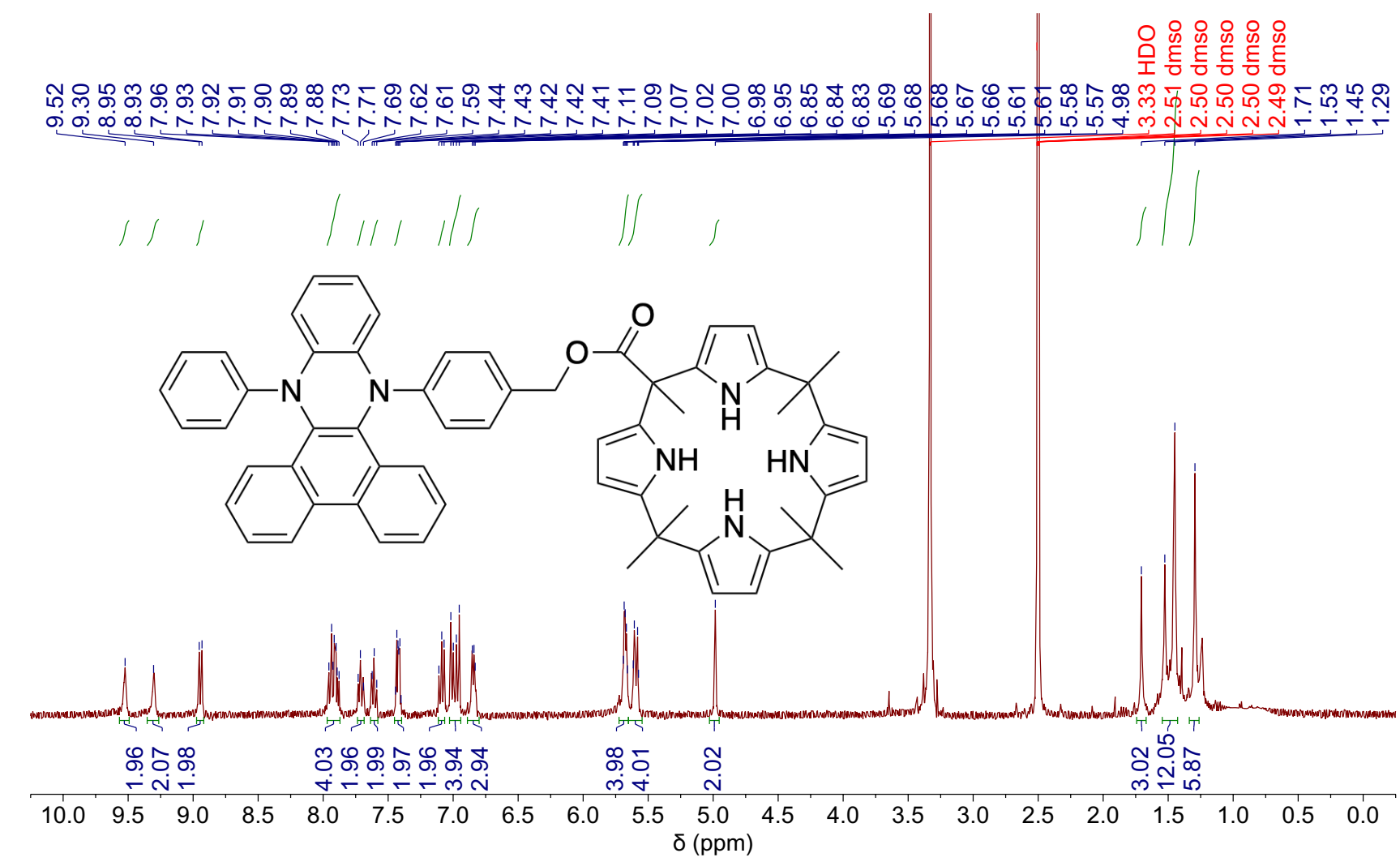

Figure S39. ${ }^{1} \mathrm{H}$ NMR spectrum of DPAC-monoC4P recorded in DMSO- $d_{6}$.
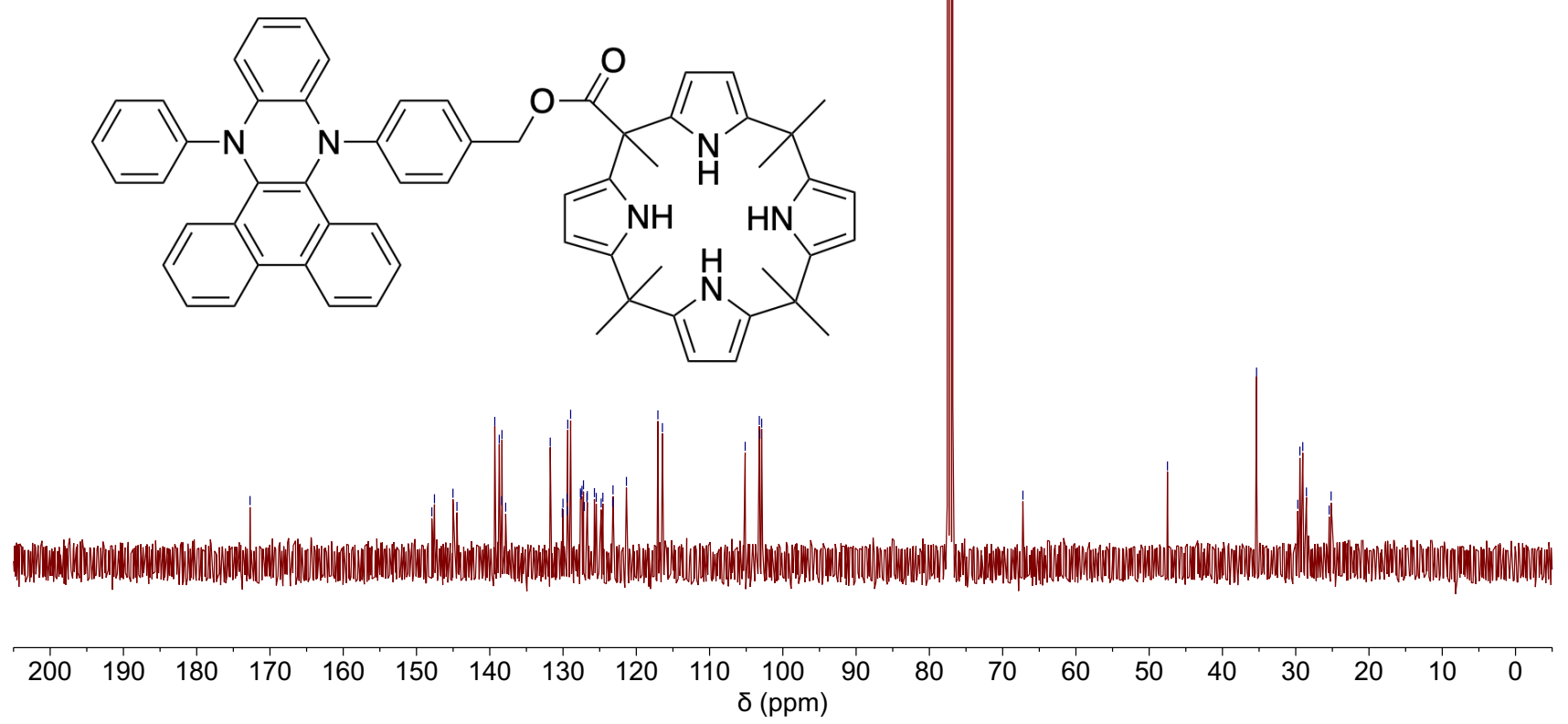

Figure S40. ${ }^{13} \mathrm{C}$ NMR spectrum of DPAC-monoC4P recorded in $\mathrm{CDCl}_{3}$. 


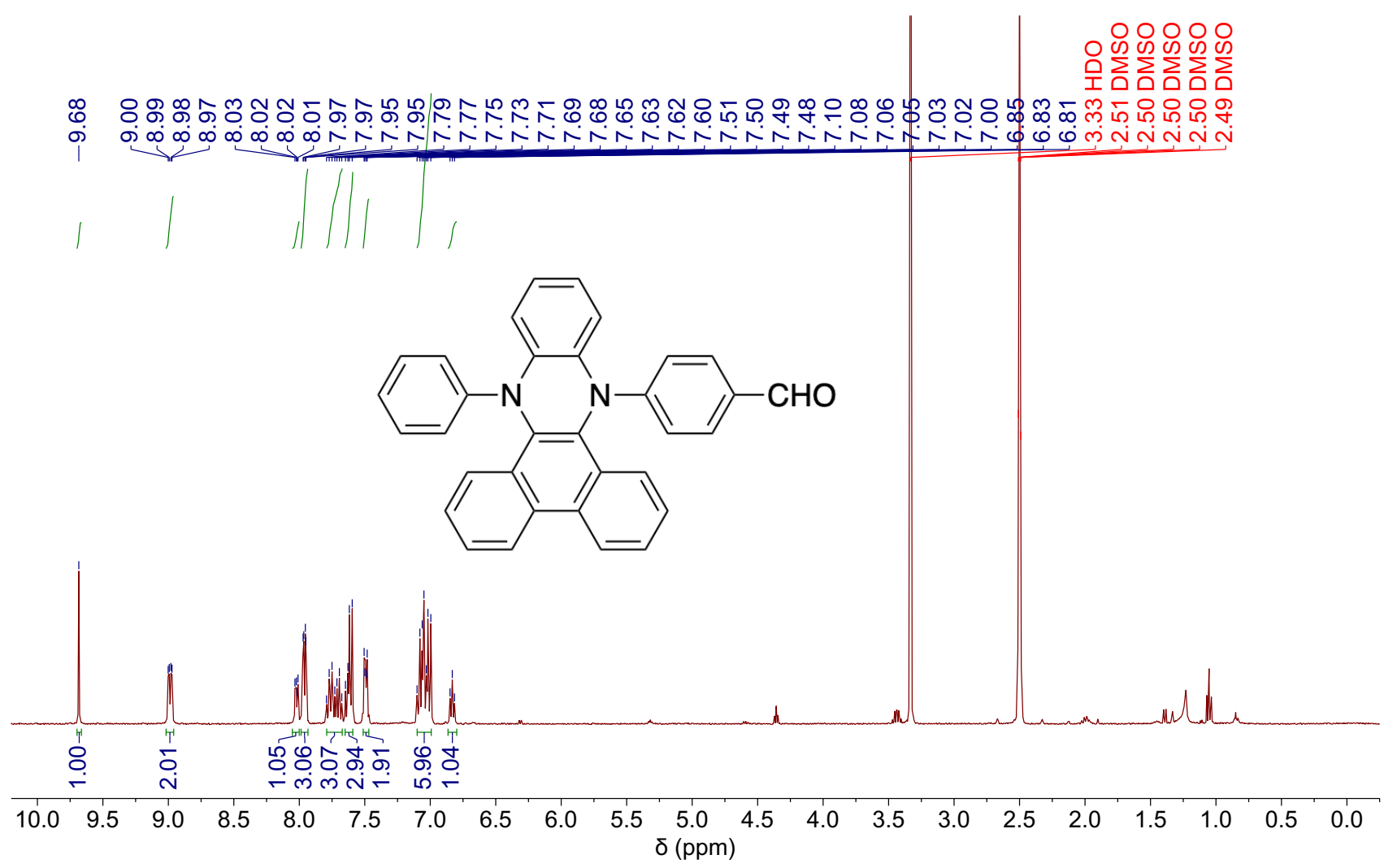

Figure S41. ${ }^{1} \mathrm{H}$ NMR spectrum of DPAC-monoCHO recorded in DMSO- $d_{6}$.

Figure S42. ${ }^{13} \mathrm{C}$ NMR spectrum of DPAC-monoCHO recorded in $\mathrm{CDCl}_{3}$. 


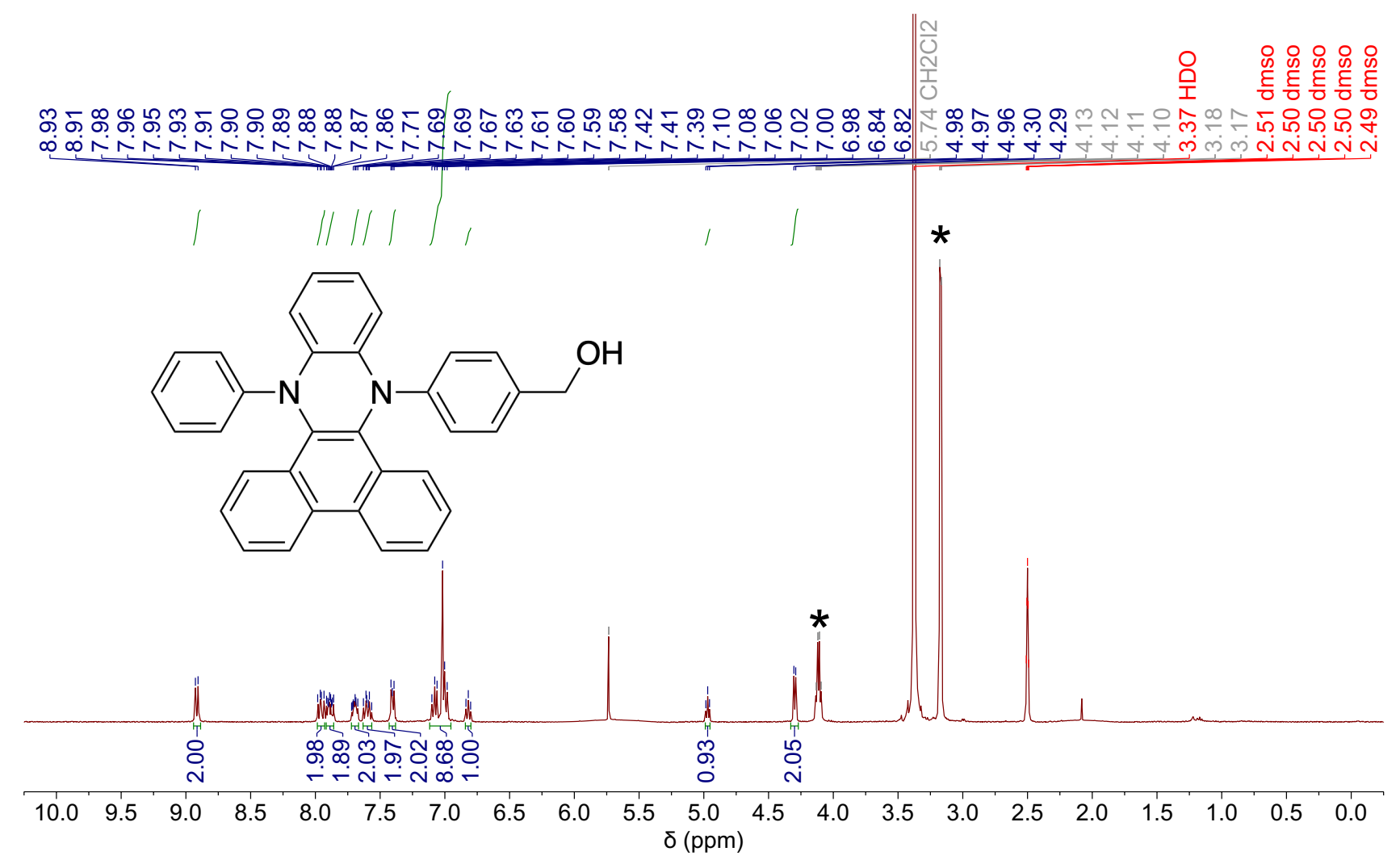

Figure S43. ${ }^{1} \mathrm{H}$ NMR spectrum of DPAC-monoOH (DMSO- $d_{6}$ ). An asterisk designates residual methanol solvent peaks.
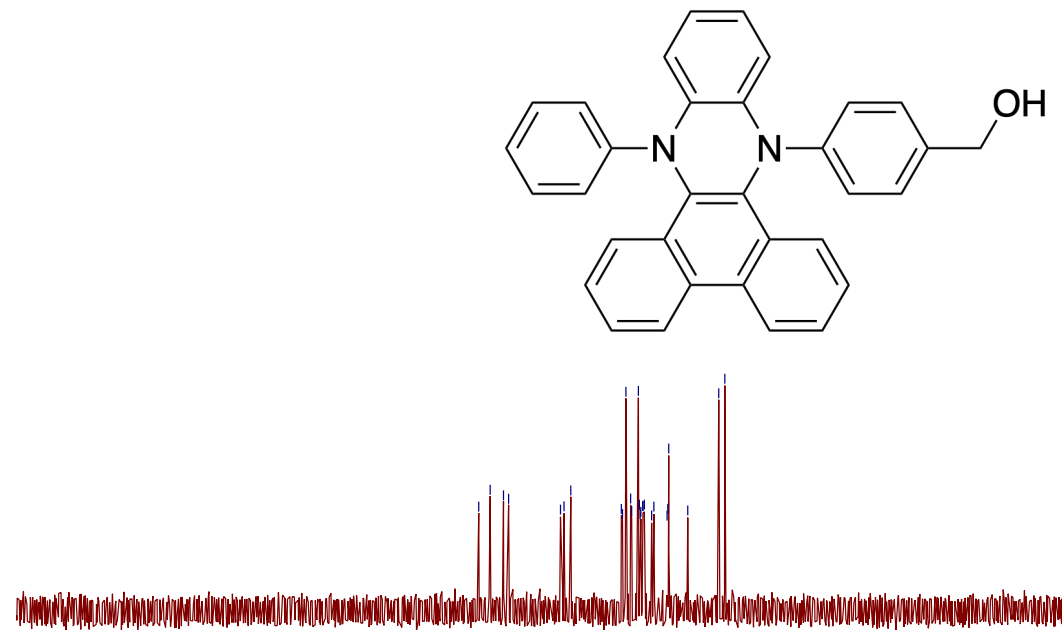

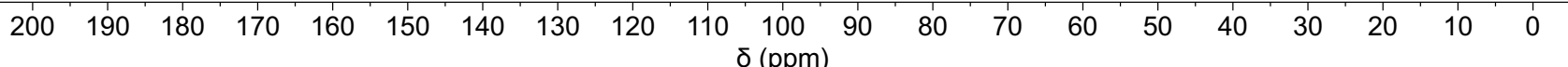

Figure S44. ${ }^{13} \mathrm{C}$ NMR spectrum of DPAC-monoOH recorded in DMSO- $d_{6}$. 
Target Compound Screening Report

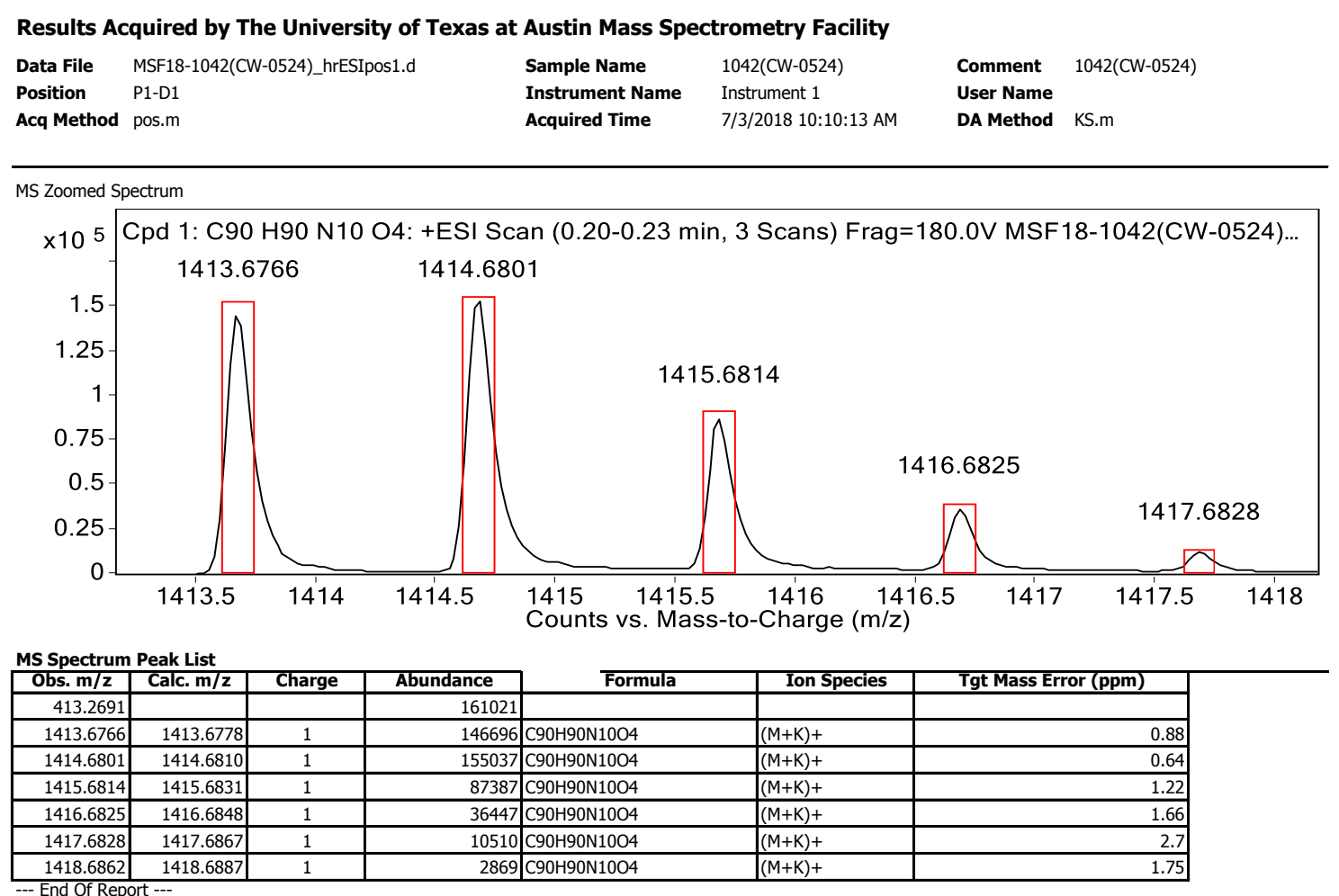

Figure S45. HR-ESI ${ }^{+}$-MS reports for [DPAC-bisC4P+K $]^{+}$.

Target Compound Screening Report

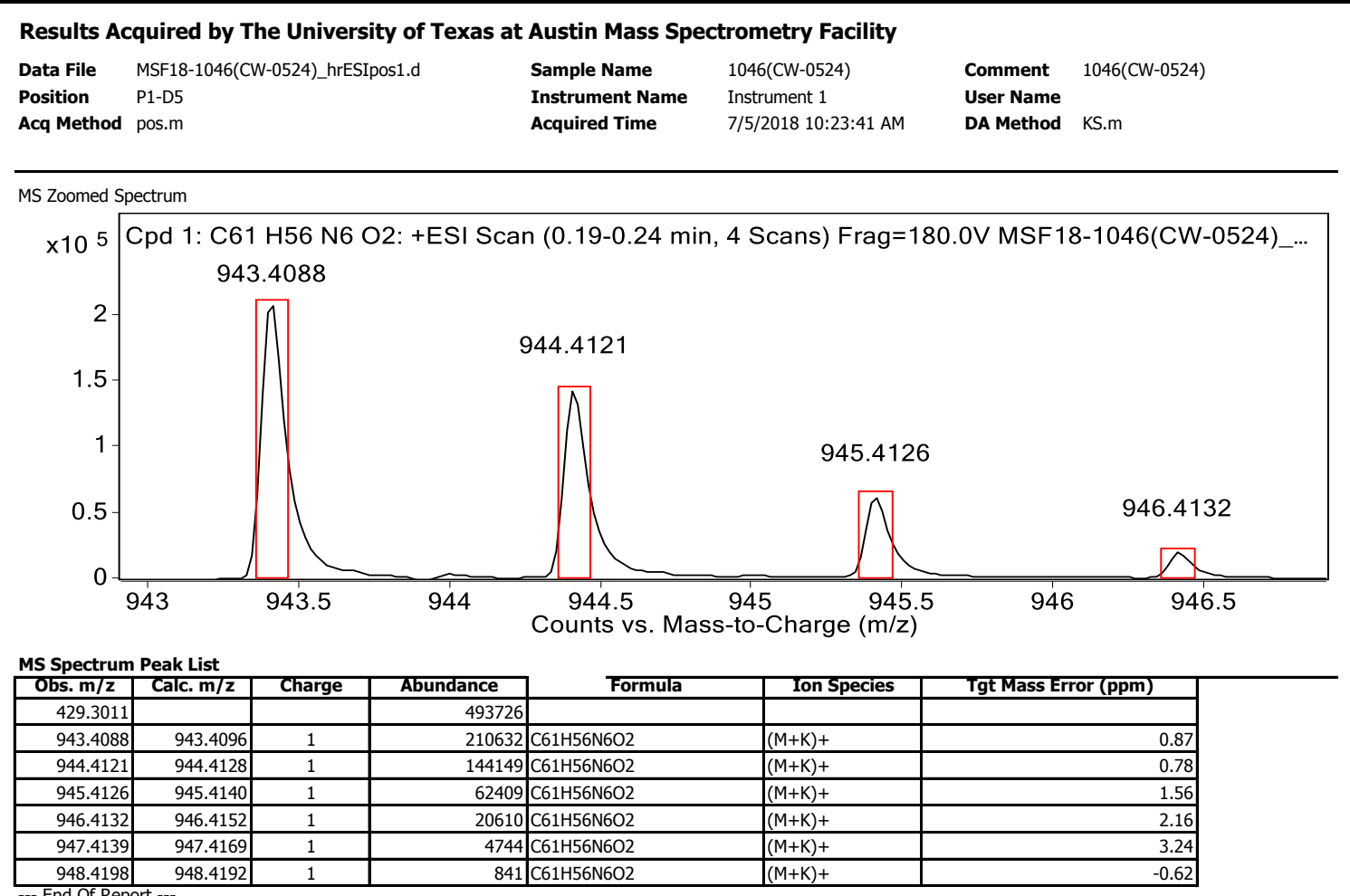

Figure S46. HR-ESI ${ }^{+}$-MS reports for $[\text {DPAC-monoC4P+K }]^{+}$. 


\section{Single Mass Analysis}

Tolerance $=50.0$ PPM / DBE: $\min =-1.5, \max =100.0$

Element prediction: Off

Number of isotope peaks used for i-FIT $=2$

Monoisotopic Mass, Even Electron lons

191 formula(e) evaluated with 10 results within limits (up to 1 closest results for each mass)

Elements Used:

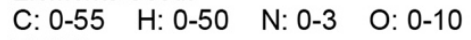

TIAN-H

ECUST institute of Fine Chem

TH-CW-5281 $8(0.324)$ Cm (5:8)

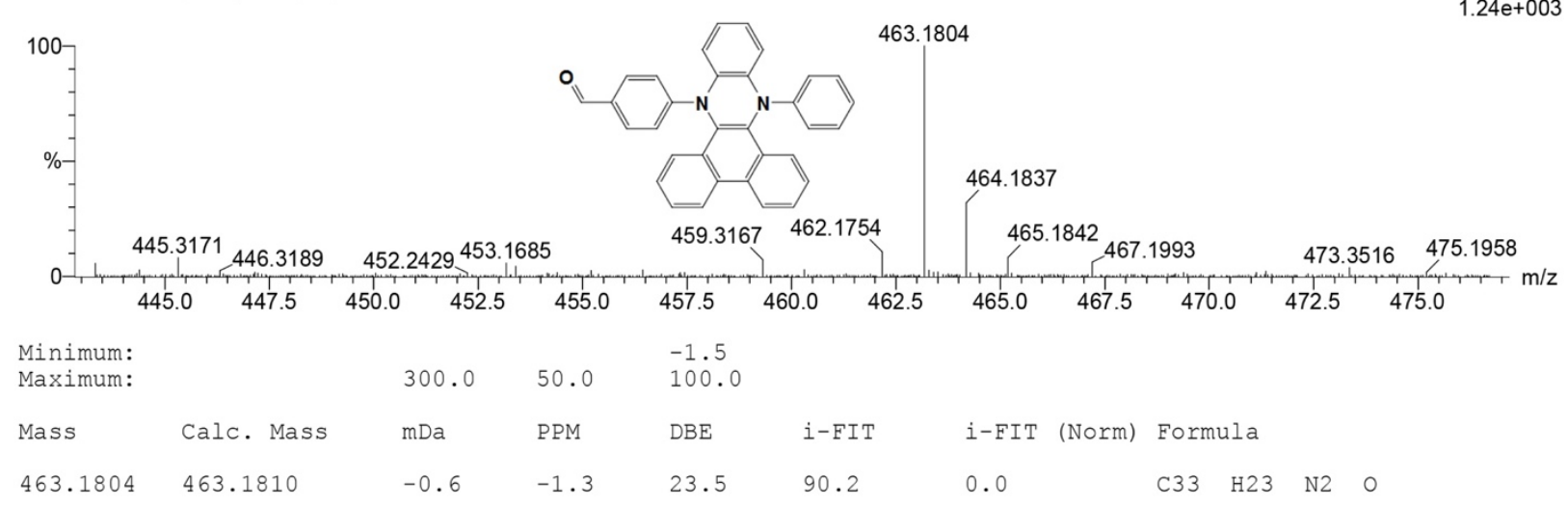

Figure S47. HR-ESI ${ }^{+}$-MS reports for [DPAC-monoCHO+H] ${ }^{+}$.

\section{Target Compound Screening Report}

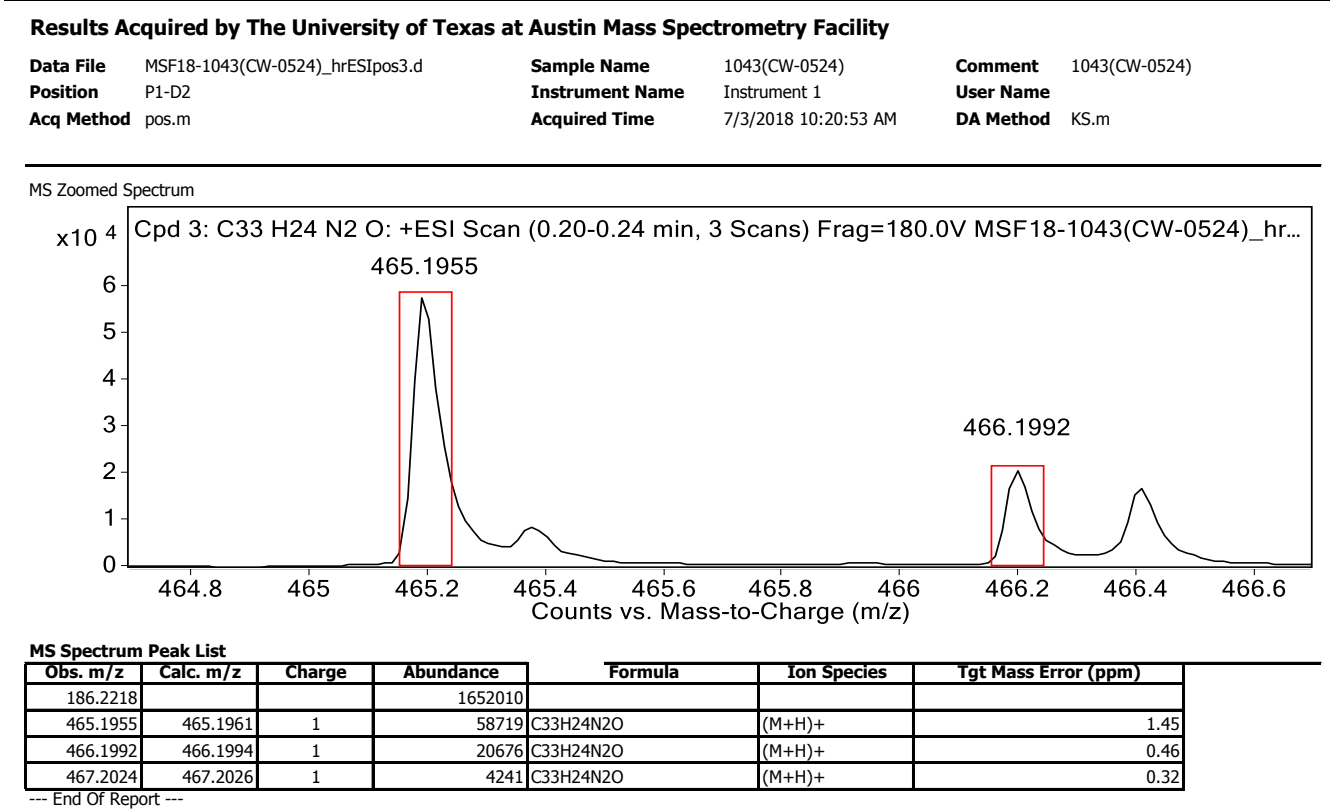

Figure S48. HR-ESI ${ }^{+}$-MS reports for $[\mathrm{DPAC}-\mathrm{monoOH}+\mathrm{H}]^{+}$. 


\section{References}

S1. Zhang, Z.; Wu, Y.-S.; Tang, K.-C.; Chen, C.-L.; Ho, J.-W.; Su, J.; Tian, H.; Chou, P.-T. Excited-State Conformational/Electronic Responses of Saddle-Shaped $N, N^{\prime}$-Disubstituted-Dihydrodibenzo[ $\left.a, c\right]$ phenazines: Wide-Tuning Emission from Red to Deep Blue and White Light Combination. J. Am. Chem. Soc. 2015, 137, 8509-8520. S2. Hargrove, A. E.; Zhong, Z.; Sessler, J. L.; Anslyn, E. V. Algorithms for the determination of binding constants and enantiomeric excess in complex host : guest equilibria using optical measurements. New J. Chem. 2010, 34, 348-354.

S3. (a) Shao, Y.; Molnar, L. F.; Jung, Y.; Kussmann, J.; Ochsenfeld, C.; Brown, S. T.; Gilbert, A. T. B.; Slipchenko, L. V.; Levchenko, S. V.; O’Neill, D. P.; DiStasio Jr, R. A.; Lochan, R. C.; Wang, T.; Beran, G. J. O.; Besley, N. A.; Herbert, J. M.; Lin, C. Y.; Van Voorhis, T.; Chien, S. H.; Sodt, A.; Steele, R. P.; Rassolov, V. A.; Maslen, P. E.; Korambath, P. P.; Adamson, R. D.; Austin, B.; Baker, J.; Byrd, E. F. C.; Dachsel, H.; Doerksen, R. J.; Dreuw, A.; Dunietz, B. D.; Dutoi, A. D.; Furlani, T. R.; Gwaltney, S. R.; Heyden, A.; Hirata, S.; Hsu, C.-P.; Kedziora, G.; Khalliulin, R. Z.; Klunzinger, P.; Lee, A. M.; Lee, M. S.; Liang, W.; Lotan, I.; Nair, N.; Peters, B.; Proynov, E. I.; Pieniazek, P. A.; Rhee, Y. M.; Ritchie, J.; Rosta, E.; David Sherrill, C.; Simmonett, A. C.; Subotnik, J. E.; Lee Woodcock III, H.; Zhang, W.; Bell, A. T.; Chakraborty, A. K.; Chipman, D. M.; Keil, F. J.; Warshel, A.; Hehre, W. J.; Schaefer III, H. F.; Kong, J.; Krylov, A. I.; Gill, P. M. W.; Head-Gordon, M. Advances in methods and algorithms in a modern quantum chemistry program package. Phys. Chem. Chem. Phys. 2006, 8, 3172-3191. (b) Gaussian 16, Revision C.01, Frisch, M. J.; Trucks, G. W.; Schlegel, H. B.; Scuseria, G. E.; Robb, M. A.; Cheeseman, J. R.; Scalmani, G.; Barone, V.; Petersson, G. A.; Nakatsuji, H.; Li, X.; Caricato, M.; Marenich, A. V.; Bloino, J.; Janesko, B. G.; Gomperts, R.; Mennucci, B.; Hratchian, H. P.; Ortiz, J. V.; Izmaylov, A. F.; Sonnenberg, J. L.; Williams-Young, D.; Ding, F.; Lipparini, F.; Egidi, F.; Goings, J.; Peng, B.; Petrone, A.; Henderson, T.; Ranasinghe, D.; Zakrzewski, V. G.; Gao, J.; Rega, N.; Zheng, G.; Liang, W.; Hada, M.; Ehara, M.; Toyota, K.; Fukuda, R.; Hasegawa, J.; Ishida, M.; Nakajima, T.; Honda, Y.; Kitao, O.; Nakai, H.; Vreven, T.; Throssell, K.; Montgomery, J. A., Jr.; Peralta, J. E.; Ogliaro, F.; Bearpark, M. J.; Heyd, J. J.; Brothers, E. N.; Kudin, K. N.; Staroverov, V. N.; Keith, T. A.; Kobayashi, R.; Normand, J.; Raghavachari, K.; Rendell, A. P.; Burant, J. C.; Iyengar, S. S.; Tomasi, J.; Cossi, M.; Millam, J. M.; Klene, M.; Adamo, C.; Cammi, R.; Ochterski, J. W.; Martin, R. L.; Morokuma, K.; Farkas, O.; Foresman, J. B.; Fox, D. J. Gaussian, Inc., Wallingford CT, 2016.

S4. Aydogan, A.; Sessler, J. L.; Akar, A.; Lynch, V. Calix[4]pyrroles with Long Alkyl Chains: Synthesis, Characterization, and Anion Binding Studies. Supramol. Chem. 2008, 20, 11-21. 